\title{
THE GOPAKUMAR-VAFA FORMULA FOR SYMPLECTIC MANIFOLDS
}

\author{
ELENY-NICOLETA IONEL AND THOMAS H. PARKER
}

\begin{abstract}
The Gopakumar-Vafa conjecture predicts that the Gromov-Witten invariants of a Calabi-Yau 3-fold can be canonically expressed in terms of integer invariants called BPS numbers. Using the methods of symplectic Gromov-Witten theory, we prove that the Gopakumar-Vafa conjecture holds for any symplectic Calabi-Yau 6-manifold, and hence for Calabi-Yau 3-folds. The results extend to all symplectic 6-manifolds and to the genus zero GW invariants of semipositive manifolds.
\end{abstract}

The Gopakumar-Vafa conjecture GV] predicts that the Gromov-Witten invariants $G W_{A, g}$ of a Calabi-Yau 3-fold can be expressed in terms of some other invariants $n_{A, h}$, called BPS numbers, by a transform between their generating functions:

$$
\sum_{\substack{A \neq 0 \\ g}} G W_{A, g} t^{2 g-2} q^{A}=\sum_{\substack{A \neq 0 \\ h}} n_{A, h} \sum_{k=1}^{\infty} \frac{1}{k}\left(2 \sin \frac{k t}{2}\right)^{2 h-2} q^{k A} .
$$

The content of the conjecture is that, while the $G W_{A, g}$ are rational numbers, the BPS numbers $n_{A, h}$ are integers. (Gopakumar and Vafa also conjectured that for each $A \in H_{2}(X, \mathbb{Z})$, the coefficients of (0.1) satisfy $n_{A, h}=0$ for large $h$; we do not address this finiteness statement here.) It is natural to enlarge the context by regarding this as a conjecture about the Gromov-Witten invariants of any closed symplectic 6-manifold $X$ that satisfies the topological Calabi-Yau condition $c_{1}(X)=0$.

Formula (0.1) can be viewed as a statement about the structure of the space of solutions to the $J$ holomorphic map equation. For a generic almost complex structure $J$, each $J$-holomorphic map is the composition $f=\varphi \circ \rho$ of a multiple-cover $\rho$ and an embedding $\varphi$. The embeddings are well-behaved: they have no nontrivial automorphisms, and the moduli space of $J$-holomorphic embeddings is a manifold. But multiply-covered maps cause severe analytical problems with transversality. In the symplectic construction of the GW invariants, these problems are avoided by lifting to a cover of the moduli space and turning on a lift-dependent perturbation $\nu$ of the equation; this destroys the multiple-cover structure and only shows that the numbers $G W_{A, g}$ are rational. But it also suggests an interpretation of the GV formula: the righthand side of 0.1$)$ might be a sum over embeddings, with the sum over $k$ counting the contributions of the multiple covers of each embedding.

This viewpoint is very similar to C. Taubes' work TT relating Gromov invariants to the SeibergWitten invariants of 4-manifolds, and our approach has been fundamentally influenced by Taubes. It is also similar to the 4-dimensional situation described by Lee and Parker in [LP1] and [LP2]. In both cases, the set of $J$-holomorphic embeddings in each homology class is discrete and compact for generic $J$ - a simplifying circumstance that does not appear to be true in the context of formula (0.1). Rather, for generic $J$ and with a fixed bound $E$ on area and genus, the moduli space $\mathcal{M}_{\text {emb }}(X)$ of embeddings is a countable set, possibly with accumulation points. With this picture in mind, our proof is based on three main ideas.

The first is the observation that, again for fixed $J$ and $E$, the full moduli space $\overline{\mathcal{M}}(X)$ can be decomposed (in many ways) into finitely many "clusters" $\mathcal{O}_{j}$. Each cluster consists of all of the $J$-holomorphic maps, including multiple covers, whose image lies in the $\varepsilon$-tubular neighborhood of some smooth, embedded $J$-holomorphic "core curve" $C \subset X$. A cluster is an open and closed subset of the moduli space; it may have complicated internal structure, but there is a well-defined total

The research of EI was supported in part by NSF grant DMS-0905738 and that of TP by NSF grant DMS-1011793. 
contribution $G W(\mathcal{O})$ of all the maps in the cluster to the series 0.1 . These contributions $G W(\mathcal{O})$ are local, depending only on $\varepsilon$ and $J$ in the neighborhood, and it suffices to show that the GV conjecture holds for the contribution of each cluster.

The second observation is that there exist certain standard "elementary clusters" whose local invariants are explicitly computable. Results of Junho Lee [L] show that, for each embedded genus $g$ curve $C$, there exists an almost complex structure $J$ in an $\varepsilon$ neighborhood $U$ of $C$ in $X$ that makes $C$ "super-rigid", meaning that all $J$-holomorphic maps into $U$ are in fact maps into $C$. For $g=0$, one can take $J$ to be the standard structure of the bundle $\mathcal{O}(-1) \oplus \mathcal{O}(-1)$, but for higher genus $J$ is a non-integrable almost complex structure. In Section 3 we compute the GW series $G W(\mathcal{O})$ of elementary clusters based on a calculation of Bryan and Pandharipande BP2. The resulting formula shows that the local version of the GV conjecture holds for elementary clusters.

The proof is completed by an isotopy argument in the spirit of Taubes' work, and extending arguments in IP1. For a fixed cluster, we deform $J$ in a neighborhood of the core curve to make it the $J$ of an elementary cluster. During the isotopy, the cluster series $G W\left(\mathcal{O}_{t}\right)$ can change according to several types of wall-crossing formulas. For a generic isotopy, the core curve could disappear in a "creation-annihilation" singularity. To avoid this, we use a generic isotopy in which the restriction of $J$ to the core curve is fixed; singularities then occur only when two core curves pass through one another momentarily. In Sections 6 and 7, we use Kuranishi models to show that the cluster series is invariant modulo contributions of finitely many clusters whose core curves have higher degree or genus. The Gopakumar-Vafa conjecture follows by induction.

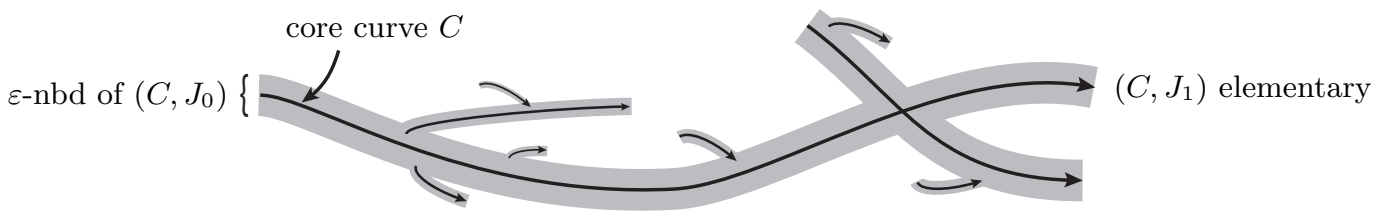

Figure 1. As $J_{t}$ changes, embedded curves of higher genus or degree can emerge from, or sink into, an $\varepsilon$-tubular neighborhood of $C$, and the core curve $C$ can pass through another embedded curve with the same genus and degree.

Our main results, Theorems 8.1 and 8.4 , can be stated as a structure theorem.

Structure Theorem. For any closed symplectic Calabi-Yau 6-manifold $X$, there exist unique integer invariants $e_{A, g}(X)$, indexed by non-zero classes $A \in H_{2}(X, \mathbb{Z})$ and genera $g \geq 0$, such that the series (0.1) has the form

$$
G W(X)=\sum_{A \neq 0} \sum_{g \geq 0} e_{A, g}(X) \cdot G W_{g}^{\text {elem }}\left(q^{A}, t\right),
$$

where $G W_{g}^{\text {elem }}(q, t)$ is the universal power series (3.4), which depends on $g$, but not on $X$. Furthermore, all coefficients $n_{A, g}$ in 0.1 are integers.

There is an extensive literature revolving around this GV conjecture. J. Bryan and R. Pandharipande have a series of papers about it, including two (BP1] and BP2]) relevant to our approach. For algebraic 3-folds, several BPS-type integer invariants have been defined using holomorphic bundles, including the Pandharipande-Thomas [PT] and Donaldson-Thomas invariants, with conjectural GV-type correspondences GW/DT/PT between them. For toric 3-folds, Maulik, Oblomkov, Okounkov and Pandharipande proved the GW/DT correspondence by calculating both sides explicitly in a computational tour de force $\mathrm{MOOP}$. Pandharipande and Pixton $\mathrm{PP}$ established the GW/PT correspondence for $\mathrm{CY}$ complete intersections in products of projective spaces. Other instances have been observed when a change of variables in the GW series produces integer invariants, including a formula for Fano classes $\left(A \in H_{2}(X)\right.$ with $\left.c_{1}(X) A>0\right)$ in symplectic 6-manifolds proved by A. Zinger [Z, and the computation of Klemm and Pandharipande for Calabi-Yau 4-folds [KP]. In 
Section 9 we combine our result on the Calabi-Yau classes first with Zinger's to obtain a GV-type formula for all symplectic 6-manifolds, and then with Klemm and Pandharipande's to obtain a GV-type formula for genus zero invariants of semipositive symplectic manifolds.

We thank the referees for their meticulous reviews and numerous insightful suggestions.

\section{Curves in symplectic Calabi-Yau 6-Manifolds}

The Gromov-Witten invariants of a closed symplectic manifold $(X, \omega)$ are constructed in two steps. One first forms the universal moduli space and its stabilization-evaluation map se

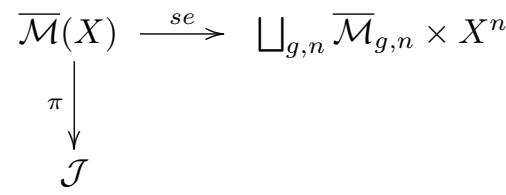

over a space $\mathcal{J}$ of $\omega$-tame almost complex structures on $X$. This moduli space consists of equivalence classes (up to reparametrizations of the domain) of pairs $(f, J)$, where $f: C \rightarrow(X, J)$ is a stable pseudo-holomorphic map whose domain $C$ is a nodal marked Riemann surface; it has components $\overline{\mathcal{M}}_{A, g, n}(X)$ labelled by the genus $g$ of $C$, the number $n$ of marked points, and the homology class $A=f_{*}[C] \in H_{2}(X ; \mathbb{Z})$ with $\omega(A) \geq 0$.

As is standard in the subject, we take $\mathcal{J}$ to be a space $\mathcal{J}^{l}$ of $C^{l}$ almost complex structures with $l$ large (and sometimes $\mathcal{J}=\mathcal{J}^{\infty}$ ), take $f$ in a corresponding space of maps (described in Section 4), and give $\overline{\mathcal{M}}(X)$ the Gromov topology. The results in Sections 1-3 depend on the specifics of these spaces only through Lemma 1.2 , whose rather technical proof is deferred until Section 5 and Appendix A.

It is frequently convenient to define the energy of a triple $(A, g, n)$ by

$$
E(A, g, n)=\max \{\omega(A), g, n\} \geq 0
$$

and to restrict attention to the subset $\overline{\mathcal{M}}^{E}(X)$ "below energy $E$ ", meaning the union of all components with $E(A, g, n) \leq E$, and the corresponding fibers $\overline{\mathcal{M}}^{J, E}(X)$ of 1.1 . The restriction of $\pi$ to each $\overline{\mathcal{M}}_{A, g, n}(X)$ is proper, and the fibers carry a $d$-dimensional virtual fundamental class

$$
\left[\overline{\mathcal{M}}_{A, g, n}^{J}(X)\right]^{\text {vir }} \in \check{\mathrm{H}}^{d}\left(\overline{\mathcal{M}}_{A, g, n}^{J}(X) ; \mathbb{Q}\right)^{\vee},
$$

where

$$
d=2 c_{1}(X) A+(\operatorname{dim} X-6)(1-g)+2 n,
$$

and $\check{H}^{*}(\cdot)^{\vee}$ denotes the dual of Čech cohomology with rational coefficients (cf. [Pd, Definition 9.3.1]). Moreover, 1.2 is deformation invariant in the sense that for every path $\gamma$ in $\mathcal{J}$ from $J_{0}$ to $J_{1}$,

$$
\left[\overline{\mathcal{M}}_{A, g, n}^{J_{0}}(X)\right]^{\text {vir }}=\left[\overline{\mathcal{M}}_{A, g, n}^{J_{1}}(X)\right]^{\text {vir }} \quad \text { in } \quad \check{\mathrm{H}}^{d}\left(\overline{\mathcal{M}}_{A, g, n}^{\gamma}(X)\right)^{\vee}
$$

(cf. the proof $\left[\mathrm{Pd}\right.$, Lemma 9.3.2]). Here $\overline{\mathcal{M}}^{\gamma}$ denotes the parameterized moduli space

$$
\overline{\mathcal{M}}^{\gamma}=\{(t,([f], J)) \in[0,1] \times \overline{\mathcal{M}}(X) \mid \gamma(t)=J\}
$$

obtained by pulling back $\overline{\mathcal{M}}$ along $\gamma$.

Gromov-Witten invariants are especially simple if $c_{1}(X)=0$ and $\operatorname{dim} X=6$; such spaces are often called symplectic Calabi-Yau 6-manifolds. In this case all terms in (1.3) vanish when $n=0$; the virtual fundamental class then has dimension $d=0$ for all $g$ and $A$, and we drop $n$ from the notation. In this case, the Gromov-Witten invariants

$$
G W_{A, g}(X)=\left\langle\left[\overline{\mathcal{M}}_{A, g}^{J}(X)\right]^{\text {vir }}, 1\right\rangle \in \mathbb{Q}
$$


are obtained by pairing the virtual fundamental class with 1 in $\check{\mathrm{H}}^{0}(\overline{\mathcal{M}})$. These are assembled in a formal power series

$$
G W(X)=\sum_{\omega(A)>0} \sum_{g=0}^{\infty} G W_{A, g}(X) t^{2 g-2} q^{A}
$$

in the "rational Novikov ring" $\Lambda$ generated by $t$ and $\left\{q^{A}\right\}$ with $t q^{A}=q^{A} t$ and $q^{A+B}=q^{A} q^{B}$, and whose elements have finitely many non-zero terms below each energy level. Note that the first sum in (1.6), over all positive $A \in H_{2}(X ; \mathbb{Z})$, omits any contributions from the class $A=0$. For consistency, the term " $J$-holomorphic map" will always mean a non-trivial map (i.e. $A \neq 0$ ), and a " $J$-holomorphic curve" in $X$ is the image of such a map. When $f$ is an embedding, we identify $C$ with its image $f(C)$ in $X$.

Remark 1.1. The use of virtual fundamental cycles here and in Section 2 makes the presentation clear and succinct, but is not essential for understanding the arguments in this paper. In fact, all of the symplectic manifolds $X$ that we consider are semipositive, and one might alternatively regard the GW invariants, and the local contributions to the GW invariants introduced in Section 2, as counts of perturbed $J$-holomorphic maps.

Using the terminology of [MS, $\S 2.5]$, a point $x \in C$ is called an injective point for a map $f: C \rightarrow X$ if $d f(x) \neq 0$ and $f^{-1}(f(x))=\{x\}$ when $C$ is smooth; if $C$ is nodal we also require that $x$ is not a node. A pseudo-holomorphic map $f: C \rightarrow X$ from a nodal (not necessarily connected) curve is simple if it has an injective point on each irreducible component. The open subset of simple maps in a moduli space will be denoted by the subscript simple; for example

$$
\overline{\mathcal{M}}^{J, E}(X)_{\text {simple }}
$$

denotes the set of simple maps in $\overline{\mathcal{M}}^{J, E}(X)$, while $\mathcal{M}(X)_{\text {simple }}$ denotes the open subset of $\overline{\mathcal{M}}(X)$ consisting of simple maps with smooth domain. The Micallef-White Theorem, [MW] or [MS, Prop. 2.5.1], implies that the set of injective points of a simple $J$-holomorphic map is open and dense in $C$, and hence the image under $f$ of this set is a submanifold of $X$.

A pair $p=(f, J)$ representing a point in $\overline{\mathcal{M}}^{J, E}(X)$ with smooth domain is regular if the linearization $D_{p}$, given by (4.13) and completed in Sobolev norms as in (4.26), is onto. It is a regular embedding if it is both regular and $f$ is an embedding. Since the index of $D_{p}$ is 0 by (1.3), each regular pair has a well-defined $\operatorname{sign}(f, J)= \pm 1$, given by the mod 2 spectral flow from $D_{p}$ to any invertible complex operator. Finally, by a Baire subset of the parameter space we mean a countable intersection of open and dense sets.

Lemma 1.2. Let $(X, \omega)$ be a symplectic Calabi-Yau 6-manifold. Then for each $E>0$ there is a Baire subset $\mathcal{J}_{E}^{*}$ of $\mathcal{J}$ such that for each $J \in \mathcal{J}_{E}^{*}$

(a) All simple $J$-holomorphic maps below energy $E$ are regular embeddings with disjoint images.

(b) The projection $\pi$ in (1.1) is a local diffeomorphism around each regular embedding.

Part (a) of Lemma 1.2 is proved as Corollary A.5 and part (b) is proved as Proposition 5.3a; these proofs use standard techniques. For our purposes, it is best to enlarge $\mathcal{J}_{E}^{*}$ to a set $\mathcal{J}_{\text {isol }}^{E}$ that emphasizes slightly weaker properties.

Definition 1.3. Denote by $\mathcal{J}_{\text {isol }}^{E}$ the set of all $J \in \mathcal{J}$ such that the moduli space (1.7), with the Gromov topology, consists of isolated points that are embeddings (not necessarily regular) with disjoint images.

Corollary 1.4. $\mathcal{J}_{\text {isol }}^{E}$ is dense in $\mathcal{J}$. 
Proof. For each $J \in \mathcal{J}_{E}^{*}$, each simple $J$-holomorphic map $f$ is an embedding, and is regular by part (a) of Lemma 1.2 By part (b), each such point $(f, J)$ is an isolated point of the fiber $\mathcal{M}^{J, E}(X)_{\text {simple }}$ of $\pi$.

Unfortunately, the images in $X$ of the pseudo-holomorphic maps that appear in Lemma 1.2 may accumulate. To focus on the images, consider the space $\operatorname{Subsets}(X)$ of all non-empty compact subsets of $X$. Fix a background Riemannian metric on $X$ with distance function $d$. Then $\operatorname{Subsets}(X)$ is a metric space with the Hausdorff distance, defined by

$$
d_{H}(A, B)=\sup _{a \in A} \inf _{b \in B} d(a, b)+\sup _{b \in B} \inf _{a \in A} d(a, b)
$$

for $A, B \subseteq X$. Let $c$ be the "underlying curve" map

$$
c: \overline{\mathcal{M}}(X) \longrightarrow \operatorname{Subsets}(X) \times \mathcal{J}
$$

that associates to each $(f, J)$ the pair $(f(C), J)$ with $f(C)$ regarded as subset of $X$. This map $c$ is continuous, and Gromov compactness implies that its restriction to $\overline{\mathcal{M}}^{E}(X)$ is proper. Let $\mathcal{C}(X)$, $\mathcal{C}^{E}$ and $\mathcal{C}^{J, E}$ denote, respectively, the images of $\overline{\mathcal{M}}(X), \overline{\mathcal{M}}^{E}(X)$ and $\overline{\mathcal{M}}^{J, E}(X)$ under $c$. With this notation, there is a commutative diagram

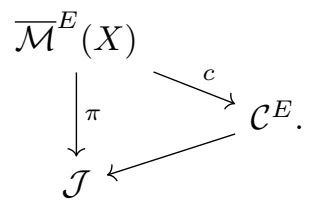

Viewed differently, convergence in $\mathcal{C}^{E}$ defines a topology on $\overline{\mathcal{M}}^{E}(X)$ that we will call the "rough topology".

In general, non-trivial $J$-holomorphic maps $f: C \rightarrow X$ from nodal curves can be multiple covers, and simple maps can converge to multiply covered maps. Definition 1.3 constrains how limits are multiply covered, as the following lemma shows.

Lemma 1.5. For $J \in \mathcal{J}_{\text {isol }}^{E}$,

(a) Every J-holomorphic map $f: C \rightarrow X$ below energy $E$ is a composition $\varphi \circ \rho$ of a holomorphic map $\rho: C \rightarrow C_{\text {red }}$ of complex curves and a J-holomorphic embedding $\varphi: C_{\text {red }} \rightarrow X$. This decomposition is unique up to reparametrizations of $C_{r e d}$. When $f$ is simple, $\varphi=f$.

(b) If $f: C \rightarrow X$ is a limit in the rough topology of a sequence $\left\{f_{n}\right\}$ in $\overline{\mathcal{M}}^{J, E}(X)$ with $d_{H}\left(f_{n}, f\right) \neq 0$ for all $n$, then the factorization $f=\varphi \circ \rho$ has either $\operatorname{deg} \rho>1$ or $\operatorname{genus}(C)>\operatorname{genus}\left(C_{\text {red }}\right)$.

Proof. The disjoint union of the irreducible components of $C$ is a smooth closed curve $\widetilde{C}$ called the normalization of $C$. Let $\widetilde{f}: \widetilde{C} \rightarrow X$ be the composition of the canonical map $\widetilde{C} \rightarrow C$ with $f$. The arguments of [MS, §2.5] show that $\widetilde{f}$ factors as $\widetilde{f}=\varphi \circ \widetilde{\rho}$ where $\varphi: C_{\text {red }} \rightarrow X$ is a simple $J$ holomorphic map from a smooth, possibly disconnected domain $C_{r e d}$, and $\widetilde{\rho}: \widetilde{C} \rightarrow C_{\text {red }}$ is a map of complex curves; $\widetilde{\rho}$ may take some components of $\widetilde{C}$ to points. But the assumption $J \in \mathcal{J}_{\text {isol }}^{E}$ implies that $C_{r e d}$ is connected and $\varphi$ is an embedding; $\widetilde{\rho}$ then descends to a holomorphic map $\rho: C \rightarrow C_{\text {red }}$, unique up to reparametrization, with $f=\varphi \circ \rho$.

Part (b) immediately follows from Gromov compactness and the fact that simple maps are isolated for $J \in \mathcal{J}_{\text {isol }}^{E}$, and therefore the limit map $f=\varphi \circ \rho$ is not simple.

To each class $A \in H_{2}(X, \mathbb{Z})$ in the positive cone $\omega(A)>0$ we associate a positive integer

$$
d(A)=\ell c m\left\{k \in \mathbb{N}_{+} \mid A=k B \text { where } B \in H_{2}(X, \mathbb{Z})\right\}
$$

called the degree of $A$, where $\ell c m$ denotes the lowest common multiple. Let $\Omega(d)$ be the number of prime factors of $d$, counted with multiplicity. For any map $f$ from a genus $g$ curve representing a 
degree $d$ class, we define its level to be

$$
\ell(f)=\Omega(d)+g
$$

The components of the moduli space are filtered by the degree and genus, and therefore by their level; the level filtration will be used frequently in later sections. For each $E$, the sets

$$
\overline{\mathcal{M}}_{m}^{E}(X)=\left\{(f, J) \in \overline{\mathcal{M}}^{E}(X) \mid \ell(f) \leq m\right\}
$$

filter $\overline{\mathcal{M}}^{E}(X)$, and their images under 1.9

$$
\mathcal{C}_{m}^{E}=c\left(\overline{\mathcal{M}}_{m}^{E}(X)\right)
$$

filter the image $\mathcal{C}^{E}=c\left(\overline{\mathcal{M}}^{E}(X)\right)$.

For each $J \in \mathcal{J}_{\text {isol }}^{E}$, the map $c$, when applied to multiply-covered maps, decreases the level but respects the filtration; for such $J$ the fiber $\mathcal{C}_{m}^{J, E}$ of $\mathcal{C}_{m}^{E} \rightarrow \mathcal{J}$ is the collection of embedded $J$ holomorphic curves with level at most $m$. In this notation, $m=0$ corresponds to genus zero curves representing primitive classes, and $\mathcal{C}_{m}^{E}$ is the collection of embedded pseudo-holomorphic curves in $X$ with $g+\Omega(d) \leq m$ and energy at most $E$.

Lemma 1.6. For any fixed $J \in \mathcal{J}_{\text {isol }}^{E}$,

(a) $\mathcal{C}_{m}^{J, E} \subseteq \mathcal{C}_{m+1}^{J, E}$ is a filtration of $\mathcal{C}^{J, E}$ with $\mathcal{C}_{m}^{J, E}=\mathcal{C}^{J, E}$ for $m$ large.

(b) $\mathcal{C}_{m}^{J, E}$ and $\mathcal{C}^{J, E}=\bigcup \mathcal{C}_{m}^{J, E}$ are compact countable subsets of the metric space $\mathcal{C}(X)$.

(c) For any neighborhood $U$ of $\mathcal{C}_{m-1}^{J, E}$, the set $\mathcal{C}_{m}^{J, E} \backslash U$ is a finite collection of embedded J-holomorphic curves.

In particular, there are finitely many genus zero J-holomorphic curves with energy less than $E$ representing primitive classes.

Proof. The inclusion in (a) is true by definition, and the second part of (a) holds because, by Gromov compactness, only finitely many homology classes are represented by $J$-holomorphic maps below energy $E$.

Next, each set $\mathcal{C}_{m}^{J, E}$ is compact because it is the image of a compact set, namely the fiber $\overline{\mathcal{M}}_{m}^{J, E}(X)$ of 1.11), under the continuous map (1.9). Then $\mathcal{C}_{m}^{J, E} \backslash U$ is a closed subset of the compact metric space $\mathcal{C}_{m}^{J, E}$, so any infinite sequence $\left\{C_{i}\right\}$ has an accumulation point $C_{0}$. Because only finitely many homology classes are represented by $J$-holomorphic maps below energy $E$ we may assume, after passing to a subsequence, that they all have the same genus and same homology class $\left[C_{i}\right]=k \beta$ for the same primitive class $\beta$ and the same $k$ with $\Omega(k)+g \leq m$. By Lemma 1.5 the limit is a multiple cover of a curve of a strictly lower level, but that's impossible because $U$ is open. Thus $\mathcal{C}_{m}^{J, E} \backslash U$ is finite. Finally, taking $U_{k}$ to be the $1 / k$ tubular neighborhood of $\mathcal{C}_{m-1}^{J, E}$, we conclude that $\mathcal{C}_{m}^{J, E} \backslash \mathcal{C}_{m-1}^{J, E}=\bigcup_{k}\left(\mathcal{C}_{m}^{J, E} \backslash U_{k}\right)$ is countable, and hence $\mathcal{C}_{m}^{J, E}$ and $\mathcal{C}^{J, E}$ are countable.

\section{Clusters in Symplectic Manifolds}

Now suppose that $X$ is a symplectic Calabi-Yau 6-manifold (we retain this assumption until Section 4). For each subset $S \subset \mathcal{J}$, we can consider the moduli space $\overline{\mathcal{M}}^{S}(X)=\pi^{-1}(S)$ over $S$ or, with an energy bound,

$$
\overline{\mathcal{M}}^{S, E}(X)=\left\{([f], J) \mid[f] \in \overline{\mathcal{M}}^{J, E}(X), J \in S\right\} .
$$

A decomposition of the moduli space $\overline{\mathcal{M}}^{S, E}(X)$ is a way of writing it as a finite disjoint union $\bigsqcup_{i} \mathcal{O}_{i}$ of subsets $\mathcal{O}_{i}$ that are both open and closed in the Gromov topology. Given such a decomposition 
and a compact subset $V$ of $S$, the sets $\left\{\mathcal{O}_{i} \cap \pi^{-1}(V)\right\}$ are a decomposition of $\overline{\mathcal{M}}^{V, E}(X)$, giving a natural isomorphism

$$
\check{\mathrm{H}}^{*}\left(\overline{\mathcal{M}}^{V, E}(X)\right)^{\vee} \cong \bigoplus_{i} \check{\mathrm{H}}^{*}\left(\mathcal{O}_{i} \cap \pi^{-1}(V)\right)^{\vee}
$$

Note that for every $J \in S$ and every $A, g$ with energy at most $E$, the inclusion $\overline{\mathcal{M}}_{A, g}^{J}(X) \hookrightarrow \overline{\mathcal{M}}^{J, E}(X)$ induces a map

$$
\check{\mathrm{H}}^{*}\left(\overline{\mathcal{M}}_{A, g}^{J}(X)\right)^{\vee} \rightarrow \check{\mathrm{H}}^{*}\left(\overline{\mathcal{M}}^{J, E}(X)\right)^{\vee} .
$$

whose image is the lefthand side of (2.1) with $V=\{J\}$. The image of virtual fundamental class $\left[\overline{\mathcal{M}}_{A, g}^{J}(X)\right]^{v i r}$ then decomposes under 2.1 into a sum of components

$$
\operatorname{pr}_{i}\left[\overline{\mathcal{M}}_{A, g}^{J}(X)\right]^{v i r} \in \check{\mathrm{H}}^{*}\left(\mathcal{O}_{i} \cap \pi^{-1}(J)\right)^{\vee} .
$$

As in 1.5$)$, we set

$$
G W_{A, g}\left(\mathcal{O}_{i} \cap \pi^{-1}(J)\right)=\left\langle p r_{i}\left[\overline{\mathcal{M}}_{A, g}^{J}(X)\right]^{v i r}, 1\right\rangle \in \mathbb{Q}
$$

and define the contribution of $\mathcal{O}_{i} \cap \pi^{-1}(J)$ to the GW series to be the sum of the form (1.6) whose coefficients are given by 2.2 for all $A, g$ with energy at most $E$, and are 0 otherwise. Then

$$
G W^{E}(X)=\sum_{i} G W^{E}\left(\mathcal{O}_{i} \cap \pi^{-1}(J)\right)
$$

where, on both sides, $G W^{E}$ denotes the $G W$ series truncated at energy $E$.

Equation (1.4) implies that the coefficients 2.2 are deformation invariant, as follows. For any path $\gamma$ in $S$ from $J_{0}$ to $J_{1}$, and any $A, g$ with energy at most $E$, we can take $V$ to be the image of $\gamma$, and consider the map

$$
\overline{\mathcal{M}}_{A, g}^{\gamma}(X) \rightarrow \overline{\mathcal{M}}^{V, E}(X)
$$

induced by $(t,[f], J) \mapsto([f], J)$. The equality (1.4) pushes forward by this map to give an equality in the group on the lefthand side of 2.1). Applying the isomorphism 2.1) and projecting onto the $i$ 'th component then shows that the coefficients $(2.2)$ for $J=J_{0}$ and $J=J_{1}$ are equal.

Lemma 2.1. Each open subset $U$ of $\mathcal{C}(X)$ with $\partial U \cap \mathcal{C}^{J, E}=\emptyset$ has a well-defined contribution $G W^{E}(U, J)$ to $G W^{E}(X)$. The collection of $J$ for which $\partial U \cap \mathcal{C}^{J, E}=\emptyset$ is open in $\mathcal{J}$, and the contribution $G W^{E}(U, J)$ is locally constant as a function of $J$.

Proof. The assumption implies that the intersections of both $U$ and its complement $U^{c}$ with $\mathcal{C}^{J, E}$ are open subsets of the image $\mathcal{C}^{J, E}$ of $c$. Since $c$ is continuous, $\mathcal{O}=c^{-1}\left(U \cap \mathcal{C}^{J, E}\right)$ and $\mathcal{O}^{c}=$ $c^{-1}\left(U^{c} \cap \mathcal{C}^{J, E}\right)$ are open and closed subsets of $\overline{\mathcal{M}}^{J, E}(X)$. Define $G W^{E}(U, J)$ to be the contribution $G W^{E}\left(\mathcal{O} \cap \pi^{-1}(J)\right)$ of $\mathcal{O}$ to the sum 2.3 associated to the decomposition $\mathcal{O} \sqcup \mathcal{O}^{c}$.

Next note that the condition $\partial U \cap \mathcal{C}^{\mathcal{J}} E=\emptyset$ is an open condition on $J$. To see why, fix $U$ and $E$. If this condition held for some $J$, but failed to hold for a sequence $J_{k} \rightarrow J$ in $\mathcal{J}$, there would be a sequence of $J_{k}$-holomorphic curves $C_{k}$ in $\partial U$ with energy $E(A, g, n)$ bounded by $E$. Applying Gromov compactness, one could find a subsequence converging to a $J$-holomorphic curve $C$. But $\partial U$ and $\mathcal{C}^{J, E}$ are both closed in the Gromov topology, so the limit curve $C$ would lie in $\partial U \cap \mathcal{C}^{J, E}$, contradicting the hypothesis.

Therefore there exists a ball $V$ around $J$ in $\mathcal{J}$ such that $\partial U \cap \mathcal{C}^{E, V}=\emptyset$. This gives rise to the same decomposition $\mathcal{O} \sqcup \mathcal{O}^{c}$ but now of the moduli space $\overline{\mathcal{M}}^{V, E}(X)$ over the entire ball $V$, therefore the contribution of $U$ is defined for each $J \in V$ and it is constant on $V$. 
The geometric content of the contribution $G W^{E}(U, J)$ is most clearly seen by choosing $U$ of the form $B(C, \varepsilon) \times \mathcal{J}$ for a ball $B(C, \varepsilon)$ of small radius $\varepsilon$ in the Hausdorff distance centered at a $J$ holomorphic curve $C$. The subset of $\overline{\mathcal{M}}^{J}(X)$ that lies in $c^{-1}(U)$ is then a collection of $J$-holomorphic maps whose images are uniformly $\varepsilon$-close to $C$ in $X$. We will call such a collection a cluster if the following properties hold.

Definition 2.2. A cluster $\mathcal{O}=(C, \varepsilon, J)$ in $X$ below energy $E$ (an "E-cluster") consists of an almost complex structure $J \in \mathcal{J}$, an embedded $J$-holomorphic curve $C$ and a radius $\varepsilon>0$ with the following properties:

(a) All non-constant $J$-holomorphic maps in the ball $B(C, \varepsilon)$ with energy $\leq E$ represent $k[C]$ for some $k \geq 1$, and have genus $g \geq g(C)$;

(b) $C$ is the only $J$-holomorphic map in its degree and genus in the ball $B(C, \varepsilon)$.

(c) There are no J-holomorphic curves with energy $\leq E$ at precisely $\varepsilon$ Hausdorff distance from $C$.

The curve $C$ is called the core of the cluster. Note that, by the definition of Hausdorff distance, curves in $B(C, \varepsilon)$ lie in the $\varepsilon$-tubular neighborhood of $C$ in $X$.

The next lemma shows that small balls in the Hausdorff metric are often clusters. In fact, conditions (a) and (b) of Definition 2.2 are automatic for small $\varepsilon$ when $C$ is regular. Condition (c) is the important one: it implies that $\mathcal{O}=(C, \varepsilon, J)$ has a well-defined contribution

$$
G W^{E}(\mathcal{O}) \in \Lambda \text {. }
$$

Lemma 2.3 (Cluster existence). For each $J \in \mathcal{J}_{\text {isol }}^{E}(X)$ and each simple J-holomorphic curve $C$, the set $S$ of $\varepsilon>0$ for which the ball $B(C, \varepsilon)$ is an E-cluster is open and dense in a non-empty interval $\left[0, \varepsilon_{C}\right]$, and the contribution (2.4) is locally constant on $S$.

Proof. By definition, for any $J \in \mathcal{J}_{\text {isol }}^{E}(X)$, all simple $J$-holomorphic curves are embedded and isolated in their degree and genus. Since $\mathcal{C}^{J, E}$ is compact, and an embedded curve $C$ can appear as an accumulation point only of curves representing $k[C]$ and having genus at least that of $C$, Lemma 1.5 implies that there is an $\varepsilon_{C}>0$ such that conditions (a) and (b) of Definition 2.2 hold for all $\varepsilon \leq \varepsilon_{C}$.

Finally, by Lemma 1.6 , the image of $\mathcal{C}^{J, E}$ under the distance function $B\left(C, \varepsilon_{C}\right) \rightarrow\left[0, \varepsilon_{C}\right]$ is countable and compact, so its complement - which is the set of $\varepsilon$ that satisfy condition (c) of Definition 2.2 - is open and dense.

Proposition 2.4 (Cluster decompositions). Given $E$ and $J \in \mathcal{J}_{\text {isol }}^{E}$, each open subset $U$ of $\mathcal{C}(X)$ such that $\partial U \cap \mathcal{C}^{J, E}=\emptyset$ has a finite E-cluster decomposition $\left\{\mathcal{O}_{i}=\left(C_{i}, J, \varepsilon_{i}\right)\right\}$, and hence

$$
G W^{E}(U)=\sum_{i} G W^{E}\left(\mathcal{O}_{i}\right) .
$$

Proof. We will inductively construct cluster decompositions of the sets $U_{m}=U \cap \mathcal{C}_{m}^{J, E}$, beginning with the trivial case $U_{-1}=\emptyset$. This induction is finite because $\mathcal{C}_{m}^{J, E}=\mathcal{C}^{J, E}$ for $m$ sufficiently large by Lemma 1.6 a.

Suppose that $\left\{B_{i}=B\left(C_{i}, \varepsilon_{i}\right)\right\}$ is a cluster decomposition of $U_{m}$. This means that the balls $B_{i}$ are disjoint, that the compact set $U_{m}$ lies in $V=\bigsqcup B_{i}$, and there are no $J$-holomorphic curves on $\partial V=\bigsqcup \partial B_{i}$. Lemma 1.6 shows that $U_{m+1} \backslash V$ is a finite collection of curves $\left\{C_{j}\right\}$. None of these $C_{j}$ lie on $\partial V$, so we can choose radii $\varepsilon_{j}>0$ such that the balls $B_{j}^{\prime}=B\left(C_{j}, \varepsilon_{j}\right)$ are clusters (by Lemma 2.3 and are disjoint from each other and from the balls $B_{i}$. These clusters $B_{j}^{\prime}$, together with the original $B_{i}$ are a cluster decomposition of $U_{m+1}$, completing the induction step.

Corollary 2.5 (Cluster refinement). Fix any cluster $\mathcal{O}=(C, J, \varepsilon)$ with $J \in \mathcal{J}_{\text {isol }}^{E}$. For any $\varepsilon^{\prime} \in(0, \varepsilon)$ for which $\mathcal{O}^{\prime}=\left(C, J, \varepsilon^{\prime}\right)$ is a cluster, there exists finitely many higher level clusters $\left\{\mathcal{O}_{i}=\left(C_{i}, J, \varepsilon_{i}\right)\right\}$ 
such that

$$
G W^{E}(\mathcal{O})=G W^{E}\left(\mathcal{O}^{\prime}\right)+\sum_{i} G W^{E}\left(\mathcal{O}_{i}\right)
$$

Proof. Consider $U=B(C, \varepsilon) \backslash B\left(C, \varepsilon^{\prime}\right)$. Because both $\mathcal{O}, \mathcal{O}^{\prime}$ are clusters, (a) there are no curves in $\mathcal{C}^{J, E}$ on $\partial U$ and (b) all the curves in $\mathcal{C}^{J, E} \cap U$ have strictly higher level compared to that of $C$ (as $C$ is the only bottom level curve in both clusters). By Proposition 2.4. there exists a cluster decomposition 2.5) of $U$, where the $\mathcal{O}_{i}$ are strictly higher level. But condition (a) also implies that $U$ and $\mathcal{O}^{\prime}$ give a decomposition of $\mathcal{O}$ so $G W(\mathcal{O})=G W\left(\mathcal{O}^{\prime}\right)+G W(U)$, which implies 2.6).

\section{Elementary Clusters AND their CONTRIBUtions}

The GW series can be explicitly calculated for one very special type of embedded curve. In this section we describe how this can be done by combining ideas already in the literature. We first construct such "elementary curves" using a remarkable non-integrable almost complex structure discovered by Junho Lee $[\mathrm{L}$, and then point out that the GW series for these curves has been calculated by J. Bryan and R. Pandharipande [BP2].

Definition 3.1. In a symplectic Calabi-Yau 6-manifold $X$, a cluster $\mathcal{O}=(C, \varepsilon, J)$ is called elementary if

(a) The core curve $C$ is balanced, meaning that its normal bundle splits as $N_{C}=L \oplus L$ in such a way that the normal operator $D^{N}$, given by (4.15) below, splits as $D^{\prime} \oplus D^{\prime}$.

(b) The only non-trivial J-holomorphic maps into $B(C, \varepsilon)$ are multiple covers of the embedding $C \hookrightarrow X$.

(c) For each such cover $\rho$, the pullback operator $\rho^{*} D^{\prime}$ is injective ( $C$ is super-rigid).

Property (c) implies that the core $C$ of an elementary cluster is a regular $J$-holomorphic map, and one can show it also implies (b) for sufficiently small $\varepsilon>0$ by the rescaling argument of [IP2]. Property (a) allows us to actually calculate the GW contribution.

When $C$ is a rational curve, the unit disk bundle in $\mathcal{O}(-1) \oplus \mathcal{O}(-1)$ is an elementary cluster. The following proposition uses non-integrable almost complex structures to construct similar examples for any curve. The proof begins with the choice of a spin structure on $C$, i.e. a holomorphic line bundle $L \rightarrow C$ together with a (holomorphic) identification of $L^{2}$ with the canonical bundle $K_{C}$ of C.

Proposition 3.2. For every smooth complex curve $C$, there exists an elementary cluster whose core is $C$.

Proof. Fix a curve $C$ of genus $g$, a spin structure $L$, and a Kähler structure $(J, g, \omega)$ on the total space $Y$ of $L \oplus L \rightarrow C$ compatible with its holomorphic structure. The canonical bundle $K_{Y}=$ $\pi^{*}\left(L^{-2} \otimes K_{C}\right)$ of $Y$ is then trivial, so $Y$ is a Calabi-Yau 6-manifold. We will perturb $J$ to obtain an almost complex structure on the unit disk bundle $U \subset Y$ that makes $U$ an elementary cluster.

First consider the total space $Z$ of $p: L \rightarrow C$. Its canonical bundle $K_{Z}=T^{2,0} Z$ is the pullback $p^{*}\left(L^{-1} \otimes K_{C}\right)=p^{*}(L)$, which has a canonical section $\beta$ that vanishes transversally along the zero section. Pullback $\beta$, regarded as a 2 -form, by the bundle projections $p_{1}, p_{2}: Y \rightarrow Z$ onto the first and second copy of $L$ and set $\alpha=p_{1}^{*} \beta+p_{2}^{*} \beta$. Then $\alpha$ is a closed $(2,0)$-form on $Y$ that vanishes to first order along the zero section of $L \oplus L$, which is the core curve $C$ of the disk bundle $U \subset Y$. Following Junho Lee, define a bundle map $K_{\alpha}: T Y \rightarrow T Y$ by $g\left(u, K_{\alpha} v\right)=(\alpha+\bar{\alpha})(u, v)$ for all $u, v \in T Y$ and set

$$
J_{\alpha}=\left(I d+J K_{\alpha}\right)^{-1} J\left(I d+J K_{\alpha}\right) .
$$


Then $J_{\alpha}$ is an $\omega$-tame almost complex structure on $U$ after replacing $\alpha$ by $t \alpha$ for small $t>0$ (cf. Section 2 of $(\mathrm{L})$. In this context, Lee proved that the image of every non-trivial $J_{\alpha}$-holomorphic map into $D$ is everywhere tangent to ker $K_{\alpha}$ (cf. [L, (2.4)]). It is straightforward to check that at each point $p \in Y$ not on the zero section, $\operatorname{ker} K_{\alpha}$ is vertical. Consequently, any map whose image is tangent to $\operatorname{ker} K_{\alpha}$ lies in a fiber of $Y \rightarrow C$ or in the zero section. We conclude that every $J_{\alpha^{-}}$ holomorphic map into $U$ that represents $k[C]$ for some $k \neq 0$ is a map into the core curve $C$ of the disk bundle $U$.

Along the zero section of $Y$ and for $v \in T C, \nabla_{v} K_{\alpha}$ decomposes under the splitting $T C \oplus L \oplus L$ as $0 \oplus \nabla_{v} K_{\beta} \oplus \nabla_{v} K_{\beta}$. Correspondingly, the normal operator $D^{N}$, given by 4.15), splits as $D_{\beta}^{N} \oplus D_{\beta}^{N}$ as can be seen from [LP1, (8.4)]. As in Section 4 of [LP1], the normal projection of $D_{\beta}$ is the sum $\bar{\partial}+R_{\beta}$ of the $\bar{\partial}$-operator on $L$ and a bundle map $R_{\beta}: L \rightarrow T^{0,1} C \otimes L$ that satisfies $J R_{\beta}=-R_{\beta} J$. The injectivity condition (c) of Definition 3.1 is then exactly the statement of Proposition 8.6 of LP1.

By Lemma 2.1 an elementary cluster has a well-defined $\Lambda$-valued series $G W(\mathcal{O})$, independent of $\varepsilon$. As usual, there is an associated disconnected invariant

$$
Z(\mathcal{O})=\exp (G W(\mathcal{O}))
$$

obtained by exponentiating in the Novikov ring. It turns out that $Z(\mathcal{O})$ is the more easily calculated.

Proposition 3.3. The disconnected $G W$ invariant of an elementary cluster $\mathcal{O}$ whose core $C$ has genus $g$ is given by

$$
Z(\mathcal{O})=1+\sum_{d \geq 1} \sum_{\mu \vdash d} \prod_{\square \in \mu}\left(2 \sin \frac{h(\square) t}{2}\right)^{2 g-2} q^{d C},
$$

where the second sum is over all partitions $\mu$ of $d$, the product is over the boxes in the Ferrers diagram of $\mu$, and $h(\square)$ is the hooklength of $\square \in \mu$.

Proof. Because the linearization on covers of the core curve $C$ is injective, the contribution to the GW invariant of its multiple covers can be calculated using the Euler class of Taubes obstruction bundle (this is the 6-dimensional version of the setup in [LP2], and a special case of Theorem 1.2 in [Z]).

Consider the moduli space $\overline{\mathcal{M}}_{d, \chi}^{\circ}(C)$ of degree $d$ holomorphic maps $\rho$ to $C$ whose domain is possibly disconnected and has Euler characteristic $\chi$, and where $\rho$ is non-trivial on each connected component. This carries a virtual fundamental cycle $\left[\overline{\mathcal{M}}_{d, \chi}^{\circ}(C)\right]^{\text {vir }}$ of even complex dimension $b=d(2-2 g)-\chi$. The operators $D^{N}$ and $D^{\prime}$ of Definition 3.1 induce families of real operators $\mathcal{D}^{N}$ and $\mathcal{D}^{\prime}$ over $\overline{\mathcal{M}}_{d, \chi}^{\circ}(C)$ whose fibers at $\rho$ are the pullback operators $\rho^{*} D^{N}$ and $\rho^{*} D^{\prime}$. By Definition 3.1 , the corresponding index bundles satisfy Ind $\mathcal{D}^{N}=$ Ind $\mathcal{D}^{\prime} \oplus$ Ind $\mathcal{D}^{\prime}$. A priori, these are real virtual bundles, but Definition 3.1k insures that the Taubes obstruction bundle $O b=-\operatorname{Ind}_{\mathbb{R}} \mathcal{D}^{N}$ is an actual vector bundle of rank $b$, equal to the direct sum of two copies of $O b^{\prime}=-\operatorname{Ind}_{\mathbb{R}} \mathcal{D}^{\prime}$. The bundles $O b$ and $O b^{\prime}$ each come with a canonical orientation determined, on each connected component of the space of covers, by the spectral flow to an injective complex operator over one fixed cover $\rho$. Computing this spectral flow along a path of operators that respect the direct sum decomposition, one sees that $O b=O b^{\prime} \oplus O b^{\prime}$ as oriented real bundles.

With this notation, the elementary contribution is equal to the integral of the Euler class

$$
Z_{d, \chi}(\mathcal{O})=\int_{\left[\overline{\mathcal{M}}_{d, \chi}^{\circ}(C)\right]^{v i r}} e(O b)
$$

where

$$
e(O b)=e\left(O b^{\prime} \oplus O b^{\prime}\right)=e\left(O b^{\prime}\right) \cup e\left(O b^{\prime}\right)=(-1)^{b / 2} c_{b}\left(O b^{\prime} \otimes_{\mathbb{R}} \mathbb{C}\right) .
$$


This Chern class factors through $K$-theory (in general Euler classes do not). Because $D^{\prime}=\bar{\partial}_{L}+R$ is a 0 'th order deformation of the complex operator $\bar{\partial}_{L}$, the complexification of the index bundles of $D^{\prime}$ and $\bar{\partial}_{L}$ are equal in $K$-theory, so

$$
c_{b}\left(O b^{\prime} \otimes_{\mathbb{R}} \mathbb{C}\right)=c_{b}\left(- \text { Ind } \bar{\partial}_{L} \otimes_{\mathbb{R}} \mathbb{C}\right)=c_{b}\left(- \text { Ind } \bar{\partial}_{L} \oplus\left(-\operatorname{Ind} \bar{\partial}_{L}\right)^{*}\right) .
$$

Combining the last three displayed equations gives

$$
Z_{d, \chi}(\mathcal{O})=(-1)^{b / 2} \int_{\left[\overline{\mathcal{M}}_{d, \chi}^{\circ}(C)\right]^{v i r}} c_{b}\left(- \text { Ind } \bar{\partial}_{L} \oplus\left(-\operatorname{Ind} \bar{\partial}_{L}\right)^{*}\right) .
$$

The right-hand side of 3.2 can be evaluated using equivariant techniques. The torus $T=\mathbb{C}^{*} \times \mathbb{C}^{*}$ acts on the total space $Y$ of the holomorphic bundle $N_{C}=L \oplus L$. With the antidiagonal $\mathbb{C}^{*}$-action, $Y$ is an equivariant local Calabi-Yau 3-fold. Bryan and Pandharipande defined a 'residue' generating function $Z^{T}(Y)$ whose coefficients

$$
Z_{d, \chi}^{T}(Y)=\int_{\left[\overline{\mathcal{M}}_{d, \chi}^{\circ}(C)\right]^{v i r}} c_{b}\left(-\operatorname{Ind} \bar{\partial}_{L \oplus L}\right)
$$

are equivariant integrals (defined by localization). They proceeded to express them in terms of ordinary integrals:

$$
Z_{d, \chi}^{T}(Y)=\sum_{b_{1}+b_{2}=b} \int_{\left[\overline{\mathcal{M}}_{d, \chi}^{\circ}(C)\right]^{v i r}}\left(t_{1} / t_{2}\right)^{\left(b_{2}-b_{1}\right) / 2} c_{b_{1}}\left(- \text { Ind } \bar{\partial}_{L}\right) c_{b_{2}}\left(- \text { Ind } \bar{\partial}_{L}\right)
$$

where $t_{1}, t_{2}$ are the weights of the action (cf. page 105 of BP2]). For the antidiagonal action $t_{1}=-t_{2}$, (3.3) reduces to (3.2) after noting that $c_{k}\left(E^{*}\right)=(-1)^{k} c_{k}(E)$ for $E=-$ Ind $\bar{\partial}_{L}$. On the other hand, for the antidiagonal action, Bryan and Pandharipande also explicitly calculated (3.3) to be the coefficient of the series appearing on the right hand side of (3.1) (Corollary 7.3 of [BP2]). This completes the proof.

The series (3.1) is a universal power series that depends only on the genus $g$ of $C$. Thus we set

$$
G W_{g}^{\text {elem }}(q, t)=\log Z_{g}^{\text {elem }}(q, t),
$$

where

$$
Z_{g}^{\text {elem }}(q, t)=1+\sum_{d \geq 1} \sum_{\mu \vdash d} \prod_{\square \in \mu}\left(2 \sin \frac{h(\square) t}{2}\right)^{2 g-2} q^{d} .
$$

In fact, taking $\log$ of 3.5 and separating the $d=1$ term of the series,

$$
G W_{g}^{\text {elem }}(q, t)=q\left(2 \sin \frac{t}{2}\right)^{2 g-2}+\sum_{d \geq 2} \sum_{h \geq g} G W_{d, h}(g) q^{d} t^{2 h-2},
$$

for some coefficients $G W_{d, h}(g) \in \mathbb{Q}$. Since the coefficient of the leading term $q t^{2 g-2}$ is +1 , the core curve $C$ of any elementary cluster has $\operatorname{sign} C>0$.

Now apply the "BPS transform", which takes an arbitrary element of the Novikov ring to another by

$$
\sum_{A, g} N_{A, g} t^{2 g-2} q^{A}=\sum_{A, g} n_{A, g} \sum_{k=1}^{\infty} \frac{1}{k}\left(2 \sin \frac{k t}{2}\right)^{2 g-2} q^{k A} .
$$

This transform is well-defined and invertible (Proposition 2.1 of [BP1]). Thus for an elementary cluster $\mathcal{O}$ whose core $C$ has genus $g$ we can write

$$
G W(\mathcal{O})=G W_{g}^{\text {elem }}\left(q^{C}, t\right)=\sum_{d \neq 0} \sum_{h} n_{d, h}(g) \sum_{k=1}^{\infty} \frac{1}{k}\left(2 \sin \frac{k t}{2}\right)^{2 h-2} q^{k d C}
$$


for uniquely determined coefficients $n_{d, h}(g)$ that are, a priori, rational numbers. These coefficients have been explicitly calculated for low degree $(d \leq 2)$ and for low genus $(g \leq 1)$. A combinatorics argument now handles the case $g \geq 2$, yielding a basic fact:

Proposition 3.4. The local Gopakumar-Vafa conjecture is true for elementary clusters. More specifically, the coefficients of the series (3.7) associated with a genus g elementary cluster $\mathcal{O}$ satisfy

(a) (Integrality) $n_{d, h}(g) \in \mathbb{Z}$.

(b) (Finiteness) for each $d$ fixed, $n_{d, h}(g)=0$ for $h<g$ or $h$ large.

(c) For $g=0$, all $n_{d, h}(g)$ vanish except $n_{1,0}(g)=1$.

(d) For $g=1$, all $n_{d, h}(g)$ vanish except $n_{d, 1}(g)=1$ for each $d \geq 1$.

Proof. When the core curve has genus zero and normal bundle $O(-1) \oplus O(-1), \mathcal{O}$ is an elementary cluster and its contribution to the $G W$ invariant was first calculated by Faber and Pandharipande. Specifically, letting $c(h, d)$ denote the coefficient of $q^{d} t^{2 h-2}$ in $(3.6)$ with $g=0$, equations (34), (35) and the middle displayed equation on page 192 of $[\mathrm{FP}$ imply the formulas

$$
c(h, d)=d^{2 h-3} c(h, 1) \quad \sum c(h, 1) t^{2 h-2}=\left(2 \sin \left(\frac{t}{2}\right)\right)^{-2}
$$

(these are equations (1) and (2) in $[\mathrm{P}]$ ). Consequently, the genus 0 elementary GW series is

$$
G W_{0}^{\text {elem }}(q, t)=\sum_{h, k} c(h, k) t^{2 h-2} q^{k}=\sum_{k} \frac{1}{k}\left(2 \sin \frac{k t}{2}\right)^{-2} q^{k} .
$$

Comparing with (3.7) gives (c).

For genus $g=1,3.5$ reduces to the generating function for the number $p(d)$ of unordered partitions of $d$ :

$$
Z_{1}^{\text {elem }}(q, t)=1+\sum_{d \geq 1} p(d) q^{d}=\prod_{d=1}^{\infty}\left(\frac{1}{1-q^{d}}\right) .
$$

Hence

$$
G W_{1}^{\text {elem }}(q, t)=\log Z_{1}^{\text {elem }}(q, t)=-\sum_{d=1}^{\infty} \log \left(1-q^{d}\right)=\sum_{d=1}^{\infty} \sum_{k=1}^{\infty} \frac{q^{k d}}{k} .
$$

Comparing with (3.7) gives (d).

In the higher genus case, both (a) and (b) are consequences of an algebraic fact about power series with integral coefficients that follows by combining several results in the paper [PT] of Pandharipande and Thomas. Making the change of variable $Q=e^{i t},(3.7)$ becomes

$$
\log Z_{g}^{\text {elem }}=\sum_{d \geq 1} \sum_{h} n_{d, h}(g) \sum_{k \geq 1} \frac{(-1)^{h-1}}{k}\left(Q^{k}+Q^{-k}-2\right)^{h-1} q^{k d} .
$$

On the other hand, for $g \geq 1$, 3.5 becomes

$$
Z_{g}^{\text {elem }}=1+\sum_{d \geq 1} \sum_{\mu \vdash d} \prod_{\square \in \mu}(-1)^{g-1}\left(Q^{h(\square)}+Q^{-h(\square)}-2\right)^{g-1} q^{d}=\sum_{d=0}^{\infty} \sum_{n} A_{n, d} Q^{n} q^{d}
$$

where, for each $d$, the inner sum is a Laurent polynomial in $Q$ with integer coefficients $A_{n, d}$. These coefficients $A_{n, d}$ uniquely determine the numbers $n_{d, h}$. But by Theorem 3.20 of [PT], the integrality of the $A_{n, d}$ implies that all of the $n_{d, h}$ are also integers. Thus statement (a) holds.

For $g \geq 2$, the coefficient of $q^{d}$ in (3.5) is a Taylor series in $t^{2}, Z=1+t^{2 g-2} q+O\left(t^{2 g}\right)$, so $\log Z=t^{2 g}-2 q+O\left(t^{2 g}\right)$. Comparing with (3.7) one sees that $n_{d, h}(g)=0$ for all $h<g$, as in (b).

Finally, for genus $g \geq 2$, the inner sum in $(3.8)$ is a Laurent polynomial in $Q$, symmetric in $Q \rightarrow Q^{-1}$, and with degree bounded by $(g-1) \sum h(\square) \leq d^{2}(g-1)$ (since for a partition of $d$, the 
hooklength $h(\square)$ of each box is at most $d)$. This property is preserved under taking the log:

$$
\log Z_{g}^{\text {elem }}=\log \sum_{d} \sum_{n} a_{d, n} Q^{n} q^{d}=\sum_{d \geq 1} \sum_{h \geq g} n_{d, h}(g) \sum_{k \geq 1} \frac{(-1)^{h-1}}{k}\left(Q^{k}+Q^{-k}-2\right)^{h-1} q^{k d},
$$

where $|n| \leq(g-1) d^{2}$. As in the proof of Lemma 3.12 of [PT], this implies the vanishing of $n_{d, h}(g)$ for large $h$. In fact, a proof by induction on $d$ using the above bound implies that $n_{d, h}(g)=0$ for $h-1>d^{2}(g-1)$.

\section{AnAlytic PREliminaries}

This section is a review of the analytic setup for the moduli space for a general closed symplectic manifold $(X, \omega)$. Consider the universal moduli space of simple maps (with smooth, connected domains and smooth $J$ )

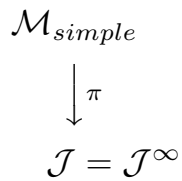

with the projection $\pi([f], J)=J$. Note that $\mathcal{M}_{\text {simple }}$ is an open subset of the universal moduli space $\overline{\mathcal{M}}(X)$ of 1.1 , and simple maps have trivial automorphism groups (cf. [MS], Proposition 2.5.1). It contains the open subset $\mathcal{M}_{e m b}$ of maps that are embeddings.

To set up the analysis, we first work locally around a pair $p=(f, J)$ that represents a point in the moduli space (4.1). Thus $p$ consists of a simple $J$-holomorphic map $f: C \rightarrow X$ whose domain is a smooth, connected marked complex curve $C=\left(\Sigma, x_{1}, \ldots, x_{n}, j\right), J$ is a smooth almost complex structure, and $j$ is in the space $\mathcal{J}(\Sigma)$ of complex structures on $\Sigma$.

4.1. Slice and linearization. The moduli space $\mathcal{M}_{g, n}(X)$ is naturally a subset of the quotient of $\operatorname{Map}(\Sigma, X) \times \mathcal{J}(\Sigma) \times \mathcal{J}$ by the action of the diffeomorphism group $\operatorname{Diff}(\Sigma, \mathbf{x})$ of $\Sigma$ that preserve each point in the set $\mathbf{x}=\left\{x_{1}, \ldots, x_{n}\right\}$ of marked points. In practice, one chooses a local slice for the diffeomorphism action and regards the moduli space locally as a subset of the slice. For now, we assume that the domain $C_{0}$ has no automorphisms; this assumption will be removed at the end of this subsection. To define a slice, choose local holomorphic coordinates on a ball $B \subset \mathcal{M}_{g, n}$ centered at $\left[C_{0}\right] \in \mathcal{M}_{g, n}$. Then there is a local universal deformation $\gamma: \mathcal{U}_{B} \rightarrow B$ of $C_{0}$ with sections $x_{1}, \ldots, x_{n}$. This means, in particular, that the central fiber $\gamma^{-1}(0)$ is identified with $C_{0}$ as a marked complex curve, and every small deformation $C$ of $C_{0}$ is equivalent under $\operatorname{Diff}(\Sigma, \mathbf{x})$ to one and only one fiber $C_{b}, b \in B$, of $\gamma$. Fix a smooth trivialization $\tau$ of $\mathcal{U}_{B} \rightarrow B$ in which the universal deformation is $B \times(\Sigma, \mathbf{x}) \rightarrow B$,

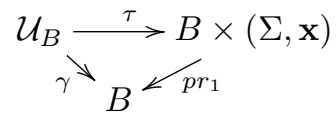

This trivialization, regarded as a family of complex structures $j_{b}$ on $(\Sigma, \mathbf{x})$, defines an embedding

$$
\sigma: B \rightarrow \mathcal{J}(\Sigma), \quad \text { given by } b \mapsto j_{b},
$$

whose image $\mathcal{S}_{\tau}$ is a local slice for the action of the diffeomorphism group on $\mathcal{J}(\Sigma)$. The linearization of this embedding at $C=C_{b}$ gives isomorphisms

$$
T_{C} \mathcal{M}_{g, n} \cong T_{b} B \stackrel{\cong}{\longrightarrow} T_{j_{b}} \mathcal{S}_{\tau} .
$$

Furthermore, the tangent space to the orbit $\mathcal{O}_{j}$ of $\operatorname{Diff}(\Sigma, \mathbf{x})$ on $\mathcal{J}(\Sigma)$ is the image of

$$
\bar{\partial}_{T C}: \Omega_{\mathbf{x}}^{0}(T C) \rightarrow \Omega^{0,1}(T C),
$$


where $\Omega_{\mathbf{x}}^{0}(T C)$ denotes the space of smooth sections of $T C$ that vanish at the marked points.

The slice $\mathcal{S}_{\tau}$ is transverse to this orbit at $j$, giving an isomorphism

$$
T_{j} \mathcal{S}_{\tau} \cong T_{j} \mathcal{J}(\Sigma) / \mathcal{O}_{j}=\Omega^{0,1}(T C) / \operatorname{im} \bar{\partial}_{T C}=H^{0,1}(T C)
$$

where the last equality defines the vector space $H^{0,1}(T C)$. Consequently, the map

$$
D_{C}: \Omega_{\mathbf{x}}^{0}(T C) \oplus T_{j} \mathcal{S}_{\tau} \rightarrow \Omega^{0,1}(T C)
$$

defined by $D_{C}(\zeta, k)=\bar{\partial}_{T C} \zeta+j k$ is a complex-linear isomorphism.

Given local trivializations $\tau_{1}$ and $\tau_{2}$ as in 4.2 over two overlapping charts $B_{1}, B_{2}$ in $\mathcal{M}_{g, n}$ containing $\left[C_{0}\right]$, after restricting them to the overlap $B_{12}=B_{1} \cap B_{2}$, they determine a smooth transition function

$$
\varphi=\tau_{2} \circ \tau_{1}^{-1}: B_{12} \times \Sigma \rightarrow B_{12} \times \Sigma .
$$

The restriction $\varphi_{b}$ to the fiber over $b \in B_{12}$ is a diffeomorphism of $\Sigma$ preserving the marked points $\mathbf{x}$. The corresponding maps 4.3 restrict to embeddings $\sigma_{1}, \sigma_{2}: B_{12} \rightarrow \mathcal{J}(\Sigma)$, and

$$
\sigma_{12}=\sigma_{2} \circ \sigma_{1}^{-1}: \mathcal{S}_{1} \rightarrow \mathcal{S}_{2}
$$

is a diffeomorphism between $\mathcal{S}_{1}=\sigma_{1}\left(B_{12}\right)$ and $\mathcal{S}_{2}=\sigma_{2}\left(B_{12}\right)$ with

$$
\sigma_{12}\left(j_{b}\right)=\left(\varphi_{b}\right)^{*}\left(j_{b}\right) \quad \text { for all } b \in B_{12} \text {. }
$$

To include maps, fix $\left(f_{0}, J_{0}\right)$, where $f_{0}$ is a $J_{0}$-holomorphic map whose domain $C_{0}$ has $\operatorname{Aut}\left(C_{0}\right)=$ 1. Then

$$
\text { Slice }_{\tau}=\left[\operatorname{Map}(\Sigma, X) \times \mathcal{S}_{\tau}\right] \times \mathcal{J}
$$

is a local slice for the action of $\operatorname{Diff}(\Sigma, \mathbf{x})$ on $\operatorname{Map}(\Sigma, X) \times \mathcal{J}(\Sigma) \times \mathcal{J}$. Elements of $4.9 p$ have the form $(f, j, J)$ where $f: \Sigma \rightarrow X$ and $j \in \mathcal{S}_{\tau}$, making $C=\left(\Sigma, j, x_{1}, \ldots, x_{n}\right)$ a marked curve with complex structure $j$. For notational simplicity, we will frequently combine the first two factors, writing elements of 4.9 as pairs $(f, J)$, where the letter $f$ denotes a map $f: C \rightarrow X$ and therefore implicitly includes its domain $C$, regarded as a marked complex curve.

The slice 4.9) comes with a projection

$$
\pi: \text { Slice }_{\tau} \rightarrow \mathcal{J}
$$

defined by $\pi(f, J)=J$, and a complex vector bundle $\mathcal{F} \rightarrow$ Slice $_{\tau}$ whose fiber over $p=(f, J)$ is $\Omega^{0,1}\left(f^{*} T X\right)$. Near $\left(f_{0}, J_{0}\right)$, the moduli space, considered as a subset of $\mathcal{S l i c e}_{\tau}$, is the zero set of the section $\Phi$ of $\mathcal{F}$ defined by

$$
\Phi(f, J)=\bar{\partial}_{J} f .
$$

Under the isomorphism (4.4), the tangent bundle to the slice can be written as

$$
\text { TSlice }_{\tau}=\mathcal{E} \oplus T \mathcal{J},
$$

where $\mathcal{E} \rightarrow$ Slice $_{\tau}$ is the complex bundle whose fiber at $p=(f, J)$ is $\mathcal{E}_{p}=\Omega^{0}\left(f^{*} T X\right) \oplus T_{j} \mathcal{S}_{\tau}$.

The linearization of the $J$-holomorphic map equation 4.10) at a solution $p$ on the slice is the real operator $\mathcal{L}_{p}: \mathcal{E}_{p} \oplus T_{J} \mathcal{J} \rightarrow \mathcal{F}_{p}$ given by

$$
\mathcal{L}_{p}(\xi, K)=D_{p} \xi+\frac{1}{2} K \circ d f \circ j
$$

where $D_{p}: \mathcal{E}_{p} \rightarrow \mathcal{F}_{p}$ is the linearization under variations that fix $J$. Explicitly, $D_{p}$ applied to $\xi=(\zeta, k) \in \Omega^{0}\left(f^{*} T X\right) \oplus T_{j} \mathcal{S}_{\tau}$ is

$$
D_{p}(\xi)(w)=\frac{1}{2}\left[\nabla_{w} \zeta+J \nabla_{j w} \zeta+\left(\nabla_{\zeta} J\right)(d f(j w))+J d f(k(w))\right],
$$

where $\nabla$ is any torsion-free connection on $T X$; at a solution $p, D_{p}$ is independent of the connection (Lemma 2.1.2 [IS1] or Lemma 1.2.1 of [IS2]). Both $\mathcal{L}_{p}$ and $D_{p}$ depend on the $J$ only through the 
1-jet of $J$ along the image of $f$, and the spaces $\mathcal{E}_{p}$ and $\mathcal{F}_{p}$ depend only on the 0 -jet of $J$ along the image of $f$. Furthermore, when the variation $\zeta=f_{*} \zeta^{T}$ is tangent to $C$, (4.13) reduces to

$$
D_{p}\left(f_{*} \zeta^{T}, k\right)=f_{*} D_{C}\left(\zeta^{T}, k\right),
$$

where $D_{C}$ is the isomorphism (4.6). Consequently, when $f: C \rightarrow X$ is a $J$-holomorphic immersion with normal bundle $N_{C}=f^{*} T X / T C$, the linearization (4.13) uniquely descends to a normal operator

$$
D_{p}^{N}: \Gamma\left(N_{C}\right) \rightarrow \Omega^{0,1}\left(N_{C}\right) \text {. }
$$

Now consider a simple map $f_{0}: C_{0} \rightarrow X$ whose automorphism group $\operatorname{Aut}\left(C_{0}\right)$ is non-trivial. As noted after (1.7), the injective points of $f_{0}$ are dense on each component. Hence we can choose $\ell$ additional injective points on $C_{0}$ so that the marked curve $\widetilde{C}_{0}=\left(C_{0}, x_{1}, \ldots, x_{n+\ell}\right)$ has $\operatorname{Aut}\left(\widetilde{C}_{0}\right)=1$. We can then fix a trivialized local universal deformation of the $(n+\ell)$-marked curve $\widetilde{C}_{0}$ over a ball $\widetilde{B} \subset \mathcal{M}_{g, n+\ell}$. Set $\widetilde{\Sigma}=\left(\Sigma, x_{1}, \ldots, x_{n+\ell}\right)$, and for each image point $y_{i}=f_{0}\left(x_{i}\right), n<i \leq n+\ell$, choose a codimension 2 ball $V_{i}$ through $y_{i}$ transverse to $f_{0}\left(C_{0}\right)$. Standard results (cf. Section 3.4 of [MS] show that the space $\operatorname{Map}_{\ell}(\widetilde{\Sigma}, X)$ of maps satisfying $f\left(x_{i}\right) \in V_{i}$ for $n<i \leq n+\ell$ is locally a manifold near $f_{0}$, and hence

$$
\text { Slice }_{\tau}=\operatorname{Map}_{\ell}(\widetilde{\Sigma}, X) \times \widetilde{B} \times \mathcal{J}
$$

is a local slice for the action of $\operatorname{Diff}(\widetilde{\Sigma})$ on $\operatorname{Map}(\widetilde{\Sigma}, X) \times \mathcal{J}(\widetilde{\Sigma}) \times \mathcal{J}$. Thus defined, each point in the slice is a pair $p=(f, J)$ where $f: \widetilde{C} \rightarrow X$ is a map whose domain has no non-trivial automorphisms. The linearization

$$
D_{p}: \mathcal{E}_{p} \rightarrow \mathcal{F}_{p}
$$

is still given by (4.13), but where $\mathcal{E}$ is now the bundle over the slice whose fiber over $p=(f, J)$ is

$$
\mathcal{E}_{p}=\left\{\zeta \in \Omega^{0}\left(f^{*} T X\right) \mid \zeta\left(x_{i}\right) \in T V_{i} \text { for all } n+1 \leq i \leq n+\ell\right\} \oplus T_{\widetilde{C}} \mathcal{M}_{g, n+\ell} .
$$

For notational simplicity, we will henceforth write $\widetilde{C}$ as $C$, and always restrict to sections $\zeta$ with $\zeta\left(x_{i}\right) \in T V_{i}$. All the variations we construct in this and subsequent sections will be supported away from all marked points of $\widetilde{C}$.

4.2. Sobolev completions. Throughout this paper, we work with the following set of Banach space completions (cf. [MS, Section 3.1]). For numbers

$$
l \geq 6, \quad r>2, \quad 1 \leq m \leq l,
$$

let $\mathcal{J}^{l}$ denote the space of tame $C^{l}$ almost complex structures on $X$, let $\operatorname{Map}^{m, r}(\Sigma, X)$ be the completion of the space of smooth maps $\Sigma \rightarrow X$ in the $\operatorname{Sobolev}(m, r)$ norm (i.e. the $m$-jet is in $L^{r}$ ), and let

$$
\text { Slice }_{\tau}^{m, r, l}=\left[\operatorname{Map}^{m, r}(\Sigma, X) \times \mathcal{S}_{\tau}\right] \times \mathcal{J}^{l} .
$$

These are smooth separable Banach manifolds.

Similarly, for each $m$ in the range (4.18), the vector bundles $\mathcal{E}$ and $\mathcal{F}$ extend to vector bundles $\mathcal{E}^{m, r}$ and $\mathcal{F}^{m-1, r}$ over the slice 4.19), whose fibers at $p=(f, J)$ are, respectively,

$$
\mathcal{E}_{p}^{m, r}=W^{m, r}\left(f^{*} T X\right) \oplus T_{j} \mathcal{S}_{\tau} \quad \text { and } \quad \mathcal{F}_{p}^{m-1, r}=W^{m-1, r}\left(\Lambda_{C}^{0,1} \otimes_{\mathbb{C}} f^{*} T X\right),
$$

where $W^{m, r}(E)$ denotes the space of $\operatorname{Sobolev}(m, r)$ sections of a vector bundle $E$, and where $\Lambda_{C}^{0,1}$ is the bundle $\left(T_{\mathbb{C}}^{*} C\right)^{0,1}$ over the domain $C$. The bundle $\mathcal{E}^{m, r}$ is smooth (it is the tangent bundle of $\left.\operatorname{Map}^{m, r}(\Sigma, X) \times S_{\tau}\right)$ and $\mathcal{F}^{m-1, r}$ is of class $C^{l-m}$ (cf. [MS, page 50).

In this context, 4.10 defines a $C^{l-m}$ section of $\mathcal{F}^{m-1, r}$ over the slice 4.19) whose zero locus is a local model of the moduli space. We will focus on the subset

$$
M_{\text {simple }} \subset \mathcal{S l i c e}_{\tau}^{m, r, l}
$$


of pairs $p=(f, J)$ where $f$ is a simple $J$-holomorphic map. By elliptic regularity, all such maps $f$ are of class $W^{l+1, r}$ [MS, Proposition 3.1.10], and hence the set $M_{\text {simple }}$ is independent of $m$ in the range (4.18), and its elements are pairs $(f, J)$ where both $f$ and $J$ are of class $C^{l}$.

For two local trivializations $\tau_{1}, \tau_{2}$ as in 4.2 over the same $B=B_{12}$, there is a transition map $\varphi$ as in (4.7). This induces a map $\widehat{\varphi}$ between two slices 4.9 given by

$$
\widehat{\varphi}\left(f, j_{b}, J\right)=\left(f \circ \varphi_{b},\left(\varphi_{b}\right)^{*}\left(j_{b}\right), J\right)=\left(f \circ \varphi_{b}, \sigma_{12}\left(j_{b}\right), J\right),
$$

for the map $\varphi_{b}$ defined after (4.7) and $\sigma_{12}$ as in (4.8). In this formula, $\sigma_{12}$ is smooth, and $\left\{\varphi_{b} \mid b \in B\right\}$ is a smooth family of diffeomorphisms of the closed surface $\Sigma$. As a result, the regularity of $\widehat{\varphi}$ is determined by the regularity of the map $T$ defined by $T(f, \varphi)=f \circ \varphi$. Formally computing its differential, one finds that

$$
d T_{f, \varphi}(\xi, v)=\varphi^{*} \xi+d f(v), \quad \text { for all } \xi \in \Gamma\left(f^{*} T X\right) \text { and } v \in \Gamma(T C) .
$$

More generally, one finds that the $k$ 'th derivative of $T$ depends on the $k$-jet of $f$. It follows that 4.22 induces a $k$-times differentiable, hence $C^{k-1}$, map

$$
\widehat{\varphi}: \operatorname{Slice}_{\tau_{1}}^{m, r, l} \rightarrow \text { Slice }_{\tau_{2}}^{m-k, r, l} .
$$

Thus a change of trivializations induces a map of slices which loses regularity. We will return to this technical issue in Proposition 5.1 .

4.3. Extensions and adjoints. The linearizations 4.13 extend (non-canonically) to a family of operators parameterized by points $p=(f, J)$ in the slice (4.19) as follows. Fix a Riemannian metric $g_{0}$ on $X$ and let $\nabla^{0}$ denote its Levi-Civita connection. Using the notation of 4.13), define $D_{p}$ by

$$
D_{p}(\zeta, k)=D_{p}^{0} \zeta+\frac{1}{4}(J d f+d f j) k .
$$

where

$$
\left(D_{p}^{0} \zeta\right)(w)=\frac{1}{2}\left(\nabla_{w}^{0} \zeta+J \nabla_{j w}^{0} \zeta\right)+\frac{1}{4}\left(\nabla_{\zeta}^{0} J\right)(d f j+J d f)(w) .
$$

Then $D_{p}^{0}$ agrees with [MS, (3.1.4)], and 4.24 agrees with 4.13) if $f$ is $J$-holomorphic because 4.13) is independent of the connection and $J d f=d f j$. Similarly extend 4.12 by the formula

$$
\mathcal{L}_{p}(\zeta, k)=D_{p} \xi+\frac{1}{4} K(d f j+J d f) .
$$

As in Section 3.1 of [MS,$D_{p}$ and $\mathcal{L}_{p}$ extend to bounded linear operators

$$
D_{p}: \mathcal{E}_{p}^{m, r} \rightarrow \mathcal{F}_{p}^{m-1, r} \quad \text { and } \quad \mathcal{L}_{p}: \mathcal{E}_{p}^{m, r} \oplus T_{J} \mathcal{J}^{l} \rightarrow \mathcal{F}_{p}^{m-1, r},
$$

and $D_{p}$ is a compact perturbation of $D_{p}^{0}$, and hence is Fredholm. Moreover, if $p=(f, J)$ is a $J$-holomorphic pair, then $\operatorname{ker} D_{p}$ and coker $D_{p}$ are independent of $m$ in the range 4.18.

Next fix a Riemannian metric $g_{S}$ compatible with the complex structure on the local universal family of curves parameterized by $\mathcal{S}_{\tau}$. By restriction, $g_{S}$ induces a Riemannian metric on each curve in the local family which, under the trivialization associated with the slice, gives rise to a family of metrics on $\Sigma$ parameterized by the $\mathcal{S}_{\tau}$. These metrics, together with their associated volume forms and the fixed metric $g_{0}$ on $X$ determine $L^{2}$ inner products $\langle,\rangle_{L^{2}}$ on $T_{j} \mathcal{S}_{\tau}, \mathcal{E}_{p}$ and $\mathcal{F}_{p}$ for each $p=(f, J) \in$ Slice $_{\tau}$.

Let $D_{p}^{*}$ denote the formal $L^{2}$ adjoint of the operator $D_{p}$ of 4.24 , which is uniquely defined by

$$
\left\langle D_{p} \xi, \eta\right\rangle_{L^{2}}=\left\langle\xi, D_{p}^{*} \eta\right\rangle_{L^{2}}
$$

for all $\xi \in \Gamma\left(f^{*} T X\right) \oplus T_{j} \mathcal{S}_{\tau}$ and $\eta \in \Omega^{0,1}\left(f^{*} T X\right)$. The adjoint operator depends on the choice of metrics.

Assume $p=(f, J)$ is in a slice 4.19), where $f: C \rightarrow X$ is simple and $J$-holomorphic, and $C$ is a smooth connected complex curve. For an element $\xi=(\zeta, k)$ of $\mathcal{E}_{p}^{m, r}$ and an injective point $x$ of $f$, 
we define $\xi^{N}(x)$ to be the component $\zeta^{N}(x)$ of $\zeta(x)$ normal to $f_{*}\left(T_{x} C\right)$ with respect to the metric $g_{0}$ on $X$.

We will repeatedly use the following simple consequence of elliptic theory.

Lemma 4.1. Fix $p=(f, J)$ in the set $M_{\text {simple }}$ of 4.21. Suppose that $\kappa \in \mathcal{E}_{p}^{0, s}$ and $c \in \mathcal{F}_{p}^{0, s}$, $\frac{1}{s}+\frac{1}{r}=1$, are nonzero weak solutions of $D_{p} \kappa=0$ and $D_{p}^{*} c=0$. Then $\kappa \in \mathcal{E}_{p}^{l, r}$ and $c \in \mathcal{F}_{p}^{l, r}$, and there is an injective point $x \in C$ such that $c(x) \neq 0$ and $\kappa^{N}(x) \neq 0$.

Proof. The equation $D_{p}^{*} c=0$ means that the $L^{2}$ inner product $\left\langle D_{p}(\zeta, k), c\right\rangle_{L^{2}}$ is zero for all $(\zeta, k)$ and therefore, taking $k=0,\left(D_{p}^{0}\right)^{*} c=0$. Lemma 3.4.4 of [MS] then shows that $c$ is in the Sobolev $(l, r)$ space, hence is continuous, and also shows that $c$ cannot vanish identically on any open set in $C$.

Similarly, $\kappa=(\zeta, k)$ is a weak solution of $D_{p}^{0} \zeta=-\frac{1}{2} J d f k$ with $k$ smooth and $f, J$ of class $C^{l}$, and hence $J d f k \in \mathcal{F}^{l-1, r}$. Elliptic regularity as in [MS, Proposition C.2.3] implies that $\zeta$ is in the Sobolev $(l, r)$ space, so $\kappa$ is in $\mathcal{E}^{l, r}$ and is continuous. If $\kappa$ were everywhere tangent to $C$, it would satisfy $D_{C} \kappa=0$ for the operator in 4.6 . But then $\kappa$ would be smooth, and would contradict the fact that (4.6) is an isomorphism. Thus $\kappa^{N} \neq 0$ on some non-empty open set.

The lemma follows because $f$ is at least $C^{2}$ so, by Micallef-White Theorem [MW], the injective points are open and dense in $C$.

\section{The Structure of the MOduli SPACE}

We now consider the completion of the universal moduli space (4.1) in the Sobolev norms introduced in Section 4.2. For simplicity, we will specify the Sobolev norm only when needed. Thus we fix $(l, r)$ as in (4.18) and, without changing notation, let

$$
\begin{gathered}
\mathcal{M}_{\text {simple }} \\
\downarrow^{\pi} \\
\mathcal{J}=\mathcal{J}^{l}
\end{gathered}
$$

be the universal moduli space of equivalence classes $[p]$ (up to reparametrizations of the domain) of pairs $p=(f, J)$, where $J \in \mathcal{J}^{l}$ and $f: C \rightarrow X$ is a simple $J$-holomorphic map of class $W^{l, r}$ whose domain $C$ is a smooth, connected complex curve. This section and the next provide a series of facts about the structure of the moduli space (5.1). These results are proven locally by regarding the moduli space as a subset of a slice 4.19). Proposition 5.1 and Lemma 5.2 hold for any closed symplectic manifold $X$; after that we specialize to Calabi-Yau 6-manifolds, for which the index of $\pi$, given by $(1.3)$, is zero.

5.1. The structure of $\mathcal{M}_{\text {simple }}$. It is well-known that the moduli space (5.1) of simple maps is a manifold. We give a precise statement and proof for later use.

Proposition 5.1. The universal moduli space in (5.1) has the following structure:

(a) The set $M_{\text {simple }} \subset \mathcal{S}$ Sice $e_{\tau}^{m, r, l}$ in (4.21) is a $C^{l-m}$ separable Banach submanifold whose tangent space at $p$ is the kernel of the operator $\mathcal{L}_{p}$ in 4.26 .

(b) For $2 k \leq l-2, \mathcal{M}_{\text {simple }}$ is a $C^{k}$ separable Banach manifold, locally $C^{k}$ diffeomorphic to the subset $M_{\text {simple }}$ of the slice in (a) for each $m$ in the range $1 \leq m \leq l-k$.

In particular, $\mathcal{M}_{\text {simple }}$ is at least $C^{2}$ using Sobolev norms in the range 4.18. 
Proof. (a) As in (4.21), the set $M_{\text {simple }}$ is the zero set of the $C^{l-m}$ section $\Phi$ of $\mathcal{F}^{m-1, r}$ defined by (4.10). By the Implicit Function Theorem, $M_{\text {simple }}$ is a $C^{l-m}$ submanifold of the slice at those points $p$ where $D \Phi_{p}$, which is the operator $\mathcal{L}_{p}$ in (4.26), is onto. By Lemma 4.1, the surjectivity of $\mathcal{L}_{p}$ is independent of $m$ in the range (4.18), so it suffices to consider the case $m=1$. Surjectivity fails at $p=(f, J)$ only if there is a non-zero $c$ in the dual space $\left(\mathcal{F}_{p}^{0, r}\right)^{*}=\mathcal{F}_{p}^{0, s}, s=\frac{r}{r-1}>1$, that is $L^{2}$ orthogonal to $\mathcal{L}_{p}(\xi, K)$ for all $(\xi, K) \in \mathcal{E}_{p}^{1, r} \oplus T_{J} \mathcal{J}^{l}$. By 4.12 and 4.27), this implies that $D_{p}^{*} c=0$ weakly, and

$$
0=\int_{C}\left\langle c, K f_{*} j\right\rangle
$$

for every variation $K$ in $J$. By Lemma 4.1 $c \in \mathcal{F}^{l, r}$ and there is an injective point $x \in C$ where $c(x) \neq 0$. One can then find a variation $K_{0} \in T_{J} \mathcal{J}^{l}$ in $J$ that satisfies $K_{0} f_{*} j=c$ at the point $x$ (cf. [MS, Lemma 3.2.2]). Choose local coordinates $y=\left(y_{1}, y_{2}, \ldots\right)$ on $X$ centered at $f(x)$. Fix a non-negative bump function $\beta(y)$ supported in this coordinate chart and for each $\varepsilon>0$ set $\beta_{\varepsilon}(y)=$ $c(\varepsilon) \beta(y / \varepsilon)$, where $c(\varepsilon)$ is the constant determined by the normalization condition $\int_{C} f^{*} \beta_{\varepsilon}=1$. Then for each continuous function $\varphi$ on $C$ we have

$$
\lim _{\varepsilon \rightarrow 0} \int_{C} f^{*} \beta_{\varepsilon} \cdot \varphi=\varphi(x) .
$$

Substituting $K=\beta_{\varepsilon} K_{0}$ in (5.2) and taking the limit as $\varepsilon \rightarrow 0$ gives a contradiction. Thus

$$
M_{\text {simple }} \subset \text { Slice }_{\tau}^{m, r, l}
$$

is a $C^{l-m}$ submanifold. This can be improved: for any $l \geq m \geq m^{\prime} \geq 1$, the inclusions

$$
M_{\text {simple }} \subset \text { Slice }_{\tau}^{m, r, l} \subset \text { Slice }_{\tau}^{m^{\prime}, r, l}
$$

show that the $C^{l-m}$ atlas obtained from (5.4) can be refined to an $C^{l-m^{\prime}}$ atlas inherited from the enlarged slice (5.5). It follows that for each $k$, the inclusion (5.4) induces a $C^{k}$ atlas on $M_{\text {simple }}$, which is independent of $m$ in the range $1 \leq m \leq l-k$.

(b) The moduli space is covered by images of slices of the form 4.19$)$ under the maps $(f, J) \mapsto$ $([f], J)$, and any two slices with overlapping image are related by a transition map 4.23 . Although (4.23) appears to lose regularity, its restriction to the moduli space does not. Specifically, for any $k \geq 0$ with $l-2 k-1 \geq 1$, the transition map

$$
\widehat{\varphi}: \operatorname{Slice}_{\tau_{1}}^{l-k, r, l} \rightarrow \operatorname{Slice}_{\tau_{2}}^{l-2 k-1, r, l}
$$

is $C^{k}$ (cf. 4.23) ), and maps the $C^{k}$ submanifold $M_{\text {simple }} \subset \mathcal{S}$ Sice $\tau_{1}^{l-k, r, l}$ into the corresponding subset $M_{\text {simple }}^{\prime}$ of $\mathcal{S}$ lice $\tau_{2}^{l-2 k-1, r, l}$ (because reparameterizations of simple maps are simple). The latter inherits a $C^{2 k+1}$ structure from the slice $\mathcal{S}$ lice $e_{\tau_{2}}^{l-2 k-1, r, l}$, and hence a $C^{k}$ structure. Moreover, by the last sentence of part (a), this is the same $C^{k}$ structure that $M_{\text {simple }}^{\prime}$ inherits from its embedding into the slice $\mathcal{S} l i c e_{\tau_{2}}^{l-k, r, l}$. Thus $\widehat{\varphi}$ restricts to a $C^{k}$ bijection from $M_{\text {simple }}$ to $M_{\text {simple }}^{\prime}$; reversing the roles of $\tau_{1}$ and $\tau_{2}$ shows that this is a $C^{k}$ diffeomorphism. This gives a $C^{k}$ atlas on the moduli space. In particular, this applies with $k=[l / 2]-1$, and hence the moduli space has a $C^{2}$-atlas provided $l \geq 6$.

5.2. The wall $\mathcal{W}$. The moduli space $\mathcal{M}_{\text {simple }}$ has a distinguished subset: the "wall" $\mathcal{W} \subset \mathcal{M}_{\text {simple }}$ defined as the stratified set

$$
\mathcal{W}=\bigcup_{s \geq 1} \mathcal{W}^{s}, \quad \mathcal{W}^{s}=\left\{[p] \in \mathcal{M}_{\text {simple }} \mid \operatorname{dim} \operatorname{ker} D_{p}=s\right\}
$$


Lemma 4.1 implies that $\operatorname{ker} D_{p}$ and $\operatorname{ker} D_{p}^{*}$, and hence index $D_{p}$, are independent of the choice of Sobolev norm in the range 4.18. Furthermore, the dimension of $\operatorname{ker} D_{p}$ and of coker $D_{p}$ are preserved under smooth reparametrizations of the domain, so $(5.6)$ is well-defined.

Observe that each $C^{l}$ embedded complex curve $\iota_{C}: C \hookrightarrow X$ determines a set $\mathcal{J}_{C} \subset \mathcal{J}=\mathcal{J}^{l}$ consisting of all $J \in \mathcal{J}$ for which $\iota_{C}$ is $J$-holomorphic. The restriction of 5.1) over $\mathcal{J}_{C}$ has a canonical section $J \mapsto\left(\iota_{C}, J\right)$ whose image is $\mathcal{M}_{C}=\left\{\iota_{C}\right\} \times \mathcal{J}_{C}$.

Lemma 5.2. For each $C^{l}$ embedded complex curve $C, \mathcal{J}_{C}$ is a smooth submanifold of $\mathcal{J}=\mathcal{J}^{l}$.

Proof. Let $j$ denote the complex structure on $C$. Identify $C$ with its image in $X$, and let $E \rightarrow C$ be the vector bundle $E=\operatorname{End}\left(\left.T X\right|_{C}\right)$. At each $x \in C$, the fiber $E_{x}$ of $E$ contains nested submanifolds $E_{x}^{\prime}=\left\{J \in E_{x} \mid J^{2}=-I d.\right\}$ and $E_{x}^{\prime \prime}=\left\{J \in E_{x}^{\prime}|J|_{T_{x} C}=j\right\}$. As $x$ varies, these define $C^{l}$ fiber bundles $E^{\prime}$ and $E^{\prime \prime}$ over $C$. Let $\mathcal{J}^{\prime}$ and $\mathcal{J}^{\prime \prime}$ denote the spaces of $C^{l}$ sections of $E^{\prime}$ and $E^{\prime \prime}$ respectively. By standard theory, $\mathcal{J}^{\prime}$ is a smooth Banach manifold and $\mathcal{J}^{\prime \prime}$ is a submanifold of $\mathcal{J}^{\prime}$. Restricting an almost complex structure $J$ on $X$ to $C$ defines a smooth map

$$
\rho_{C}: \mathcal{J} \rightarrow \mathcal{J}^{\prime}
$$

with $\mathcal{J}_{C}=\rho_{C}^{-1}\left(\mathcal{J}^{\prime \prime}\right)$. The lemma follows if we prove that $\rho_{C}$ is a submersion.

At each $J \in \mathcal{J}_{C}$, the differential of $\rho_{C}$ is simply the restriction $\left(d \rho_{C}\right)_{J}(K)=\left.K\right|_{C}$, and the tangent bundle to $\mathcal{J}^{\prime}$ is the set of all $C^{l}$ sections $Y$ of $E$ that satisfy $J Y+Y J=0$. But every such $Y$ extends to a section $K$ of $T_{J} \mathcal{J}$ : extend $Y$ to a tubular neighborhood of $C$ in $X$, multiply by a smooth cuttoff function to obtain an $\hat{Y}$, and take $K=\frac{1}{2}(\hat{Y}+J \hat{Y} J)$. Thus $\rho_{C}$ is a submersion.

Henceforth (until the end of Section 8) assume that $X$ is a symplectic Calabi-Yau 6-manifold. The results below then show that $\mathcal{W}^{1}$ is a codimension 1 submanifold of $\mathcal{M}_{\text {simple }}$ with a distinguished submanifold $\mathcal{A} \subset \mathcal{W}^{1}$, that the other strata $\mathcal{W}^{r}$ have higher codimension, and that the same is true for the subsets $\mathcal{W}^{r} \cap \mathcal{M}_{C}$ and $\mathcal{A} \cap \mathcal{M}_{C}$ of $\mathcal{M}_{C}$.

Proposition 5.3. Let $X$ be a symplectic Calabi-Yau 6-manifold. Then, as a subset of the universal moduli space in (5.1), the wall has the following structure:

(a) $\mathcal{W}$ is the set of critical points of the projection (5.1), and $\pi$ is a local diffeomorphism on $\mathcal{M}_{\text {simple }} \backslash \mathcal{W}$.

(b) $\mathcal{W}^{1}$ is a codimension 1 submanifold of $\mathcal{M}_{\text {simple }}$.

(c) For each embedded curve $C, \mathcal{M}_{C}=\left\{\iota_{C}\right\} \times \mathcal{J}_{C}$ is transverse to $\mathcal{W}^{1}$.

Proof. (a) In a slice (5.4), the projection $d \pi_{p}(\xi, K)=K$ of a non-zero element in ker $\mathcal{L}_{p}$ is zero if and only if $\xi=(\zeta, k)$ is a non-zero element of ker $D_{p}$. On the other hand, $D_{p}$ has index 0 , so is onto at each $p \notin \mathcal{W}$. At such $p$, for each $K$ we can use 4.12 to obtain $\xi$ with $\mathcal{L}_{p}(\xi, K)=0$; then $(\xi, K)$ is tangent to $\mathcal{M}$ and $d \pi_{p}(\xi, K)=K$. Thus $d \pi$ is an isomorphism at each point not in $\mathcal{W}$, and $\mathcal{W}$ is the collection of critical points of $\pi$.

(b) Fix a representative $p_{0}$ of a point in the wall $\mathcal{W}^{1}$ and fix a slice $\mathcal{S}$ lice $=\mathcal{S}$ lice $e_{\tau}^{1, r, l}$ containing $p_{0}$. Let Fred $\rightarrow \mathcal{S}$ Slice be the fiber bundle whose fiber over $p=(f, J)$ is the space of index 0 Fredholm operators from $\mathcal{E}_{p}=\mathcal{E}_{p}^{1, r}$ to $\mathcal{F}_{p}=\mathcal{F}_{p}^{0, r}$. By choosing a smooth local trivialization of $\mathcal{E}$ and a $C^{l-1}$ local trivialization of $\mathcal{F}$, we can identify Fred with the space of Fredholm operators between two fixed Banach spaces. Then Fred is the union of strata Fred $^{s}=\{D \in$ Fred $\mid \operatorname{dim} \operatorname{ker} D=s\}$, where each Fred ${ }^{s}$ is a submanifold of codimension $s^{2}$ whose normal bundle at $D$ is naturally identified with $\operatorname{Hom}(\operatorname{ker} D, \operatorname{coker} D)(\mathrm{cf}$. $\mathrm{K}, \S 1.1 \mathrm{~b}, \mathrm{c})$. Associating to $p$ the operator 4.26 with $m=1$ defines a section

$$
\Psi(p)=D_{p},
$$

of the $C^{l-1}$ bundle Fred. In fact, $\Psi$ is the vertical derivative of the section $\Phi$ described before 4.21, and hence is of class $C^{l-2}$. On the other hand, $\mathcal{M}_{\text {simple }}$ is $C^{k}$ locally diffeomorphic to the 
submanifold $M_{\text {simple }}$ of the slice for $k$ as in Proposition $5.1 \mathrm{p}$. Noting that $k \leq l-2$, it suffices to show that the restriction of $\Psi$ to $M_{\text {simple }}$ is transverse to Fred ${ }^{1}$.

For this purpose, we consider a deformation $p_{t}=\left(f, J_{t}\right)$ of $p_{0}$ in $M_{\text {simple }}$, where both the map and the complex structure on the domain is fixed, while $J_{t}$ is a path in $\mathcal{J}=\mathcal{J}^{l}$ whose restriction to $f(C)$ is fixed and which changes only in a small neighborhood $U$ of the image $f(x)$ of an injective point $x$. Along the path $p_{t}, \mathcal{E}_{p_{t}}$ is fixed, since it is independent of $J$, as is $\mathcal{F}_{p_{t}}$, which depends on $J$ only through its restriction along $f(C)$. Thus we have a 1-parameter family of Fredholm maps $D_{p_{t}}: \mathcal{E}_{p} \rightarrow \mathcal{F}_{p}$ with fixed domain and target. The initial derivative $\dot{p}_{0}$ has the form $v=(0,0, K) \in \operatorname{ker} \mathcal{L}_{p}=T_{p} M_{\text {simple }}$, where $K$ vanishes along $f(C)$ and is supported in $U$. Hence the variation $\left(\delta_{v} D\right)_{p}=\left.\frac{d}{d t}\right|_{t=0} D_{p_{t}}$ in the direction $v$ is obtained by replacing $J$ by $J_{t}$ in 4.13 and taking the $t$-derivative at $t=0$. Because the formula 4.13 is independent of the connection, we can take the variation with the connection fixed; the formula then shows that $\left(\delta_{v} D\right)_{p}$ is $\frac{1}{2}(\nabla K) f_{*} j$. Moreover, when applied to an element $\xi=(\zeta, k)$ of $\mathcal{E}_{p}$, this variation depends only on the normal component $\xi^{N}=\left(\zeta^{N}, 0\right)$ of $\zeta$ because $K \equiv 0$ on $f(C)$ :

$$
\left(\delta_{v} D\right)_{p} \xi=\frac{1}{2}\left(\nabla_{\xi^{N}} K\right) f_{*} j .
$$

This formula is tensorial in $\xi$, does not depend on the connection, and is tensorial in $K \in T_{J} \mathcal{J}$ as long as $K \equiv 0$ along $f(C)$.

Now fix generators $\kappa$ of $\operatorname{ker} D_{p}$ and $c$ of $\operatorname{ker} D_{p}^{*}$; these are continuous by Lemma 4.1 . Since the normal space to Fred ${ }^{1}$ at $p$ is 1 dimensional, it is enough to construct a variation in $p$ of the form $(0,0, K)$ such that the $L^{2}$ inner product $\left\langle c,\left(\delta_{v} D\right)_{p} \kappa\right\rangle_{L^{2}}$ is non-zero.

To find such a $v$, choose an injective point $x \in C$ of $f$ with both $\kappa^{N}(x) \neq 0$ and $c(x) \neq 0$ as in Lemma 4.1. As in the proof of Proposition 5.1p, there is a $K_{0} \in T_{J} \mathcal{J}^{l}$ supported near $f(x)$ such that $K_{0} d f j=c$ at the point $x$. Because $f$ is $C^{l}$, there is a neighborhood of $x$ in $C$ whose image under $f$ is an embedded $C^{l}$ submanifold of $X$. Hence we can choose a local $C^{l}$ coordinate system $\left\{z, y_{1}, y_{2}, \ldots\right\}$ centered at $f(x)$ with $z$ a local complex coordinate on $f(C)$, and $\left\{y_{i}\right\}$ real coordinates vanishing along $f(C)$, and such that $\left.\frac{\partial}{\partial y_{1}}\right|_{f(x)}=\kappa^{N}(x)$. Then $K=y_{1} K_{0}$ lies in $T_{J} \mathcal{J}^{l}$, vanishes along $f(C)$, and satisfies

$$
\left(\nabla_{\kappa^{N}} K\right) f_{*} j=c \text { at the single point } x .
$$

Finally, set $v_{\varepsilon}=\left(0,0,2 \beta_{\varepsilon} K\right)$ with $\beta_{\varepsilon}$ as in (5.3). Replacing $K$ by $2 \beta_{\varepsilon} K$ in 5.8 ) and using (5.9), one sees that

$$
\lim _{\varepsilon \rightarrow 0} \int_{C}\left\langle c,\left(\delta_{v_{\varepsilon}} D\right)_{p} \kappa\right\rangle=|c(x)|^{2} \neq 0 .
$$

In particular, there is a variation with $\left\langle c,\left(\delta_{v} D\right)_{p} \kappa\right\rangle_{L^{2}} \neq 0$, which proves statement (c).

Statement (c) holds because the transversality above was obtained using a variation $v_{\varepsilon}=\left(0,2 \beta_{\varepsilon} K\right)$ tangent to $\mathcal{M}_{C}$.

5.3. The structure of $\mathcal{W} \backslash \mathcal{W}^{1}$. The proof of Proposition $5.3 \mathrm{~b}$ extends to show that the part of $\mathcal{W}$ not in $\mathcal{W}^{1}$ has codimension 3 in the following sense.

Definition 5.4. We say that a subset $S$ of a manifold $M$ has codimension $k$ if it is contained in a countable union $\bigcup \rho_{\ell}\left(S^{\ell}\right)$, where each $\rho_{\ell}: S^{\ell} \rightarrow M$ is a Fredholm map of separable Banach manifolds with index $\rho_{\ell} \leq-k$.

Lemma 5.5. $\mathcal{W} \backslash \mathcal{W}^{1}$ has codimension 3 in $\mathcal{M}_{\text {emb }}$, and $\left(\mathcal{W} \backslash \mathcal{W}^{1}\right) \cap \mathcal{M}_{C}$ has codimension 3 in $\mathcal{M}_{C}$.

C. Taubes obtained a similar result in dimension 4 using analytic perturbation theory (Step 5 of the proof of Lemma $5.1 \mathrm{in}[\mathrm{T}$ ). A proof in the spirit of the above arguments is given in the second appendix as Proposition B.6. Lemma 5.5 is a special case. 
5.4. The structure of $\mathcal{W}^{1}$. We next examine the portion of the top stratum $\mathcal{W}^{1}$ of the wall (5.6) that lies in the open subset $\mathcal{M}_{e m b}$ of $\mathcal{M}_{\text {simple }}$ consisting of embedded maps. Our goal is to show that the projection

$$
\pi_{\mathcal{W}}: \mathcal{W}^{1} \rightarrow \mathcal{J}
$$

obtained by restricting (5.1) to $\mathcal{W}^{1}$ is an immersion off a set of codimension 1. For this purpose, we first introduce locally defined functions $\psi$ that vanish transversally along $\mathcal{W}^{1}$.

Fix a slice Slice containing a representative $p_{0}$ of a point in $\mathcal{W}^{1}$, regard $\mathcal{W}^{1}$ locally as a subset of this slice, and consider the vector bundles $\mathcal{E}=\mathcal{E}^{1, r}$ and $\mathcal{F}=\mathcal{F}^{0, r}$ on the slice. At every $p \in \mathcal{W}^{1}$, the operators $D_{p}: \mathcal{E}_{p} \rightarrow \mathcal{F}_{p}$ and $D_{p}^{*}: \mathcal{F}_{p}^{1, r} \rightarrow \mathcal{E}_{p}^{0, r}$ defined by 4.24 and 4.27) have 1-dimensional kernels. These kernels determine subbundles

$$
\mathcal{E}^{0} \subset \mathcal{E}, \quad \mathcal{F}^{0} \subset \mathcal{F}
$$

along $\mathcal{W}^{1}$, and the projection $\pi_{c o k}: \mathcal{F}^{0} \rightarrow \mathcal{F} /$ im $D$ onto the cokernel bundle along $\mathcal{W}^{1}$ is an isomorphism. By choosing sections of $\mathcal{E}^{0}$ and $\mathcal{F}^{0}$ along the submanifold $\mathcal{W}^{1}$ and extending, we can find non-vanishing $C^{2}$ local sections $\kappa$ of $\mathcal{E}$ and $c$ of $\mathcal{F}$, defined in a neighborhood $U$ of $p_{0}$ in the slice, such that the restrictions to $\mathcal{W}^{1}$ are local sections of $\mathcal{E}^{0}$ and $\mathcal{F}^{0}$ respectively. Let $\psi: U \rightarrow \mathbb{R}$ be the function defined by

$$
\psi(q)=\int_{C}\left\langle c_{q}, D_{q} \kappa_{q}\right\rangle,
$$

using the same metrics and volume forms as in 4.27).

Clearly, $\psi$ vanishes along $\mathcal{W}^{1} \cap U$, where $D_{q} \kappa_{q}=0$. Differentiating $\psi\left(q_{t}\right)$ for any path $q_{t}$ in $U$ with $q_{0}=p \in \mathcal{W}^{1} \cap U$ and initial velocity $\dot{q}_{0}=u$ yields several terms, including the variation in the inner product and volume form. All except one vanish at $q_{0}=p$ because $D_{p} \kappa_{p}=0$ and $D_{p}^{*} c_{p}=0$, showing that

$$
(d \psi)_{p}(u)=\int_{C}\left\langle c_{p},\left(\delta_{u} D\right)_{p} \kappa_{p}\right\rangle .
$$

The proof of Proposition $5.3 \mathrm{~b}$ produces variations showing that $(d \psi)_{p} \neq 0$ for all $p \in \mathcal{W}^{1} \cap U$. Thus the restriction of $\psi$ to $\mathcal{M}_{\text {simple }} \cap U$ vanishes transversally along $\mathcal{W}^{1} \cap U$. In particular, we have

$$
T_{p} \mathcal{W}^{1}=(\operatorname{ker} d \psi)_{p} \quad \forall p \in \mathcal{W}^{1} \cap U .
$$

Lemma 5.6. Inside $\mathcal{M}_{\text {emb }}$, the subset $\mathcal{A}$ of $\mathcal{W}^{1}$ where the projection (5.11) fails to be a immersion is a codimension 1 submanifold of $\mathcal{W}^{1}$, and $\mathcal{M}_{C}$ is transverse to $\mathcal{A}$.

Proof. Fix a point in $\mathcal{A}$ and a slice containing it, and work locally in a neighborhood $U$ on which $\psi$ is defined by (5.13). Consider the vector field $v=(\kappa, 0)$ on $U$, where $\kappa$ is the non-vanishing local section chosen above (5.13). The proof of Proposition 5.3 a shows that at every $p \in \mathcal{W}^{1}, \operatorname{ker}(d \pi)_{p}$ is spanned by $v_{p}$, so $\pi_{\mathcal{W}}$ fails to be an immersion at $p$ if and only if $v_{p} \in T_{p} \mathcal{W}^{1}$. Together with (5.14), this gives two local descriptions of $\mathcal{A}$ :

$$
\mathcal{A}=\left\{p \in \mathcal{W}^{1} \mid(\kappa, 0)_{p} \in T_{p} \mathcal{W}^{1}\right\}=\left\{p \in \mathcal{W}^{1} \mid(d \psi(v))_{p}=0\right\} .
$$

By the second description, it suffices to show that the restriction of the function $d \psi(v): U \rightarrow \mathbb{R}$ to $\mathcal{W}^{1}$ vanishes transversally at each $p \in \mathcal{A}$.

Consider variations in $p=(f, J)$ of the form $w=(0, K)$, where $K$ is an element of $T_{J} \mathcal{J}^{l}$ such that both $K=0$ and $\nabla K=0$ along the image $f(C)$. We will show that for every such variation

$$
w=(0, K) \in T_{p} \mathcal{W}^{1}
$$

and

$$
\left.\nabla_{w}(d \psi(v))\right|_{p}=\frac{1}{2} \int_{C}\left\langle c,\left(\nabla_{\kappa^{N}} \nabla_{\kappa^{N}} K\right) d f j\right\rangle .
$$


The argument used to obtain $(5.9)$ and 5.10 then produces a $K=\frac{1}{2} y_{1}^{2} \beta_{\varepsilon} K_{0}$ in $T_{J} \mathcal{J}^{l}$ with $K=0$ and $\nabla K=0$ along $f(C)$ that makes the integral (5.17) non-zero, which shows transversality at $p$. Furthermore, if $p \in \mathcal{M}_{C}$, these variations $w=(0, K)$ are tangent to $\mathcal{M}_{C}$. Thus both parts of the lemma follow from 5.16 and (5.17).

We will prove (5.16) and (5.17) by constructing a 2-parameter family of deformations of $p$ in $U$ that is tangent to $v$ and $w$ at $p$. For clarity, write $p$ as $p_{0}=\left(f_{0}, J_{0}\right)$. Start by choosing a path $J_{t}$ through $J_{0}$ with initial velocity $K \in T_{J_{0}} \mathcal{J}^{l}$ such that the restrictions of $J_{t}$ and $\nabla^{0} J_{t}$ to $f_{0}(C)$ are independent of $t$. Along the path $q_{t}=\left(f_{0}, J_{t}\right)$ in $U, \mathcal{E}_{q_{t}}, \mathcal{F}_{q_{t}}$ and the operators $D_{q_{t}}$ defined by (4.24) are constant, because all three depend only on the 1-jet of $J_{t}$ along $f_{0}(C)$, which is fixed. Thus the path $\left(f_{0}, J_{t}\right)$ is in $\mathcal{W}^{1}$ for all $t$, so its initial tangent $w$ satisfies (5.16). We can assume that the local sections $\kappa$ of $\mathcal{E}$ and $c$ of $\mathcal{F}$ used to define $\psi$ were chosen so that $\kappa=\kappa_{0}$ and $c=c_{0}$ along the path $\left(f_{0}, J_{t}\right)$.

Next, choose a smooth family of maps $f_{s}:\left(C, j_{s}\right) \rightarrow X$ with initial tangent vector $\kappa_{0}$. Then

$$
p_{s, t}=\left(f_{s}, J_{t}\right)
$$

is a 2-parameter family in the slice with $p_{*} \partial_{s}=\left(\kappa_{0}, 0\right)=v$ along $p_{0, t}$ and $p_{*} \partial_{t}=(0, K)=w$ at $s=t=0$. Write the restrictions of the sections $\kappa, c$ and the operators 4.24 as $\kappa_{s, t}, c_{s, t}$ and $D_{s, t}$ respectively, and set

$$
\eta_{s, t}=D_{s, t} \kappa_{s, t}
$$

For $s=0, \eta_{0, t}=D_{f_{0}, J_{t}} \kappa_{0}$ is 0 by construction; we also have $c_{0, t}=c_{0}, \kappa_{0, t}=\kappa_{0}$, and therefore

$$
\eta_{0,0}=0, \quad\left(\partial_{t} \eta\right)_{0,0}=0, \quad\left(\partial_{t} c\right)_{0,0}=0, \quad\left(\partial_{t} \kappa\right)_{0,0}=0
$$

With this notation, the restriction of 5.13 to the family 5.18 is the function

$$
\psi(s, t)=\psi\left(p_{s, t}\right)=\int_{C}\left\langle c_{s, t}, D_{s, t} \kappa_{s, t}\right\rangle_{s}=\int_{C}\left\langle c_{s, t}, \eta_{s, t}\right\rangle_{s},
$$

where the pointwise inner product and the area form depend on $s$ but not on $t$. Along the path $p_{0, t}$, we have $d \psi(v)=d \psi\left(p_{*} \partial_{s}\right)=\partial_{s} \psi$. Differentiating in the $w$ direction and noting that $w=p_{*} \partial_{t}$ at the origin then gives

$$
\nabla_{w}(d \psi(v))_{p_{0}}=\left(\partial_{t} \partial_{s} \psi\right)_{0,0}=\left(\partial_{s} \partial_{t} \psi\right)_{0,0}
$$

To complete the proof, we will calculate $\left(\partial_{s} \partial_{t} \psi\right)_{0,0}$ by differentiating (5.21). For fixed $s, \kappa_{s, t}$ is a path of sections of the fixed bundle $f_{s}^{*} T X$, while both $c_{s, t}$ and $\eta_{s, t}$ are paths of 1 -forms on $C$ with values in the fixed bundle $f_{s}^{*} T X$ (they are $(0,1)$ forms with respect to the pair $\left(j_{s}, J_{t}\right)$ ). Hence we have

$$
\left(\partial_{t} \psi\right)_{s, 0}=\int_{C}\left\langle\left(\partial_{t} c\right)_{s, 0}, \eta_{s, 0}\right\rangle_{s}+\int_{C}\left\langle c_{s, 0},\left(\partial_{t} \eta\right)_{s, 0}\right\rangle_{s} .
$$

Now differentiate 5.22 with respect to $s$ and evaluate at $s=0$ using 5.20 . The contribution of the first integral vanishes because $\left(\partial_{t} c\right)_{0,0}=0$ and $\eta_{0,0}=0$, leaving

$$
\left(\partial_{s} \partial_{t} \psi\right)_{0,0}=\left.\partial_{s}\right|_{s=0} \int_{C}\left\langle c_{s, 0},\left(\partial_{t} \eta\right)_{s, 0}\right\rangle_{s} .
$$

By (5.19), $\eta_{s, t}$ is given by the operator 4.24 with $q=\left(f_{s}, J_{t}\right)$, applied to $\kappa_{s, t}=\left(\zeta_{s, t}, k_{s, t}\right)$. In the resulting formula, fix $s$ and differentiate with respect to $t$. Because $f_{s}$ and $j_{s}$ are independent of $t$ and $\left(\partial_{t} J_{t}\right)_{0}=K$, one sees that $\left(\partial_{t} \eta\right)_{s, 0}$ has the general form

$$
\left(\partial_{t} \eta\right)_{s, 0}=D_{s, 0}\left(\left(\partial_{t} \kappa\right)_{s, 0}\right)+\frac{1}{4}\left(\nabla_{\zeta_{s}}^{0} K\right)\left(d f_{s} j_{s}+J_{0} d f_{s}\right)+T_{s, t}(K)
$$


where $T(K)$ is a sum of terms, each linear and tensorial in $K$. The contribution of the first term of (5.24) to (5.23) is

$$
\left.\partial_{s}\right|_{s=0} \int_{C}\left\langle c_{s, 0}, D_{s, 0}\left(\left(\partial_{t} \kappa\right)_{s, 0}\right)\right\rangle_{s}=\left.\partial_{s}\right|_{s=0} \int_{C}\left\langle D_{s, 0}^{*} c_{s, 0},\left(\partial_{t} \kappa\right)_{s, 0}\right\rangle_{s},
$$

as in (4.27). Taking the $s$-derivative at $s=0$ yields three terms, all of which vanish because $D_{0,0}^{*} c_{0,0}=0$ and $\left(\partial_{t} \kappa\right)_{0,0}=0$. Similarly inserting the remaining terms of 5.24 into 5.23 and differentiating yields many terms; all but one vanish because $K$ and $\nabla^{0} K$ vanish along $f_{0}(C)$. After noting that $f_{0}$ is $J_{0}$-holomorphic, one is left with

$$
\left(\partial_{s} \partial_{t} \psi\right)_{0,0}=\frac{1}{2} \int_{C}\left\langle c_{0}, \nabla_{\zeta_{0}}^{0} \nabla_{\zeta_{0}}^{0} K d f_{0} j_{0}\right\rangle_{0} .
$$

Because $K$ and $\nabla K$ vanish along $f_{0}(C)$, this expression is independent of the connection, and its dependence on $\zeta_{0}$ involves only the normal component $\zeta_{0}^{N}=\kappa_{0}^{N}$. Thus

$$
\left.\nabla_{w}(d \psi(v))\right|_{p_{0}}=\left(\partial_{s} \partial_{t} \psi\right)_{0,0}=\frac{1}{2} \int_{C}\left\langle c_{0}, \nabla_{\kappa_{0}^{N}} \nabla_{\kappa_{0}^{N}} K d f_{0} j_{0}\right\rangle_{0} .
$$

This verifies (5.17) and completes the proof.

\section{LOCAL MODELS FOR WALL CROSSINGS}

We next study the local geometry of the moduli space around a point $p$ on the wall $\mathcal{W}^{1} \backslash \mathcal{A}$. We assume that $p$ corresponds to a $J$-holomorphic embedding $f: C \rightarrow X$ of a smooth curve $C$, which we can regard as the inclusion $\iota_{C}: C \hookrightarrow X$ of its image. The goal is to show that the restriction $\pi_{\gamma}: \mathcal{M}_{e m b}^{\gamma} \rightarrow \gamma$ of (5.1) over a generic path $\gamma \subset \mathcal{J}$ is a Morse function at $p$, and hence is locally described by a quadratic equation. Two types of smooth paths in $\mathcal{J}=\mathcal{J}^{l}$ passing through $J$ are relevant for our purposes:

TYPE A. $\gamma_{A}$ is a path in $\mathcal{J}$ such that the projection $\pi$ is transverse to $\gamma_{A}$ at $p$.

Type B. $\gamma_{B}$ is a path in $\mathcal{J}_{C}$ whose lift $\widetilde{\gamma}_{B}=\left\{\iota_{C}\right\} \times \gamma_{B}$ to $\mathcal{M}_{C}$ is transverse to $\mathcal{W}^{1}$ at $p$.

The lemmas at the end of this section show that both types of paths are generic. But first, we will use Kuranishi's method to construct a local model for the moduli space over these paths. It is convenient to study both types simultaneously by considering embedded parameterized disks

$$
S=\{(t, s)\} \subset \mathcal{J}
$$

whose $t$-axis is a path $\gamma_{B}$ of Type B and whose $s$-axis is a path $\gamma_{A}$ of Type A. We then restrict 5.1) over $S$ to obtain

$$
\pi_{S}: \mathcal{M}^{S} \rightarrow S
$$

where $\mathcal{M}^{S}=\pi^{-1}(S)$ is the moduli space over $S$. As in Section 5 , we regard $p$ as a point in a slice Slice of the form 4.19 with $m=1$ and, without changing notation, locally identify $\mathcal{M}_{e m b}$ with the corresponding submanifold of the slice.

Definition 6.1. A 3-ball $B \subset$ Slice with coordinates $(x, y, z)$ centered at $p=(f, J) \in \mathcal{W}^{1} \backslash \mathcal{A}$ is adapted to $S$ at $p$ if

(a) $\pi: B \rightarrow S$ is given by $\pi(x, y, z)=(y, z)$.

(b) $\gamma_{B}(t)=(t, 0)$ is a Type $B$ path whose lift is $\tilde{\gamma}_{B}(t)=(0, t, 0)$.

(c) $\gamma_{A}(s)=(0, s)$ is a Type A path. 
(d) In terms of the splitting (4.11), $T_{p} B$ is spanned by $\left.\partial_{x}\right|_{p}=(\kappa, 0),\left.\partial_{y}\right|_{p}=\left(0, K_{B}\right)$, and $\left.\partial_{z}\right|_{p}=$ $\left(0, K_{A}\right)$, where $\kappa$ generates $\operatorname{ker} D_{p} \cong \mathbb{R}, K_{A}=\dot{\gamma}_{A}(0)$ and $K_{B}=\dot{\gamma}_{B}(0)$.

With these assumptions, the transversality conditions in Type A and B are equivalent to

$$
\text { (a) } K_{A} \notin \operatorname{im~} d \pi_{p} \quad \text { and } \quad(b) \quad\left(0, K_{B}\right) \notin T_{p} \mathcal{W}^{1}
$$

respectively. The requirement that $\gamma_{B} \subset \mathcal{J}_{C}$ also implies that $K_{B}$ vanishes along $f(C)$, so $\left(0, K_{B}\right) \in$ $\operatorname{ker} \mathcal{L}_{p}=T_{p} \mathcal{M}_{e m b}$; because $d \pi_{p}(0, K)=K$ this also means that $K_{B} \in \operatorname{im} d \pi_{p}$ and hence $K_{A}$ and $K_{B}$ are linearly independent.

Theorem 6.2 (Kuranishi model). For each $p=(f, J)$ in $\mathcal{M}_{\text {emb }} \cap\left(\mathcal{W}^{1} \backslash \mathcal{A}\right)$ and each $S \subset \mathcal{J}$ as in (6.1) centered at $J$, there is a 3-ball $B$ adapted to $S$ at $p$ such that $\mathcal{M}^{S}$ is locally the 2-manifold

$$
V=\{(x, y, z) \in B \mid z=x(a x+b y+r(x, y))\}
$$

with $a, b \neq 0$, where $r(x, y)=O\left(x^{2}+y^{2}\right)$ near the origin. Moreover $\mathcal{W}^{1} \cap \mathcal{M}^{S}$ is locally modeled on the zero locus of the function $w: \mathcal{M}^{S} \rightarrow \mathbb{R}$ given by

$$
w(x, y)=z_{x}(x, y)=2 a x+b y+(x r(x, y))_{x} .
$$

The tangent space $T_{p}\left(\mathcal{W}^{1} \cap \mathcal{M}^{S}\right)$ is the intersection of the kernels of the 1-forms $d z$ and

$$
d w=2 a d x+b d y
$$

at the origin.

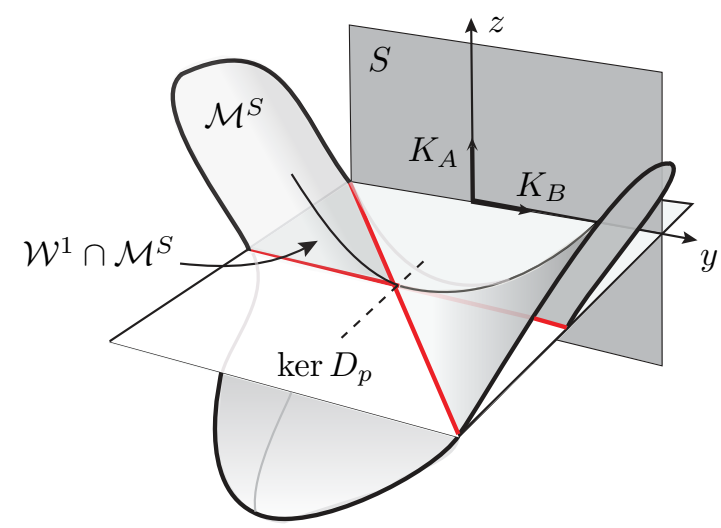

Figure 2. In the local model, $\mathcal{M}^{S}$ is a saddle and $\pi: \mathcal{M}^{S} \rightarrow S$ is the projection onto the $y z$-plane (at the back). At the origin, $T_{p} \mathcal{M}^{S}$ is spanned by $\partial_{x} \subset \operatorname{ker} D_{p}$ and $\partial_{y}=\left(0, K_{B}\right)$, while $\partial_{z}=\left(0, K_{A}\right)$ is normal to $\mathcal{M}^{S}$.

Theorem 6.2 shows that $\mathcal{M}^{S}$ is locally a saddle surface in $B \subset \mathbb{R}^{3}$ given as the graph of a function $z=z(x, y)$ that has a non-degenerate critical point at the origin. Before giving the proof, we record the two cases that will be used in later sections.

Corollary 6.3. Suppose that $p=(f, J) \in \mathcal{M}_{\text {emb }} \cap\left(\mathcal{W}^{1} \backslash \mathcal{A}\right)$ and $\gamma$ is a path in $\mathcal{J}$ through $\gamma(0)=J$.

(a) If $\gamma$ is of Type $A$, then $\mathcal{M}^{\gamma}$ is locally modeled at $p$ by

$$
\left\{(x, t) \mid t=a x^{2}\right\},
$$

with $a \neq 0$ and $\pi_{\gamma}(x, t)=t$.

(b) If $\gamma$ is of Type $B$, then there is a disk $S \subset \mathcal{J}$ centered at $p$ locally containing $\gamma$ such that $\mathcal{M}^{S}$ is locally modeled at $p$ by

$$
\{(x, t, s) \in B \mid s=x(a x+b t+r(x, t))\},
$$

with $a, b \neq 0, r(x, t)=O\left(x^{2}+t^{2}\right)$, and $\pi_{S}(x, t, s)=(t, s)$, and such that the restriction to $\mathcal{M}^{\gamma}$ is the restriction to the plane $s=0$, namely

$$
\{(x, t) \mid 0=x(a x+b t+r(x, t))\} \text {. }
$$


Proof. In the first case, take $\gamma_{A}=\gamma$, choose $\gamma_{B} \subset \mathcal{J}_{C}$ a path whose tangent vector $K_{B}$ satisfies (6.3 ), and choose a local embedded disk $S \subset \mathcal{J}$ containing both $\gamma_{A}$ and $\gamma_{B}$ locally near $p$. Then apply Theorem 6.2 and restrict to the plane $\{y=0\}$ to get the local model for $\mathcal{M}^{\gamma}$ of the form

$$
\{(x, t) \mid t=x(a x+r(x))\} \quad \text { with } a \neq 0, \pi_{\gamma}(x, t)=t,
$$

and with $r(x)=O\left(x^{2}\right)$ for small $x$. This becomes 6.5 after reparameterizing $x$.

Similarly, in the second case, take $\gamma_{B}=\gamma$, fix a direction $K_{A} \in T_{J} \mathcal{J}$ satisfying $(6.3$ a), choose $S$ with $T_{J} S=\operatorname{span}\left(K_{A}, K_{B}\right)$ containing $\gamma$, and again apply Theorem 6.2 .

The distinction between the local models 6.5) and 6.7) is crucial. By Lemma 6.5 below, Model 6.5 applies where a generic path in $\mathcal{J}$ crosses a wall. This is precisely the local model for the creation (if $a>0$ ) or annihilation (if $a<0$ ) of a pair of curves in the moduli space. Similarly, Lemma 6.7 shows that Model (6.7) applies where a generic path in the subspace $\mathcal{J}_{C} \subset \mathcal{J}$ crosses a wall. But the model (6.7) is not a manifold: it is the union of two curves crossing transversely at the origin. It can be smoothed using the parameter $s$ in $(6.6)$, as will be done in Lemma 7.4

Proof of Theorem 6.2. Specializing (4.19) and (4.11), one sees that the slice over $S$ is $\operatorname{Map}^{1, r}(\Sigma, X) \times$ $\mathcal{S}_{\tau} \times S$ and that we can identify the first two factors with their tangent space at $p$, which is $\mathcal{E}_{p}=\mathcal{E}_{p}^{1, r}$. We can also trivialize the bundle $\mathcal{F}=\mathcal{F}^{0, r}$ over a neighborhood of $p$. With these identifications, $\mathcal{M}^{S}$ is the subset of $\mathcal{E}_{p} \times S$ that is the zero set of the $\mathcal{F}_{p}$-valued $C^{l-1}$ function $F$ defined by 4.10]. This has an expansion

$$
F(\xi)=\mathcal{L}_{p}(\xi)+Q(\xi),
$$

where $Q(\xi)$ vanishes to first order at $\xi=0$. Next, fix a generator $\kappa$ of $\operatorname{ker} D_{p}=\mathcal{E}^{0}$ and choose a decompositon $\mathcal{E}_{p}=\mathcal{E}^{0} \oplus \mathcal{E}^{+}$. Then $\xi$ can be written as $\xi=(x, y, z, \alpha)$, for coordinates $(x, y, z)$ in a 3 -ball $\mathcal{B} \subset \mathcal{E}^{0} \times S$ around 0 and $\alpha \in \mathcal{E}^{+}$. Using (4.12), the linear term is

$$
\begin{aligned}
\mathcal{L}_{p}(\xi) & =x D_{p} \kappa+\frac{1}{2}\left(y K_{B}+z K_{A}\right) f_{*} j+D_{p} \alpha \\
& =z c_{p}+D_{p} \alpha,
\end{aligned}
$$

where we have set $c_{p}=\frac{1}{2} K_{A} f_{*} j$ and noted that $K_{B}=\dot{\gamma}_{B}(0)$ is tangent to a Type B path, so $\left.K_{B}\right|_{f(C)}=0$. Furthermore, the transversality assumption $(6.3 \mathrm{a})$ ensures that $c_{p} \notin \operatorname{im} D_{p}$ as follows: if $c_{p}=D_{p} \mu$, then $\left(-\mu, K_{A}\right)$ is an element of ker $\mathcal{L}_{p}=T_{p} \mathcal{M}_{\text {simple }}$ with $d \pi\left(-\mu, K_{A}\right)=K_{A}$, contradicting (6.3). Thus $\mathcal{F}_{p}$ decomposes as

$$
\mathcal{F}_{p}=\widehat{\mathcal{F}}^{0} \oplus \widehat{\mathcal{F}}^{+}
$$

where $\widehat{\mathcal{F}}^{0}$ is the real span of $c_{p}$ and $\widehat{\mathcal{F}}^{+}=D_{p} \mathcal{E}^{+}$. Using this decomposition, write $Q$ as $\left(Q_{0} \cdot c_{p}, Q_{1}\right)$.

By Proposition A.4.1 of [MS] there is a bounded linear map $T: \widehat{\mathcal{F}}^{+} \rightarrow \mathcal{E}^{+}$that is a pseudo-inverse of $D_{p}$, which implies that $D_{p} T$ is the identity on $\widehat{\mathcal{F}}^{+}$. Define a map

$$
\eta: \mathcal{E}^{0} \times S \times \mathcal{E}^{+} \rightarrow \mathcal{E}^{0} \times S \times \mathcal{E}^{+}
$$

by

$$
\eta(x, y, z, \alpha)=\left(x, y, z, \eta_{x, y, z}(\alpha)\right), \quad \text { where } \quad \eta_{x, y, z}(\alpha)=\alpha+T Q_{1}(x, y, z, \alpha) \in \mathcal{E}^{+} .
$$

By the Inverse Function Theorem, $\eta$ is a local diffeomorphism near 0.

Using the above notation, 6.8 can be rewritten as the equation

$$
F(x, y, z, \alpha)=\left(\left(z+Q_{0}\right) c_{p}, D_{p}\left(\eta_{x, y, z}(\alpha)\right)\right) \in \widehat{\mathcal{F}}^{0} \oplus \widehat{\mathcal{F}}^{+} .
$$

This shows that $F(x, y, z, \alpha)=0$ if and only if both $\eta_{x, y, z}(\alpha)=T D_{p}\left(\eta_{x, y, z}(\alpha)\right)=0$, and $z=q$ where

$$
q(x, y, z)=-Q_{0} \circ \eta^{-1}(x, y, z, 0) .
$$


Thus there is a local diffeomorphism

$$
\mathcal{M}^{S} \cong\{(x, y, z) \in B \mid z=q(x, y, z)\} .
$$

The real-valued function $q$ is smooth and vanishes to first order at the origin, so we can solve for $z$ as a function of $x$ and $y$ to obtain

$$
z=z(x, y)=a x^{2}+b x y+c y^{2}+r_{2}(x, y),
$$

where the remainder $r_{2}$ vanishes to second order. In particular, locally near $p, \mathcal{M}^{S}$ is a 2-manifold with coordinates $(x, y)$, in which the projection $(6.2)$ is

$$
\pi_{S}(x, y)=(y, z(x, y))
$$

so $d \pi_{S}=\left(d y, z_{x} d x+z_{y} d y\right)$.

Next observe that, since $\gamma_{B}$ is a Type B path, (6.3) implies that $\mathcal{W}^{1}$ is transverse to $\mathcal{M}^{S}$ at $p$. Thus $\mathcal{W}^{1} \cap \mathcal{M}^{S}$ is a 1-dimensional manifold near $p$. On the other hand, $\pi_{S}: \mathcal{M}^{S} \rightarrow S$ is a map between 2-manifolds, and the above formula shows that $\operatorname{rank} d \pi_{S} \geq 1$. The proof of Proposition 5.3 a then shows that the set of critical points of $\pi_{S}$ is contained in $\mathcal{W}^{1} \cap \mathcal{M}^{S}$.

In coordinates, the intersection $\mathcal{W}^{1} \cap \mathcal{M}^{S}$ is locally the zero locus of the function

$$
w(x, y)=z_{x}(x, y),
$$

while $T_{p}\left(\mathcal{W}^{1} \cap \mathcal{M}^{S}\right)=T_{p} \mathcal{W}^{1} \cap T_{p} \mathcal{M}^{S}$ is the kernel of the 1-form on $S$

$$
d w=z_{x x} d x+z_{y x} d y=2 a d x+b d y
$$

at the origin. Consequently, since $(\kappa, 0)=\partial_{x}$ and $\left(0, \dot{\gamma}_{B}(0)\right)=\partial_{y}$, we have

- $2 a=d w(\kappa, 0) \neq 0$ by (5.15) because $p \notin \mathcal{A}$ so $(\kappa, 0) \notin T_{p} \mathcal{W}^{1}$.

- $b=d w\left(0, K_{B}\right) \neq 0$ by $(6.3 \mathrm{p})$ because $\widetilde{\gamma}_{B}$ is transverse to $\mathcal{W}^{1}$.

- $c=0$ because, for small $t$, the path $\tilde{\gamma}_{B}(t)=(0, t, 0)$ lies in $\mathcal{M}^{S}$, so 6.9 becomes $0=$ $c t^{2}+\mathcal{O}\left(t^{3}\right)$ for all small $t$.

In fact, the expansion in the third bullet point shows that $r_{2}(0, t)=0$ for all small $t$, which implies that 6.9 has the form

$$
z(x, y)=x(a x+b y+r(x, y)),
$$

where $r$ vanishes to first order.

To apply Theorem 6.2 and its corollary, we will need several statements about generic paths in $\mathcal{J}$. These are consequences of the Sard-Smale Theorem ( $\underline{\mathrm{S}}$, [MS, Theorem A.5.1]) applied in the following manner. Suppose that $\pi: \mathcal{M} \rightarrow \mathcal{J}$ and $\rho: \mathcal{N} \rightarrow \mathcal{M}$ are Fredholm maps of separable $C^{\ell}$ Banach manifolds of index $\iota_{\pi}$ and $\iota_{\rho}$ respectively, and with $\ell \geq 2+\max \left(0, \iota_{\pi}, \iota_{\pi}+\iota_{\rho}\right)$. Fix points $J_{0}, J_{1} \in \mathcal{J}$, and let $\mathcal{P}=\mathcal{P} \mathcal{J}$ be the space of $C^{k}, k \gg 1$, paths $[0,1] \rightarrow \mathcal{J}$ from $J_{0}$ to $J_{1}$, which is a separable Banach manifold.

In this context, we have the following lemma.

Lemma 6.4. Assume $J_{0}, J_{1}$ are regular values of $\pi$ and $J_{0}, J_{1} \notin(\pi \circ \rho)(\mathcal{N})$. Then there exists a Baire set $\mathcal{P}^{*}$ of $\mathcal{P}$ so that for each $\gamma \in \mathcal{P}^{*}, \mathcal{M}^{\gamma}=\pi^{-1}(\gamma)$ is an $\iota_{\pi}+1$ dimensional submanifold of $\mathcal{M}$ transverse to $\rho$. In particular, when $\iota_{\rho}+\iota_{\pi} \leq-2, \mathcal{M}^{\gamma}$ is disjoint from $\rho(\mathcal{N})$. Consequently, for each subset $S \subset \mathcal{M}$ of codimension $\geq 2$, there is a Baire set $\mathcal{P}_{S}^{*}$ so that for each $\gamma \in \mathcal{P}_{S}^{*}, \mathcal{M}^{\gamma}$ is a manifold disjoint from $S$.

Proof. The evaluation map ev $: \mathcal{P} \times I \rightarrow \mathcal{J}$ is a submersion of separable Banach manifolds away from the boundary $\mathcal{P} \times \partial I$, while the image $\operatorname{ev}(\mathcal{P} \times \partial I)=\left\{J_{0}, J_{1}\right\}$ of the boundary consists of regular values of $\pi$. It follows that the map

$$
\varphi=\mathrm{ev} \times \pi: \mathcal{P} \times I \times \mathcal{M} \rightarrow \mathcal{J} \times \mathcal{J}
$$


is transverse to the diagonal $\Delta_{\mathcal{J}}$, so the fiber product

$$
\widetilde{\mathcal{M}}=\varphi^{-1}\left(\Delta_{\mathcal{J}}\right)=\left\{(\gamma, t, f) \mid f \in \mathcal{M}^{\gamma(t)}\right\}
$$

is a separable Banach manifold whose boundary is the fiber product of ev $\left.\right|_{\mathcal{P} \times \partial I}$ and $\pi$. Furthermore, the projection $\tilde{\pi}: \widetilde{\mathcal{M}} \rightarrow \mathcal{P}$ is a Fredholm map of index $\iota_{\pi}+1$, while the projection $p_{3}: \widetilde{\mathcal{M}} \rightarrow \mathcal{M}$ onto the third factor is a submersion away from the boundary:

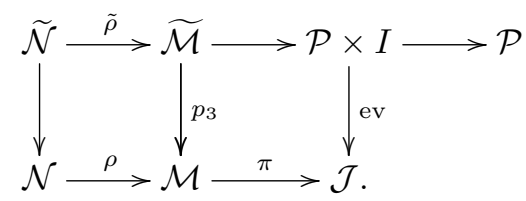

But by assumption, $\pi \circ \rho(\mathcal{N})$ is disjoint from the image $\left(\pi \circ p_{3}\right)(\partial \widetilde{\mathcal{M}})=\left\{J_{0}, J_{1}\right\}$ of the boundary. Therefore the fiber product $\widetilde{\mathcal{N}}$ of $\rho$ and $p_{3}$ is a manifold and the index of $\tilde{\rho}$ is the index of $\rho$.

By Sard-Smale Theorem applied to $\widetilde{\pi}: \widetilde{\mathcal{M}} \rightarrow \mathcal{P}$, there is a Baire subset $\mathcal{P}_{1}$ of $\mathcal{P}$ such that each point $\gamma \in \mathcal{P}_{1}$ is a regular value of $\tilde{\pi}$. It is straightforward to check that $\gamma \in \mathcal{P}$ is a regular value of $\tilde{\pi}$ if and only if the path $\gamma$ is transverse to $\pi: \mathcal{M} \rightarrow \mathcal{J}$. When $\gamma$ is a submanifold of $\mathcal{J}$, this latter transversality means that $\mathcal{M}^{\gamma}=\pi^{-1}(\gamma)$ is a submanifold of $\mathcal{M}$.

Similarly, again by the Sard-Smale Theorem, there is a Baire subset $\mathcal{P}_{2}$ of $\mathcal{P}$ such that each point $\gamma \in \mathcal{P}_{2}$ is a regular point of the composition $\widetilde{\mathcal{N}} \rightarrow \mathcal{P}$ in the top row of 6.10 , and again this occurs if and only if $\gamma$ is transverse to $\pi \circ \rho$. (Note that $J_{0}, J_{1}$ are regular values of $\pi \circ \rho$ because they are not in the image of $\pi \circ \rho$.) When $\gamma$ is a submanifold, this last transversality implies that $\mathcal{N}^{\gamma}=(\pi \circ \rho)^{-1}(\gamma)=\rho^{-1}\left(\mathcal{M}^{\gamma}\right)$ is a submanifold.

Finally, the collection $\mathcal{P}_{3}$ of embedded paths is open and dense in $\mathcal{P}$. Thus $\mathcal{P}^{*}=\mathcal{P}_{1} \cap \mathcal{P}_{2} \cap \mathcal{P}_{3}$ is a Baire set. For each $\gamma \in \mathcal{P}^{*}, \mathcal{M}^{\gamma}$ is an $\iota_{\pi}+1$ dimensional manifold, $\mathcal{N}^{\gamma}$ is an $\iota_{\pi}+1+\iota_{\rho}$ dimensional manifold, and is empty if $\iota_{\pi}+1+\iota_{\rho}<0$. The last statement of the lemma follows from Definition 5.4 and the fact that every countable intersection of Baire sets is a Baire set.

We will apply this reasoning twice: first for paths in $\mathcal{J}=\mathcal{J}^{l}$, then for paths in $\mathcal{J}_{C}$. Recall the notations $\mathcal{J}_{E}^{*}$ and $\mathcal{J}_{\text {isol }}^{E}$ from Section 1. For simplicity, we omit the $X$ from the notation $\mathcal{M}(X)$ of the moduli spaces in Lemmas 6.5 and 6.7

Lemma 6.5. Any path in $\mathcal{J}$ with endpoints in $\mathcal{J}_{E}^{*}$ can be deformed, keeping its endpoints, to a path $\gamma$ such that $\mathcal{M}_{\text {simple }}^{\gamma, E}$ is a 1-dimensional manifold, consisting of embeddings, and intersecting the wall $\mathcal{W}$ transversely in isolated points, all in $\mathcal{W}^{1} \backslash \mathcal{A}$. Moreover, any path $\gamma$ with these properties is in $\mathcal{J}_{\text {isol }}^{E}$.

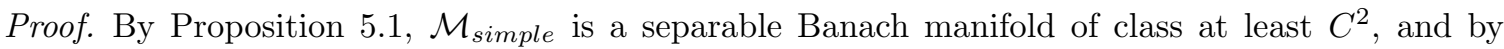
Corollary A.5 the set $\overline{\mathcal{N}}$ of all non-embedded simple maps is a codimension 2 subset. Next, restrict to $\mathcal{M}_{e m b}$, noting that $\mathcal{W}^{1}$ is a submanifold of $\mathcal{M}_{\text {emb }}$ by Proposition $5.3 \mathrm{~b}$, and that $\mathcal{A} \subset$ $\mathcal{M}_{\text {emb }}$ is a codimension 2 submanifold by Lemma 5.6. Together with Lemma 5.5, this shows that $\mathcal{A} \cup \mathcal{N} \mathcal{E} \cup\left(\mathcal{W} \backslash \mathcal{W}^{1}\right)$ is a set of codimension 2 in $\mathcal{M}_{\text {simple }}$.

Now apply Lemma 6.4 with $\mathcal{M}=\mathcal{M}_{\text {simple }}$ and $\mathcal{N}$ equal to the disjoint union $\mathcal{A} \sqcup \mathcal{N} \mathcal{E} \sqcup\left(\mathcal{W} \backslash \mathcal{W}^{1}\right)$, noting that the index $\iota_{\pi}=0$ and $\iota_{\rho} \leq-2$ in this case. This gives a Baire subset $\mathcal{P}_{1}$ of $\mathcal{P}$ over which $\pi^{-1}(\gamma)$ is a manifold of dimension 1 that does not intersect $\mathcal{N}$. Again apply Lemma 6.4 now with $\mathcal{M}=\mathcal{M}_{\text {emb }}$ and $\mathcal{N}$ equal to the codimension 1 submanifold $\mathcal{W}^{1}$. This gives a second Baire subset $\mathcal{P}_{2}$ of $\mathcal{P}$ over which $\pi^{-1}(\gamma)$ is a manifold of dimension 1 that is transverse to $\mathcal{N}$.

Consequently, for each $\gamma$ in the Baire set $\mathcal{P}_{1} \cap \mathcal{P}_{2}, \mathcal{M}_{\text {simple }}^{\gamma, E}$ is a 1-manifold (with boundary) transverse to $\mathcal{W}^{1}$, intersecting the wall $\mathcal{W}$ only along $\mathcal{W}^{1} \backslash \mathcal{A}$, and consisting only of embedded curves. Note that the only critical points of $\pi_{\gamma}: \mathcal{M}_{\text {simple }}^{\gamma, E} \rightarrow[0,1]$ are these wall-crossing points. The proof is completed by observing that the local model $(6.5)$ shows that the wall crossing points 
are non-degenerate critical points of $\pi$ and therefore (a) they are isolated points of $\mathcal{M}_{\text {simple }}^{\gamma, E}$, and (b) all points of the fiber $\mathcal{M}_{\text {simple }}^{\gamma(t), E}$ are isolated for each $t$.

Corollary 6.6. $\mathcal{J}_{\text {isol }}^{E}$ is a dense and path-connected subspace of $\mathcal{J}$.

Proof. Density was shown in Corollary 1.4. Path-connectedness follows from Lemma 6.5 and the fact that $\mathcal{J}$ is path-connected.

We conclude this section by proving a version of Lemma 6.5 for paths in the subspace $\mathcal{J}_{C}$ of $\mathcal{J}$.

Lemma 6.7. Any path in $\mathcal{J}_{C}$ with endpoints in $\mathcal{J}_{E}^{*}$ can be deformed, keeping its endpoints, to a path $\gamma$ in $\mathcal{J}_{C} \cap \mathcal{J}_{\text {isol }}^{E}$ whose lift $\widetilde{\gamma}$ intersects $\mathcal{W}$ transversally at finitely many points, all in $\mathcal{W}^{1} \backslash \mathcal{A}$.

Proof. First consider the subset $\mathcal{M}_{C}=\left\{\iota_{C}\right\} \times \mathcal{J}_{C}$. This is a submanifold of $\mathcal{M}_{\text {emb }}$ that is transverse to $\mathcal{W}^{1}$ by Proposition 5.3c, and to $\mathcal{A}$ by Lemma 5.6. Furthermore, Lemma 5.5 shows that $(\mathcal{W}$ $\left.\mathcal{W}^{1}\right) \cap \mathcal{M}_{C}$ is a codimension 2 subset of $\mathcal{M}_{C}$. By Lemma 6.4 there is a Baire subset of paths $\gamma$ in $\mathcal{J}_{C}$ for which the lift $\widetilde{\gamma}=\left(\iota_{C}, \gamma\right)$ to $\mathcal{M}_{\text {simple }}$ intersects the wall only along $\mathcal{W}^{1} \backslash \mathcal{A}$, and this intersection is a finite set of transverse points. For each intersection point $\widetilde{\gamma}(t) \in \mathcal{W}^{1} \backslash \mathcal{A}$, the local model (6.7) implies that the core curve $\widetilde{\gamma}(t)$ is an isolated point of $\mathcal{M}^{\gamma(t)}$ (and is clearly embedded). The same conclusion is true for those $t$ with $\widetilde{\gamma}(t) \notin \mathcal{W}$ by Proposition 5.3 a.

It remains to find another Baire subset of paths $\gamma$ for which the points of $\mathcal{M}^{\gamma(t)} \backslash \mathcal{M}_{C}$ are embedded and isolated for each $t$. Denote by $\mathcal{M}_{\text {simple }}^{*} \rightarrow \mathcal{J}_{C}$ the moduli space of simple $J$-holomorphic maps $f$ that have at least one point $x_{i}$ on each component of their domain with $f\left(x_{i}\right) \in X \backslash C$. The results of Propositions 5.1 and 5.3, Lemmas 5.5 and 5.6, and Corollary A.5 all extend to the moduli space $\mathcal{M}_{\text {simple }}^{*} \rightarrow \mathcal{J}_{C}$ by using variations supported around the points $f\left(x_{i}\right)$, but vanishing along $C$; such variations are tangent to $\mathcal{J}_{C}$. As in the proof of [MS, Lemma 3.4.3] a further variation, with support off $C$, can be used to ensure that all curves in $\mathcal{M}_{\text {simple }}^{*}$ are transverse to $C$. Again, such variations are tangent to $\mathcal{J}_{C}$.

With this understood, the proof of Lemma 6.5 extends to give a Baire subset $\mathcal{P}^{*}$ of the space $\mathcal{P} \mathcal{J}_{C}$ of paths in $\mathcal{J}_{C}$ so that for each $\gamma \in \mathcal{P}^{*}$, the points of $\mathcal{M}_{\text {simple }}^{\gamma(t), E}$ are embedded and isolated for all $t$.

\section{THE Cluster ISOTOPy THEOREM}

For notational simplicity, given two clusters $\mathcal{O}=(C, \varepsilon, J)$ and $\mathcal{O}^{\prime}=\left(C^{\prime}, \varepsilon^{\prime}, J^{\prime}\right)$ whose core curves $C$ and $C^{\prime}$ have the same genus and homology class, write

$$
G W^{E}(\mathcal{O}) \approx G W^{E}\left(\mathcal{O}^{\prime}\right)
$$

to mean that the difference is a finite sum of terms of the form $\pm G W^{E}\left(C_{i}, \varepsilon_{i}, J_{i}\right)$ of strictly higher level 1.10 compared to that of $C$. With this notation, for example, the conclusion of the cluster refinement Corollary 2.5 simply says that for generic $0<\varepsilon^{\prime}<\varepsilon$

$$
G W^{E}(C, \varepsilon, J) \approx G W^{E}\left(C, \varepsilon^{\prime}, J\right) .
$$

We now use the results of Sections 5 and 6 and an isotopy argument to prove that the GW series of every cluster is equivalent, in the above sense, to the series of an elementary cluster. Recall that, for an elementary cluster $\mathcal{O}_{\text {elem }}, G W\left(\mathcal{O}_{\text {elem }}\right)$ is the universal series

$$
G W_{g}^{\text {elem }}\left(q^{C}, t\right)
$$

given by (3.4) and (3.5) with $q=q^{C}$. In general, we call a cluster $(C, J, \varepsilon)$ regular if the embedding $C \hookrightarrow X$ is a regular $J$-holomorphic map. 
Theorem 7.1 (Cluster Isotopy). For a regular cluster $\mathcal{O}=\left(C, J_{0}, \varepsilon_{0}\right)$ centered at an embedded genus $g J_{0}$-holomorphic curve $C$,

$$
G W^{E}(\mathcal{O}) \approx \operatorname{sign}\left(C, J_{0}\right) G W_{g}^{\text {elem, } E}\left(q^{C}, t\right),
$$

where $G W_{g}^{\text {elem, }}$ is the truncation of 7.2 below energy $E$.

Proof. The proof of Proposition 3.2 shows that there exist $J_{1} \in \mathcal{J}_{C}$ and $\varepsilon_{1}$ so that $\mathcal{O}_{\text {elem }}=\left(C, \varepsilon_{1}, J_{1}\right)$ is an elementary cluster. In fact, we can assume that $J_{1} \in \mathcal{J}_{C} \cap \mathcal{J}_{E}^{*}$ after a perturbation supported outside the $\varepsilon_{1} / 2$-neighborhood of $C$ of the type constructed in the proof of [MS, Lemma 3.4.3]. Choose a path $\gamma(t)=J_{t}$ in $\mathcal{J}_{C}$ from $J_{0}$ to $J_{1}$ (the proof of Theorem A.2 of [IP2] shows that $\mathcal{J}_{C}$ is connected). By Lemma 6.7 we can assume, after a deformation, that $\gamma$ is a path in $\mathcal{J}_{C} \cap \mathcal{J}_{\text {isol }}^{E}$ and there is a finite set Sing $=\left\{t_{i}\right\}$, not containing 0 or 1 , such that $\tilde{\gamma}=\left(f_{C}, \gamma(t)\right)$

- lies in $\mathcal{M}^{\gamma} \backslash \mathcal{W}$ for all $t \notin$ Sing, and

- lies in a 2-dimensional surface $V_{i}$ given by (6.6) for $t \in\left[t_{i}-\delta, t_{i}+\delta\right]$.

Choose $\delta>0$ small enough so that the intervals $\left[t_{i}-\delta, t_{i}+\delta\right]$ do not overlap, and let their endpoints be $0<\tau_{1}<\cdots<\tau_{2 k}<1$. For each $i$, fix a cluster $\mathcal{O}_{i}=\left(C, \varepsilon_{i}, J_{\tau_{i}}\right)$. Then $\widetilde{\gamma}$ can be regarded as the composition of paths $\widetilde{\gamma}_{i}:\left[\tau_{i}, \tau_{i+1}\right] \rightarrow \mathcal{M}^{\gamma}$ of two types:

(i) Paths in $\mathcal{M}_{e m b} \backslash \mathcal{W}$. For these, Lemma 7.2 below shows that $G W^{E}\left(\mathcal{O}_{i}\right) \approx G W^{E}\left(\mathcal{O}_{i+1}\right)$.

(ii) Paths in $\mathcal{M}_{e m b} \cap V_{i}$, crossing the wall transversally at a single point of $\mathcal{W}^{1} \backslash \mathcal{A}$. For these, Lemma 7.4 below shows that $G W^{E}\left(\mathcal{O}_{i}\right) \approx-G W^{E}\left(\mathcal{O}_{i+1}\right)$.

Altogether, we conclude that

$$
G W^{E}(\mathcal{O}) \approx(-1)^{\sigma} G W^{E}\left(\mathcal{O}_{\text {elem }}\right),
$$

where $\sigma$ is the number of transverse wall-crossings, which is exactly the spectral flow of the operator $D_{p}$ along the path $\tilde{\gamma}$. The path ends at an elementary cluster, which has positive sign by (3.6). Thus $(-1)^{\sigma}$ is exactly the sign of the initial curve $\left(C, J_{0}\right)$.

In the above proof, the assertion in Step (i) is a fact about isotopies with no wall-crossings. It can be stated as follows.

Lemma 7.2 (Simple Isotopy). Fix $E>0$. Then for any path $\left(C_{t}, J_{t}\right)$ in $\mathcal{M}_{\text {emb }} \backslash \mathcal{W}$ with $J_{t}$ in $\mathcal{J}_{\text {isol }}^{E}$ and any $\varepsilon_{0}, \varepsilon_{1}$ such that $\left(C_{0}, \varepsilon_{0}, J_{0}\right)$ and $\left(C_{1}, \varepsilon_{1}, J_{1}\right)$ are clusters,

$$
G W^{E}\left(C_{0}, \varepsilon_{0}, J_{0}\right) \approx G W^{E}\left(C_{1}, \varepsilon_{1}, J_{1}\right) \text {. }
$$

Proof. It follows from Proposition 5.3 and the compactness of $[0,1]$ that there is a $\delta>0$ such that, for each $t \in[0,1], C_{t}$ is the only $J_{t}$-holomorphic curve in its degree and genus in the ball $B\left(C_{t}, \delta\right)$ (in Hausdorff distance). By Lemma 2.3 we can choose, for each $0 \leq t \leq 1$, an $0<\varepsilon_{t}<\delta$ such that $\left(C_{t}, \varepsilon_{t}, J_{t}\right)$ is a cluster. Then, by Lemma 2.1. $\left(C_{s}, \varepsilon_{t}, J_{s}\right)$ has a well-defined contribution $G W^{E}\left(C_{s}, \varepsilon_{t}, J_{s}\right)$ for all $s$ in an open interval around $t$. These open intervals cover [0,1]; take a finite subcover $\left\{I_{k}\right\}$. Then $G W^{E}\left(C_{s}, \varepsilon_{k}, J_{s}\right)$ is constant for $s$ in each $I_{k}$ and $C_{s}$ is the only $J_{s}$-holomorphic curve in its genus and homology class in that ball. Corollary 2.5 shows that on the intersection of two consecutive intervals the corresponding $G W^{E}$ invariants differ by the contributions of higher-level clusters. The lemma follows.

By Lemma 6.5, each path in $\mathcal{J}$ with endpoints in $\mathcal{J}_{E}^{*}$ can be deformed, keeping its endpoints, to a path $\gamma$ in $\mathcal{J}_{\text {isol }}^{E}$ such that the projection

$$
\pi_{\gamma}: \mathcal{M}_{e m b}^{\gamma} \rightarrow \gamma
$$

has only non-degenerate critical points, none an endpoint, each locally modeled by (6.5). If $a>0$ in the local model, then $\gamma$ can be parameterized so that $\gamma(t)=t$ and $\pi^{-1}(t)=\left\{x \mid t=a x^{2}\right\}$ is empty for $t<0$ and is two distinct curves $C_{t}^{ \pm}$for $0<t<\delta$ (and vice versa if $a<0$ ). A second isotopy lemma relates the GW invariants of clusters centered on these curves $C_{t}^{ \pm}$. 
Lemma 7.3 (Wall-crossing in $\mathcal{J}$ ). Fix $E>0$, a path $\gamma$ in $\mathcal{J}_{\text {isol }}^{E}$ and a non-degenerate critical point $\left(C_{0}, J_{0}\right)$ of $(7.5)$ for $J_{0}=\gamma(0)$. Then there exists a $\delta>0$ and a neighborhood $U$ of $\left(C_{0}, J_{0}\right)$ in $\mathcal{M}$ such that if $0 \neq|t|<\delta$ and the sign of $t$ is such that $\mathcal{M}^{\gamma(t)} \cap U=\left\{C_{t}^{ \pm}\right\}$, then the two clusters $\mathcal{O}^{+}=\left(C_{t}^{+}, \varepsilon, J_{t}\right), \mathcal{O}^{-}=\left(C_{t}^{-}, \varepsilon^{\prime}, J_{t}\right)$ satisfy

$$
G W^{E}\left(\mathcal{O}^{+}\right) \approx-G W^{E}\left(\mathcal{O}^{-}\right) .
$$

Proof. The local model 6.5 at $\left(C_{0}, J_{0}\right)$ implies that there is an $\varepsilon_{1}>0$ and a ball $U=B\left(C_{0}, \varepsilon_{1}\right)$ in $\mathcal{C}(X)$ that contains $C_{t}^{ \pm}$and no other $J_{t}$ holomorphic curves in the degree and genus of $C_{0}$ for all $|t|<\varepsilon_{1}$. Because $J_{t} \in \mathcal{J}_{\text {isol }}^{E}$, Lemma 2.3 ensures that $\varepsilon_{1}$ can be chosen so that $\left(C_{0}, \varepsilon_{1}, J_{0}\right)$ is a cluster. As $J$ varies, the associated invariant $G W^{E}\left(U, J_{s}\right)$ is, by Lemma 2.1. well-defined and independent of $s$ for small $s$.

The local model (6.5) shows that $U \cap \mathcal{C}^{J_{s}, E}$ is $\left\{C_{t}^{ \pm}\right\}$for $s=t$, and is empty for $s=-t$. Taking $s=t$ and applying Proposition 2.4 one sees that $U$ decomposes into two clusters $\mathcal{O}_{t}^{ \pm}$with $G W^{E}\left(\mathcal{O}^{+}\right)+G W^{E}\left(\mathcal{O}^{-}\right) \approx G W^{E}\left(U, J_{s}\right)$. Applying the same theorem with $s=-t$ shows that $G W^{E}\left(U, J_{s}\right) \approx 0$. The proof is completed by noting that

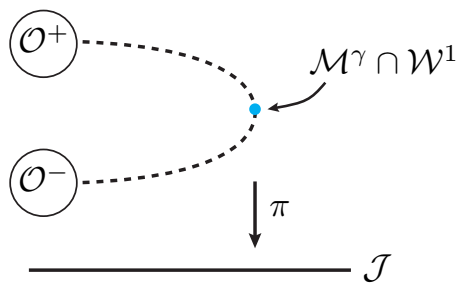
the invariants $G W^{E}\left(\mathcal{O}^{ \pm}\right)$satisfy 7.1 as $\varepsilon$ and $\varepsilon^{\prime}$ vary.

The core curve of a cluster does not persist through the wall-crossing described by Lemma 7.3 . But the core curve remains if we fix the complex structure on the core curve $C$ and cross the wall along a path $\gamma$ in $\mathcal{J}_{C}$, as was done in the proof of Theorem 7.1. After a perturbation as in Lemma6.7. the wall-crossing is locally modeled by (6.7). In this picture, for each $0<t<\delta$, there are four curves to consider: the incoming core curve $\left(C, J_{-t}\right)$, the outgoing core curve $\left(C, J_{t}\right)$, and a second pair of curves $\left(C_{-t}^{\prime}, J_{-t}\right)$ and $\left(C_{t}^{\prime}, J_{t}\right)$.

Lemma 7.4 (Wall-crossing in $\mathcal{J}_{C}$ ). Fix $E>0$ and a path $\gamma$ in $\mathcal{J}_{C} \cap \mathcal{J}_{\text {isol }}^{E}$ so that $\widetilde{\gamma}(t)$ crosses the wall transversally at $t=0$ at a point $\left(C, J_{0}\right)$ in $\mathcal{W}^{1} \backslash \mathcal{A}$. Then there exists a $\delta>0$ so that each incoming cluster $\mathcal{O}_{-\delta}=\left(C, \varepsilon, J_{-\delta}\right)$ and each outgoing cluster $\mathcal{O}_{\delta}=\left(C, \varepsilon^{\prime}, J_{\delta}\right)$ satisfy

$$
G W^{E}\left(\mathcal{O}_{-\delta}\right) \approx-G W^{E}\left(\mathcal{O}_{\delta}\right) .
$$

Proof. Consider the local model $\mathcal{M}^{S} \rightarrow S$ given by (6.6). Its restriction over $\gamma$, given by (6.7), is two curves crossing at the origin. We will perturb this level set $\{z=0\}$ in two opposite directions.
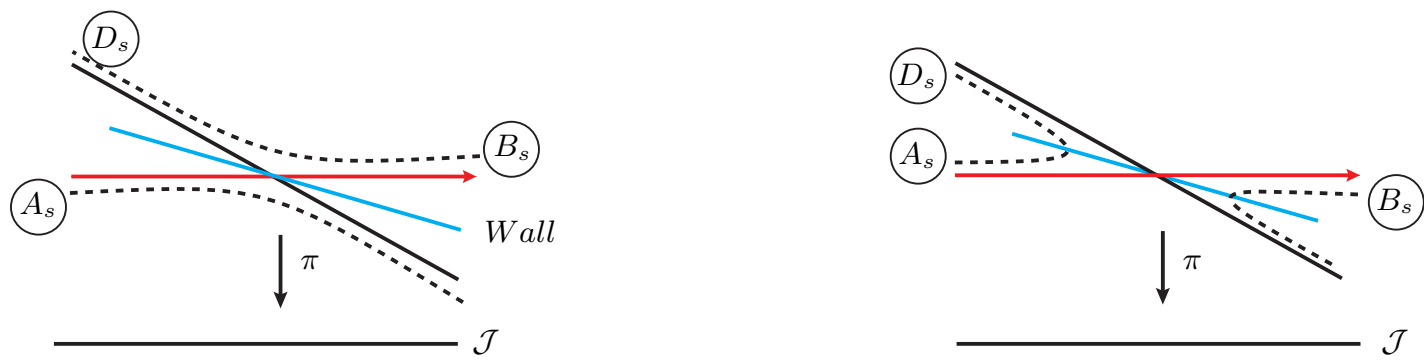

Figure 4 . These figures show the curve $C$ (horizontal line) and curves $C_{t}^{\prime}$ (diagonal line) as $t$ (the horizontal coordinate) varies. The circled labels refer to clusters at the ends of the dotted paths, with $s>0$ in the first figure, $s<0$ in the second, and $a, b>0$ in both.

In the chart (6.6), with $\delta>0$ fixed and small, $\left(C, J_{-\delta}\right)$ has coordinates $(0,-\delta, 0)$, so can be perturbed to $\left(C_{-\delta, s}, J_{-\delta, s}\right)$ with coordinates $(x(s),-\delta, s)$, where $x \approx-s / b \delta$ is the unique solution 
of $s=x(a x-b \delta+r(x,-\delta))$ with $x=O(s)$. The curves $\left(C_{-\delta}^{\prime}, J_{-\delta}\right)$ and $\left(C, J_{\delta}\right)$ can be similarly perturbed. By Lemma 2.3, the GW invariants of the corresponding clusters

$$
A_{s}=\left(C_{-\delta, s}, \varepsilon, J_{-\delta, s}\right), \quad B_{s}=\left(C_{\delta, s}, \varepsilon, J_{\delta, s}\right), \quad D_{s}=\left(C_{-\delta, s}^{\prime}, \varepsilon, J_{\delta, s}\right)
$$

are locally constant in $s$ : for sufficiently small $s$ (and $\delta>0$ ) we have

$$
G W^{E}\left(A_{s}\right)=G W^{E}\left(\mathcal{O}_{-\delta}\right), \quad G W^{E}\left(B_{s}\right)=G W^{E}\left(\mathcal{O}_{\delta}\right), \quad G W^{E}\left(D_{s}\right)=G W^{E}\left(D_{-s}\right) .
$$

Assume $a>0$ (else change $s \rightarrow-s$ ), and that $b>0$ (else change $t \rightarrow-t$ ). The moduli space $\mathcal{M}^{S}$ over $S$ is locally near $\left(C, J_{0}\right)$ the level set

$$
\{(x, t, s) \mid s=x(a x+b t+r(x, t))\} .
$$

For each fixed $s, t$ small, this quadratic equation in $x$ has either no solution or two solutions, except at a single point $x \approx-b t / 2 a$, where the tangent is in the kernel of the projection to $\mathcal{J}$, which means that this point lies on the wall and is a non-degenerate critical point of 7.5 .

For a small positive $s$, the moduli space over $\gamma_{s}(t)=(t, s),-\delta \leq t \leq \delta$ therefore contains a path in $\mathcal{M}_{e m b}$ from the core of cluster $D_{s}$ to the core of $B_{s}$ that does not cross the wall. After a small perturbation using Lemma 6.5. Lemma 7.2 applies to give:

$$
G W^{E}\left(D_{s}\right) \approx G W^{E}\left(B_{s}\right) .
$$

For a small negative $s$ the moduli space over $\gamma_{s}(t)=(t, s),-\delta \leq t \leq 0$ is a path in $\mathcal{M}_{\text {emb }}$ from the core of cluster $A_{s}$ to the core of $D_{s}$, crossing now the wall transversally (at a point in $\mathcal{W}^{1} \backslash \mathcal{A}$ ). Perturbing $\gamma_{s}$ by Lemma 6.5 gives a path in $\mathcal{J}_{\text {isol }}$ so Lemma 7.3 applies in this case to give

$$
G W^{E}\left(A_{s}\right) \approx-G W^{E}\left(D_{s}\right) .
$$

The proof is completed by combining (7.6, (7.7) and 7.8.

\section{Structure theorems And the proof of the GV Conjecture}

The isotopy results of the previous section lead quickly to a formula 8.1 that shows that the GW invariants have a remarkably simple structure. This formula is compatible with a simple geometric picture: if one could find a $J \in \mathcal{J}$ so that all simple $J$-holomorphic maps in $X$ were elementary, then $G W(X)$ would have exactly the form $(8.1)$, with $e_{A, g}(X)$ equal to the count of $J$-holomorphic curves with homology class $A$ and genus $g$. However, it is far from clear whether any such $J$ exists. Thus the coefficients $e_{A, g}(X)$ can be regarded as virtual counts of elementary clusters in $X$.

Theorem 8.1. For any closed symplectic Calabi-Yau 6-manifold $X$, there exist unique integer invariants $e_{A, g}(X)$ such that

$$
G W(X)=\sum_{A \neq 0} \sum_{g \geq 0} e_{A, g}(X) \cdot G W_{g}^{\text {elem }}\left(q^{A}, t\right) .
$$

Proof. The uniqueness of the coefficients in (8.1) is easily shown because the collection of series

$$
G W_{g}^{\text {elem }}\left(q^{A}, t\right)=t^{2 g-2} q^{A}\left(1+\text { higher order in } t \text { and } q^{A}\right)
$$

for $g \geq 0$ and $A \in H_{2}(X, \mathbb{Z})$ is linearly independent. To prove existence, fix $E$, choose any parameter $J \in \mathcal{J}_{E}^{*}$, and use Proposition 2.4 to write $G W^{E}(X)$ as a sum of finitely many cluster contributions. Formula (8.1) follows from the corresponding formula for each cluster, which is proved in Lemma 8.2 below, by taking $E \rightarrow \infty$. 
Lemma 8.2. For any regular $E$-cluster $\mathcal{O}$ centered at a genus $g$ curve $C$ there exist unique integers $e_{d, h}(\mathcal{O})$, beginning with $e_{1, g}(\mathcal{O})=\operatorname{sign}(C)$, such that

$$
G W^{E}(\mathcal{O})=\sum_{d \geq 1} \sum_{h \geq g} e_{d, h}(\mathcal{O}) G W_{h}^{\text {elem }, E}\left(q^{d C}, t\right),
$$

where both sides are truncated below energy level $E$.

Proof. Because all $J$-holomorphic maps in $\mathcal{O}$ represent $k[C]$ and have genus at least $g, G W^{E}(\mathcal{O})$ has the form

$$
G W^{E}(\mathcal{O})=\sum_{k \geq 1} \sum_{h \geq g} G W_{k, h}^{E}(\mathcal{O}) q^{k C} t^{2 h-2}
$$

with $k \omega(C) \leq E$ and $h \leq E$. Define the $(C, g)$-relative level of the monomial $t^{2 h-2} q^{k C}$ to be $\Omega(k)+h-g$, and note that all terms in (8.4) have non-negative relative level.

Using this series (8.4), we define the truncation $\left[G W^{E}(\mathcal{O})\right]_{m}$ of the lefthand side of $(8.3)$ to be the sum of the terms in (8.4) with $(C, g)$-relative level $\Omega(k)+h-g \leq m$. The righthand side of 8.3 . can be similarly truncated. In fact, by $(8.2)$ the truncation of $(8.3)$ involves only those $e_{d, h}(\mathcal{O})$ with $\Omega(d)+h-g \leq m$. We will prove the lemma using complete induction on $m$.

The induction begins with $m=-1$; in this case, the truncations of both sides of 8.3 vanish. For the induction step, we assume that for every regular cluster $\mathcal{O}$, whose core curve corresponds to any $(A, g)$, there are coefficients $e_{d, h}(\mathcal{O}) \in \mathbb{Z}$ such that 8.3 holds when truncated at $(A, g)$-relative level $m-1$. Now by Theorem 7.1 we have

$$
G W^{E}(\mathcal{O})= \pm G W_{g}^{\text {elem, } E}\left(q^{C}, t\right)+\sum_{i \in I} \pm G W^{E}\left(\mathcal{O}_{i}\right)
$$

where the $\mathcal{O}_{i}$ are clusters, indexed by a finite set $I$, whose core curves $C_{i}$ have $\left[C_{i}\right]=k_{i}[C]$, genus $g_{i} \geq g$ and $(C, g)$-relative level $m_{i}=\Omega\left(k_{i}\right)+g_{i}-g>0$. When $G W^{E}(\mathcal{O})$ is truncated at relative level $m$, each $G W^{E}\left(\mathcal{O}_{i}\right)$ is truncated at $\left(C_{i}, g_{i}\right)$-relative level $m-m_{i}<m$ so by induction,

$$
G W^{E}(\mathcal{O})= \pm G W_{g}^{\text {elem }, E}\left(q^{C}, t\right)+\sum_{i \in I} \pm\left(\sum_{d, h} e_{d, h}\left(\mathcal{O}_{i}\right) G W_{h}^{\text {elem, } E}\left(q^{d k_{i} C}, t\right)\right)
$$

holds when truncated at $(C, g)$-relative level $m$. This completes the induction step.

In fact, we get the following result for any closed symplectic 6-dimensional manifold $X$ as long as we restrict to the $\mathrm{GW}$ invariants coming only from classes $A \in H_{2}(X, \mathbb{Z})$ with vanishing Chern number $c_{1}(A)=c_{1}(X) A$.

Theorem 8.3. Assume $X$ is a closed symplectic 6-manifold. Then there exist unique integer invariants $e_{A, g}(X)$, defined for homology classes $A$ with $c_{1}(A)=0$, such that the $G W$ invariant of $X$ satisfies

$$
\sum_{\substack{A \neq 0 \\ c_{1}(A)=0}} \sum_{g \geq 0} G W_{A, g}(X) t^{2 g-2} q^{A}=\sum_{\substack{A \neq 0 \\ c_{1}(A)=0}} \sum_{g \geq 0} e_{A, g}(X) \cdot G W_{g}^{\text {elem }}\left(q^{A}, t\right) .
$$

Proof. The dimension 1.3$)$ is $2 c_{1}(A)$, independent of the genus. It suffices to check that all the results in Sections 1-6 continue to hold as long as we replace everywhere $\overline{\mathcal{M}}(X)$ by the union of its zero dimensional pieces

$$
\bigsqcup_{\substack{A \neq 0 \\ c_{1}(A)=0}} \overline{\mathcal{M}}_{A, g}(X)
$$

A dimension count shows that for generic $J$ the limit points of 8.6 in the rough topology (after restricting below fixed energy level $E$ ) can only be multiple covers of points of (8.6), and not of points with $c_{1}(A) \neq 0$. The rest is straightforward, and the details are left to the reader. 
8.1. Proof of the GV conjecture. The GV conjecture follows easily from Theorem 8.1 and the explicit form of the GW invariant of an elementary cluster. For simplicity, set

$$
\mathcal{E}_{h}(q, t)=\sum_{k=1}^{\infty} \frac{1}{k}\left(2 \sin \frac{k t}{2}\right)^{2 h-2} q^{k} .
$$

With this notation, the GW invariant (3.7) of an elementary cluster whose core curve has genus $g$ is

$$
G W_{g}^{\text {elem }}(q, t)=\sum_{d \neq 0} \sum_{h \geq g} n_{d, h}(g) \mathcal{E}_{h}\left(q^{d}, t\right),
$$

where the $n_{d, h}(g)$ are integers by Proposition 3.4 that vanish unless $h \geq g$ by Proposition 3.4b. The GV Conjecture then takes the following form.

Theorem 8.4. Let $X$ be a closed symplectic Calabi-Yau 6-manifold. Then there are unique integers $e_{A, h}(X)$ such that

$$
G W(X)=\sum_{A \neq 0} \sum_{h} e_{A, h}(X) \mathcal{E}_{h}\left(q^{A}, t\right) .
$$

In fact, these BPS numbers $n_{A, h}(X)$ are obtained from the virtual counts $e_{A, h}(X)$ of Theorem 8.1 by the universal formula involving the coefficients $n_{d, h}(g)$ in 8.7$)$ :

$$
n_{A, h}(X)=\sum_{\substack{d, B \\ d B=A}} \sum_{g=0}^{h} e_{B, g}(X) \cdot n_{d, h}(g) \in \mathbb{Z},
$$

where the first sum is over all integers $d \geq 1$ and $B \in H_{2}(X, \mathbb{Z})$ such that $d B=A$ in $H_{2}(X, \mathbb{Z})$.

Proof. This follows immediately by combining (8.1) and (8.7) and rearranging the sums:

$$
\begin{aligned}
G W(X) & =\sum_{A \neq 0} \sum_{g \geq 0} e_{A, g}(X) \sum_{d \geq 1} \sum_{h \geq g} n_{d, h}(g) \mathcal{E}_{h}\left(q^{d A}, t\right) \\
& =\sum_{A \neq 0} \sum_{d \geq 1} \sum_{h}\left(\sum_{g \leq h} e_{A, g}(X) n_{d, h}(g)\right) \mathcal{E}_{h}\left(q^{d A}, t\right) \\
& =\sum_{A \neq 0} \sum_{\substack{d, B \\
d B=A}} \sum_{h}\left(\sum_{g \leq h} e_{B, g}(X) n_{d, h}(g)\right) \mathcal{E}_{h}\left(q^{A}, t\right) .
\end{aligned}
$$

The rearrangements are justified by first working below an energy level $E(A, g) \leq E$, where all sums are finite.

\section{Extensions of The GV Structure theOREM}

This section extends Theorem 8.4 in two different directions: to general symplectic 6 -manifolds, and to the genus zero GW invariants of closed symplectic $n$-manifolds, $n \geq 6$, that are semipositive (as defined in [MS]), a class that includes symplectic Calabi-Yau manifolds. In fact, all transversality results were proved for simple maps in index zero moduli spaces. A version of the Cluster Decomposition Proposition 2.4 holds provided the underlying curve map 1.9 does not increase the dimension of such moduli spaces in the sense described below.

We restrict to the primary $G W$ invariants of $X$, which are defined using the evaluation map (but not the stabilization map) in (1.1). For each collection $\left\{\gamma_{i}\right\} \subset H^{*}(X, \mathbb{Z})$ consider the generating function

$$
G W^{X}\left(\gamma_{1}, \ldots, \gamma_{k}\right)=\sum_{A \neq 0} \sum_{g \geq 0}\left\langle\left[\overline{\mathcal{M}}_{A, g, k}^{J}(X)\right]^{v i r}, \mathrm{ev}^{*}\left(\gamma_{1} \times \ldots \times \gamma_{k}\right)\right\rangle q^{A} t^{2 g-2} .
$$


The pairing is defined to be zero unless the formal dimension is zero, that is, unless $\iota=0$, where

$$
\iota=2 c_{1}(A)+(\operatorname{dim} X-6)(1-g)+2 k-\sum_{i=1}^{k} \operatorname{dim} \gamma_{i} .
$$

As usual, the pairing vanishes unless $\operatorname{dim} \gamma_{i} \geq 2$ for each $i$, so we henceforth assume this inequality. Throughout this section, we assume that $\operatorname{dim} X \geq 6$.

The coefficients in 9.1 are obtained by fixing pseudo-cycles $\beta_{i}: B_{i} \rightarrow X$ representing the Poincaré duals of $\gamma_{i}$ (cf. [MS, Section 6.5]), and restricting to the index $\iota=0$ constrained moduli space

$$
\overline{\mathcal{M}}_{A, g, B}^{J}(X)=\overline{\mathcal{M}}_{A, g, k}^{J}(X) \times_{X^{k}}\left(B_{1} \times \ldots \times B_{k}\right)
$$

(the fiber product of the evaluation map ev $: \overline{\mathcal{M}}_{A, g, k}^{J}(X) \rightarrow X^{k}$ and the map $B_{1} \times \ldots \times B_{k} \rightarrow X^{k}$ ). This gives rise in the usual way to the primary GW invariant that appears as the coefficients in (9.1) (cf. page 197 of [MS]).

Lemma 1.2 remains true for these index 0 constrained moduli spaces in the following form. Let $\mathcal{D}$ be the (countable) set consisting of the indexing data $(A, g, \gamma)$ appearing in (9.1). For each $\gamma=\left(\gamma_{1}, \ldots, \gamma_{k}\right)$, choose a set of pseudo-cycle representatives $\beta_{1}, \ldots, \beta_{k}$ that are in general position. Standard transversality results show that, for each element of $\mathcal{D}$, each open stratum of the constrained universal moduli space

$$
\overline{\mathcal{M}}_{A, g, B}(X)_{\text {simple }} \rightarrow \mathcal{J}
$$

is a manifold. The Sard-Smale Theorem gives a Baire set of regular points in $\mathcal{J}$ for the map (9.4) for each $\mathcal{D}$; after intersecting over the elements of $\mathcal{D}$ we can assume these are regular for all $\mathcal{D}$. Parts (a) and (b) of Lemma A.1, together with the Sard-Smale Theorem, give two similar Baire sets. Another intersection produces a single Baire set $\mathcal{J}^{*}$ of $\mathcal{J}$ such that for each $J \in \mathcal{J}^{*}$ all index 0 moduli spaces 9.3) satisfy:

(a) All simple $J$-holomorphic maps are regular, and are embeddings with pairwise disjoint images that are $B$-regular, meaning that for each $i=1, \ldots, k, f\left(x_{i}\right)$ is a regular value of $\beta_{i}$ and $\left(\beta_{i}\right)_{*}\left(T_{b_{i}} B_{i}\right) \cap f_{*}\left(T_{x_{i}} C\right)=0$ for each $b_{i} \in B_{i}$ with $\beta_{i}\left(b_{i}\right)=f\left(x_{i}\right)$.

(b) The projection (9.4) is a local diffeomorphism around each map that is regular, $B$-regular, and an embedding.

Moreover, for each $J \in \mathcal{J}^{*}$, there are no simple $J$-holomorphic maps in the spaces 9.3 with $\iota<0$. The universal moduli space constrained by $B$ is

$$
\overline{\mathcal{M}}_{B}(X)=\bigsqcup_{A, g} \overline{\mathcal{M}}_{A, g, B}(X)
$$

where the disjoint union is only over those $(A, g)$ for which $\iota$ in 9.2$)$ is zero.

As in the proof of Lemma 1.5, any nontrivial $J$-holomorphic map $f: C \rightarrow X$ has an associated "reduced map" $\varphi: C_{\text {red }} \rightarrow X$, which is a simple $J$-holomorphic map with the same image as $f$. In this context, the underlying curve map (1.8) extends to a map

$$
c: \overline{\mathcal{M}}_{B}(X) \longrightarrow \operatorname{Subsets}(X) \times \mathcal{J} \times B_{1} \times \ldots \times B_{k}
$$

defined by $\left(f, x_{1}, \ldots, x_{k}, J, b_{1}, \ldots, b_{k}\right) \mapsto\left(f(C), J, b_{1}, \ldots, b_{k}\right)$. The examples below give structure theorems in cases where $c$ does not increase the formal dimension $(9.2)$, that is, where $\iota(\varphi) \leq \iota(f)$.

Under this assumption, we can replace $\overline{\mathcal{M}}(X)$ everywhere by 9.5$)$ and all proofs in Sections 1-7, except those in Section 3, hold without change. In particular, there is a dense, path-connected set $\mathcal{J}_{\text {isol }}^{E}(B)$ corresponding to $\mathcal{J}_{\text {isol }}^{E}$ in Definition 1.3 but involving only maps in $\overline{\mathcal{M}}_{B}(X)$. Lemma 1.6 holds under the assumption above with $\mathcal{C}(X)$ replaced by the image of 9.6 .

To finish, we must expand the definitions of "cluster" and "elementary cluster". Define a $B$ constrained cluster exactly as in Definition 2.2 but using only elements $(f, \mathbf{x}, J, \mathbf{b})$ in $\overline{\mathcal{M}}_{B}(X)$. Thus 
the core $C$ is a smooth embedded $J$-holomorphic curve $\iota_{C}: C \rightarrow X$ that we now assume is marked, $B$-regular as defined in (a) above, and decorated by a choice of $b_{i} \in \beta_{i}^{-1}\left(\iota_{C}\left(x_{i}\right)\right)$ for each $i$. The contribution of a $B$-constrained cluster $(C, J, \varepsilon)$ to $G W(\gamma)$ depends only on the restriction of $J$ to the $\varepsilon$ neighborhood of the core curve and the restriction of each $\beta_{i}$ to the $\varepsilon$-ball in $B_{i}$ centered at $b_{i}$, for $i=1, \ldots, k$. By a diffeomorphism, when $\varepsilon$ is small, we can identify the $\varepsilon$ tubular neighborhood of $C$ with an $\varepsilon$-disk bundle of the normal bundle $N_{C} \rightarrow C$, with $C$ mapping to the zero section and each $\varepsilon$-ball around $b_{i}$ in $B_{i}$ mapping into a linear subspace of the fiber $N_{i}$ over the points $p_{i}=\iota_{C}\left(x_{i}\right)$. One can then declare certain $B$-constrained clusters to be "elementary". In both of the examples below there is a simple, natural way of doing this.

9.1. GV-formula for general symplectic 6-manifolds. For any closed symplectic 6-manifold $X$, the dimension 9.2 is

$$
\iota=2 c_{1}(A)+\sum_{i=1}^{k}\left(2-\operatorname{dim} \gamma_{i}\right),
$$

independent of the genus. For the "Calabi-Yau classes" $A$ with $c_{1}(A)=0$ consider the GV-transform

$$
\sum_{\substack{A \neq 0, g \\ c_{1}(A)=0}} G W_{A, g} q^{A} t^{2 g-2}=\sum_{\substack{A \neq 0, g \\ c_{1}(A)=0}} n_{A, g} \sum_{k=1}^{\infty} \frac{1}{k}\left(2 \sin \frac{k t}{2}\right)^{2 g-2} q^{k A} .
$$

For "Fano classes" $A$ with $c_{1}(A)>0$ consider the following variation of the GV transform:

$$
\sum_{\substack{A, g \\ c_{1}(A)>0}} G W_{A, g}\left(\gamma_{1}, \cdots, \gamma_{k}\right) q^{A} t^{2 g-2}=\sum_{\substack{A, g \\ c_{1}(A)>0}} n_{A, g}\left(\gamma_{1}, \cdots, \gamma_{k}\right)\left(2 \sin \frac{t}{2}\right)^{c_{1}(A)+2 g-2} t^{-c_{1}(A)} q^{A}
$$

for each collection $\left\{\gamma_{i}\right\} \subset H^{*}(X, \mathbb{Z})$. The invariants $G W_{A, g}$ are zero for all classes $A$ with $c_{1}(A)<0$ (the moduli space without constraints is empty for $J \in \mathcal{J}^{*}$ ).

Theorem 9.1. For a closed symplectic 6-dimensional manifold $X$, the coefficients of the primary $G W$ series (9.7) and (9.8) have the following integrality properties:

$$
n_{A, g} \in \mathbb{Z} \quad \text { if } c_{1}(A)=0, \quad \text { and } \quad n_{A, g}\left(\gamma_{1}, \cdots, \gamma_{k}\right) \in \mathbb{Z} \quad \text { if } c_{1}(A)>0
$$

for all $\gamma_{1}, \ldots, \gamma_{k} \in H^{*}(X, \mathbb{Z})$.

Proof. Fix $\gamma=\left\{\gamma_{i}\right\}$, corresponding constraints $B=\left\{B_{i}\right\}$, and a class $A \neq 0$ so that

$$
\iota=2 c_{1}(A)+\sum\left(2-\operatorname{dim} \gamma_{i}\right)
$$

is zero. For each $J \in \mathcal{J}^{*}$, the resolution of each $J$-holomorphic map $f$, factors as $\varphi \circ \rho$ as described above, where $\varphi: C_{\text {red }} \rightarrow X$ is a simple $J$-holomorphic map. For each component $\Sigma_{i}$ of $C_{\text {red }}$, let $A_{i}=\varphi_{*}\left[\Sigma_{i}\right] \in H_{2}(X, \mathbb{Z})$, and let $d_{i} \geq 1$ denote the degree of $\rho$ over $\Sigma_{i}$, that is, the number of points in $\rho^{-1}(x)$ for a generic point $x \in \Sigma_{i}$. Then $A=\sum d_{i} A_{i}$.

Because $\varphi: C_{\text {red }} \rightarrow X$ is a simple $J$-holomorphic map, it cannot have any components with $c_{1}\left(A_{i}\right)<0$ (such moduli spaces are empty for $J \in \mathcal{J}^{*}$ ). Moreover, the image of $\varphi$ passes through all the constraints and represents $\sum A_{i}$. Hence

$$
c_{1}\left(\varphi_{*}\left[C_{r e d}\right]\right)=\sum c_{1}\left(A_{i}\right) \leq \sum d_{i} c_{1}\left(A_{i}\right)=c_{1}(A)
$$

in fact this must be an equality because otherwise the formal dimension of the constrained moduli space containing $\varphi$ would be negative, contradiction. Thus for $J \in \mathcal{J}^{*}$, a multiple cover map represents a Calabi-Yau class if and only if its reduced curve also does, and represents a Fano class if and only if its reduction also $\operatorname{does}$ and $\operatorname{deg} \rho=1$.

Consequently, the GW series separates into two independent contributions: a sum over the CalabiYau classes, where Theorem 8.3 applies, and a sum over the Fano classes that was studied by 
A. Zinger [Z. Theorem 8.3 combines with the proof of Theorem 8.4 to give the integrality of $n_{A, g}$ in (9.7). The Fano case is much simpler: there is no need to consider clusters because for $J \in \mathcal{J}^{*}$, every embedded $J$-holomorphic curve $C$ with $c_{1}(A)>0$ is isolated and super-rigid for the constrained moduli space, and the contribution of degree 1 maps from nodal curves to $C$ is precisely

$$
G W(C)=\left(2 \sin \frac{t}{2}\right)^{c_{1}(A)+2 g-2} t^{-c_{1}(A)} q^{C}
$$

(see (1.13) and (1.14) in [ $\mathrm{Z}$ ). This completes the proof.

9.2. Genus zero invariants of semipositive manifolds. There is a similar structure theorem for the rational (genus zero) GW invariants of closed semipositive symplectic manifolds of dimension $\geq 6$. In this context, the appropriate GV transform has two parts:

(1) For $c_{1}(A)=0$, it is the Aspinwall-Morrison formula in the form given in equation (2) in $\mathrm{KP}$ :

$$
\sum_{\substack{A \neq 0 \\ c_{1}(A)=0}} G W_{A, 0}^{X}\left(\gamma_{1}, \ldots, \gamma_{k}\right) q^{A}=\sum_{\substack{A \neq 0 \\ c_{1}(A)=0}} n_{A, 0}^{X}\left(\gamma_{1}, \ldots, \gamma_{k}\right) \sum_{d \geq 1} d^{k-3} q^{d A}
$$

(2) For $c_{1}(A)>0$, it is 9.8 specialized to genus zero:

$$
G W_{A, 0}^{X}\left(\gamma_{1}, \ldots, \gamma_{k}\right)=n_{A, 0}^{X}\left(\gamma_{1}, \ldots, \gamma_{k}\right) .
$$

As before, the invariants $G W_{A, g}$ are zero for all classes $A$ with $c_{1}(A)<0$ (since $X$ is semipositive, there are no simple $J$-holomorphic spheres with $c_{1}(A)<0$ for $\left.J \in \mathcal{J}^{*}\right)$.

Theorem 9.2. For a closed semipositive symplectic manifold $X$ of dimension at least 6 , the coefficients (9.9) and (9.10) of the primary genus zero $G W$ series have the following integrality property:

$$
n_{A, 0}^{X}\left(\gamma_{1}, \cdots, \gamma_{k}\right) \in \mathbb{Z}
$$

for all $\gamma_{1}, \cdots, \gamma_{k} \in H^{*}(X, \mathbb{Z})$.

Proof. Again fix $\gamma, B$, and $A$ so that

$$
\iota=2 c_{1}(A)+(\operatorname{dim} X-6)+\sum\left(2-\operatorname{dim} \gamma_{j}\right)
$$

is zero. As before, assume $f$ is a multiple cover with reduced map $\varphi$, and $A_{i}, d_{i}$ are degrees of its components. If the domain of $\varphi$ has $r \geq 1$ components then its image has at least $r-1$ self-intersection points (since the domain of $f$ was connected). For $J \in \mathcal{J}^{*}$, these impose $(r-1)(\operatorname{dim} X-4)$ transversely cut conditions on simple maps, so the dimension of the moduli space containing $\varphi$ is

$$
\sum_{i=1}^{r}\left[2 c_{1}\left(A_{i}\right)+(\operatorname{dim} X-6)\right]-(r-1)(\operatorname{dim} X-4)+\sum_{j=1}^{k}\left(2-\operatorname{dim} \gamma_{j}\right) .
$$

Since $A=\sum d_{i} A_{i}$ and $c_{1}\left(A_{i}\right) \geq 0$, this is less than or equal to $\iota-2(r-1) \leq \iota=0$. But the moduli space is empty unless this is an equality, so we conclude that $r=1$ and $d_{1}=1$ whenever $c_{1}(A) \neq 0$. Thus the GW series again separates into a sum of $c_{1}(A)=0$ classes and a sum over $c_{1}(A)>0$ classes.

The Fano case (9.10) is classical: dimension counts imply that for generic $J$ the constrained moduli space consists only of simple maps, without any multiple cover. Thus the GW invariant is an integer.

For Calabi-Yau classes $A$ in $X^{2 m}$ we declare a $B$-constrained cluster to be elementary if its core $C$ is an embedded marked rational curve with normal bundle $N$ biholomorphic to $\mathcal{O}(-1) \oplus \mathcal{O}(-1) \oplus(m-$ $3) \mathcal{O}$, and the constraints $B_{i}$ are linear subspaces of fibers $N_{f\left(x_{i}\right)}$ of $N$ in general position in the sense that the only holomorphic section of $N$ which intersects $B_{i}$ for every $i=1, \cdots, k$ is the zero section. (Note that because $\iota=0$, the sum of the codimensions of $B_{i}$ in $N_{f\left(x_{i}\right)}$ is $2(m-3)=\operatorname{dim}_{\mathbb{R}} X-6$.) 
The core curve $C$ is then super-rigid (the constraints kill the kernel in the $\mathcal{O}$ directions) and, as proved in $\mathrm{KP}$, the contribution of its multiple covers to the primary $G W\left(\gamma_{1}, \ldots, \gamma_{k}\right)$ invariant is

$$
\operatorname{sign}_{B} C \cdot \sum_{d \geq 1} d^{k-3} q^{d C}
$$

where $\operatorname{sign}_{B} C= \pm 1$ is the sign of the core curve $C$ as an element of the cutdown moduli space $\overline{\mathcal{M}}_{A, 0, B}$. This sign can be explicitly calculated as the sign of the (transverse) intersection between the oriented linear subspace $B_{1} \times \ldots \times B_{k}$ and the image of the evaluation map ev $\mathrm{v}_{\mathbf{x}}: H^{0}\left(C, f^{*} N\right) \rightarrow$ $N_{f\left(x_{1}\right)} \times \ldots \times N_{f\left(x_{k}\right)}$ on the space of holomorphic sections of $f^{*} N$.

Now, with $\gamma, B$ and $E$ fixed, restrict attention to the cut-down moduli spaces $\overline{\mathcal{M}}_{A, 0, B}(X)$ for Calabi-Yau classes $A$ with energy at most $E$. For $J$ in the set $\mathcal{J}^{*}$ constructed after (9.4), we can decompose the fiber $\overline{\mathcal{M}}_{A, 0, B}^{J}$ into $B$-constrained clusters as in Proposition 2.4. For each such cluster, the proof of Theorem 7.1 shows that one can isotop $J$ to a elementary cluster of the above type; the resulting formula (7.3) then becomes

$$
G W^{E}(\mathcal{O}) \approx \operatorname{sign}_{B} C \cdot G W^{\text {elem }, E}\left(q^{C}\right),
$$

where the righthand side is the contribution 9.11) expressed in terms of the formal power series

$$
G W^{\text {elem }}(q)=\sum_{d \geq 1} d^{k-3} q^{d} .
$$

Since the collection of series $G W^{\text {elem }}\left(q^{A}\right)$, for Calabi-Yau classes $A$, are linearly independent as in 8.2, Theorem 8.1 also extends to the context of $B$-constrained clusters to express the lefthand side of $(9.9)$ as a linear combination, with integer coefficients, of the series $G W^{\text {elem }}\left(q^{A}\right)$. Thus the coefficients on the righthand side of 9.9 are integers.

\section{ApPENDix A}

The proofs of Lemma 1.2 and Lemma 5.5 were deferred; we give the details here and in the second appendix. The proofs are applications of transversality and the Sard-Smale Theorem. While we are primarily interested in Calabi-Yau 6-manifolds, Propositions A.4 and B.6 below apply to symplectic manifolds $(X, \omega)$ with $\operatorname{dim} X \geq 6$. Lemmas 1.2 a and 5.5 are special cases of Corollary A.5 and Proposition B.6 respectively.

As in the proof of Lemma 1.5 , every $J$-holomorphic map $f: C \rightarrow X$ lifts to a map $\widetilde{f}: \widetilde{C} \rightarrow X$ from the normalization of $C$. This lift is $J$-holomorphic and has a smooth (but not necessarily connected) domain, so it suffices to work with such maps. For every integer $\ell \geq 0$, let

$$
\mathcal{M}_{\ell, \text { simple }} \rightarrow \mathcal{J}
$$

denote the universal moduli space generalizing (5.1), consisting of equivalence classes of pairs $p=(f, J)$, where $J \in \mathcal{J}=\mathcal{J}^{l}$ and $f$ is a $W^{l, r}$ simple $J$-holomorphic map whose domain $C=$ $\left(\Sigma, j, x_{1}, \ldots, x_{\ell}\right)$ is a smooth and compact (but not necessarily connected) complex curve with $\ell$ marked points. Write $\mathcal{M}_{0, \text { simple }}$ as $\mathcal{M}_{\text {simple }}$, and let

$$
\mathcal{N E} \subset \mathcal{M}_{\text {simple }}
$$

be the subset of the universal moduli space consisting of simple maps that are not embedded. Maps in $\mathcal{N} \mathcal{E}$ either (a) are not one-to-one, or (b) are not immersions. Correspondingly, consider two types of subsets of the moduli spaces A.1): 
(a) For a pair of marked points, the inverse image of the diagonal under the evaluation map

$$
\text { ev : } \mathcal{M}_{2, \text { simple }} \longrightarrow X \times X
$$

is the subset

$$
\mathrm{ev}^{-1}(\Delta) \subset \mathcal{M}_{2, \text { simple }}
$$

consisting of simple $J$-holomorphic maps $f$ with smooth marked domain but whose image has a double point $f\left(x_{1}\right)=f\left(x_{2}\right)$.

(b) For a single marked point $x_{1}$, there is a subset $\mathcal{N} I$ of $\mathcal{M}_{1 \text {, simple }}$ consisting of simple $J$-holomorphic maps $f$ that are not immersions at $x_{1}$.

As in Section 5, we will analyze these two subsets by regarding the moduli space $\mathcal{M}_{\ell, \text { simple }}$ locally as a subspace of a slice as in (5.4). To describe $\mathcal{N} I$, let $\mathcal{L}$ be the complex line bundle over the slice whose fiber at $p=(f, J)$ is the cotangent line $T_{x_{1}}^{*} C$ to the complex curve $C$ at the marked point $x_{1}$, and let ev : $\mathcal{M}_{1, \text { simple }} \rightarrow X$ be evaluation at $x_{1}$. The bundle

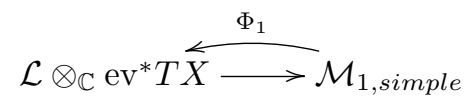

has a section $\Phi_{1}$ defined by $\Phi_{1}(f, J)=(d f)_{x_{1}}$; note that this lies in $\left(\mathcal{L} \otimes_{\mathbb{C}} e v^{*} T X\right)_{p}=T_{x_{1}}^{*} C \otimes_{\mathbb{C}} T_{f\left(x_{1}\right)} X$ because $(d f)_{x_{1}}: T_{x_{1}} C \rightarrow T_{f\left(x_{1}\right)} X$ is complex linear for $J$-holomorphic maps $f$. The zero set of $\Phi_{1}^{-1}(0)$ is the set $\mathcal{N} I$ in $(\mathrm{b})$.

Lemma A.1. For each $\ell \geq 0$, the moduli space A.1 is a $C^{k}$ separable Banach manifold for $k$ as in Proposition 5.1. Furthermore,

(a) The evaluation map A.2 is $C^{k}$ and is transverse to the diagonal.

(b) The section A.3 is $C^{k}$ and is transverse to the zero section.

Proof. Proposition 5.1 extends to show that $\mathcal{M}_{\ell, \text { simple }}$ is a separable Banach manifold, locally $C^{k}$ diffeomorphic to a $C^{l-m}$ submanifold $M_{\ell, \text { simple }}$ of a slice $\mathcal{S}$ sice $\tau_{\tau}^{m, r, l}$ for $l \geq 6, r>2,2 k \leq l-2$, and $1 \leq m \leq l-k$. Note that the section A.3 extends over the slice by the formula

$$
\Phi_{1}(f, J)=\frac{1}{2}(d f-J d f j)\left(x_{1}\right),
$$

which is equal to $d f\left(x_{1}\right)$ if $f$ is $(j, J)$-holomorphic. The Sobolev embedding theorem implies that, on the slice, the evaluation map defined by $e v(f, J)=\left(f\left(x_{1}\right), f\left(x_{2}\right)\right)$ is smooth for $m \geq 1$, and that the extension (A.4) is $C^{l-m}$ for $m \geq 2$. Consequently, the map A.2 and the section $\Phi_{1}$ in A.3 are $C^{k}$ for $k$ in the above range provided that $m \geq 2$.

Statement (a) is true by Proposition 3.4.2 of [MS]. To prove (b), we modify the proof of Lemma 3.4.3 in $\mathrm{MS}$. This involves three steps: (i) computing $d \Phi_{1}$ in certain directions, (ii) expressing the needed transversality as a differential equation with constraints on 1-jets (not just values) at the marked point, and (iii) solving this equation using weighted Sobolev spaces.

Fix a slice as above with $m \geq 2$ and a point $p=(f, J)$ on the zero set of $\Phi_{1}$, so $d f\left(x_{1}\right)=0$. Consider a variation $p_{t}=\left(f_{t}, J_{t}\right)$ of $p=p_{0}$ in $\mathcal{M}_{1 \text { simple }}$ that fixes the domain (including the complex structure and the marked point $\left.x_{1}\right)$, and also fixes the image point $f\left(x_{1}\right)$. Then $f_{t}: C \rightarrow X$ is $\left(j, J_{t}\right)$-holomorphic, and $f_{t}\left(x_{1}\right)=f\left(x_{1}\right)$ is constant. The tangent vector to $p_{t}$ at $t=0$ then has the form $(\zeta, 0, K)$, where

$$
\zeta \in W^{m, r}\left(f^{*} T X\right), \quad \zeta\left(x_{1}\right)=0, \quad K \in T_{J} \mathcal{J}^{l}, \quad \text { and } \quad \mathcal{L}_{p}(\zeta, 0, K)=0 .
$$

Here $\mathcal{L}_{p}$ is the linearization given by 4.12 and 4.13 . Calculating the first variation of

$$
\Phi_{1}\left(p_{t}\right)=\frac{1}{2}\left(d f_{t}-J_{t} d f_{t} j\right)\left(x_{1}\right) \in\left(\Lambda_{C}^{1,0} \otimes_{\mathbb{C}} f_{t}^{*} T X\right)_{x_{1}}
$$

as in [MS, Proposition 3.1.1] (with a sign change), and using the fact that $d f\left(x_{1}\right)=0$, one finds that

$$
\left(d \Phi_{1}\right)_{p}(\zeta, 0, K)=\frac{1}{2}\left(\nabla \zeta-J \nabla \zeta \circ j+\left(\nabla_{\zeta} J\right) d f j+K d f j\right)\left(x_{1}\right)=(\nabla \zeta)^{1,0}\left(x_{1}\right) .
$$


The righthand side is independent of the connection because $\zeta$ vanishes at $x_{1}$.

To prove (b), it suffices to show that A.6 is surjective, i.e. for each $\eta_{0} \in\left(\Lambda_{C}^{1,0} \otimes_{\mathbb{C}} f^{*} T X\right)_{x_{1}}$ there exists a tuple $(\zeta, 0, K)$ satisfying A.5 and such that

$$
(\nabla \zeta)^{1,0}\left(x_{1}\right)=\eta_{0}
$$

Choose a local holomorphic coordinate $z$ on $C$ centered at $x_{1}$, and write $\eta_{0}=v_{0} d z$, where $v_{0} \in\left(f^{*} T X\right)_{x_{1}}=T_{f\left(x_{1}\right)} X$. Extending $v_{0}$ to a smooth section of $T X$, pulling back by $f$, and multiplying by a bump function creates a $W^{l, r}$ section $v$ of $f^{*} T X$ supported in the coordinate chart with $v\left(x_{1}\right)=v_{0}$. We will seek a solution $(\zeta, 0, K)$ of (A.5) and (A.7) satisfying

$$
\zeta=z v+\mu
$$

where $\mu \in W^{l, r}\left(f^{*} T X\right)$ is a correction satisfying

$$
\mu\left(x_{1}\right)=(\nabla \mu)\left(x_{1}\right)=0 .
$$

This ansatz implies that $\zeta \in W^{l, r}, \zeta\left(x_{1}\right)=0$ and

$$
(\nabla \zeta)^{0,1}\left(x_{1}\right)=\left.\left(\partial z \cdot v+z(\nabla v)^{0,1}\right)\right|_{z=0}=v_{0} d z=\eta_{0} .
$$

The only remaining constraint is the last equation in A.5, which reduces to

$$
\mathcal{L}_{p}(\mu, 0, K)=D_{p}^{0} \mu+\frac{1}{2} K d f j=\alpha \quad \text { for } \quad \alpha=-D_{p}^{0}(z v) .
$$

Using the formula 4.25 for $D_{p}^{0}$, one sees that $\alpha$ is bounded. Furthermore, $|\alpha(z)|=O(|z|)$ for small $z$, as follows. As in [MS 3.1.5], we can write $D_{p}^{0}$ as the sum of a first order complex-linear operator $D_{p}^{0,1}$, and a complex anti-linear zeroth order operator $R$ given in terms of the Nijenhuis tensor of $J$ by $(R \zeta)(w)=\frac{1}{4} N_{J}(\zeta, \partial f(w))$. The calculation

$$
D_{p}^{0}(z v)=D_{p}^{0,1}(z v)+R(z v)=z D_{p}^{0,1} v+\bar{z} R v=z D_{p}^{0} v+(\bar{z}-z) R v
$$

then implies that $\alpha$ is $O(|z|)$.

Because $f$ is simple, we can now use weighted Sobolev spaces to find a solution $(\mu, K) \in$ $W^{1, r}\left(f^{*} T X\right) \oplus T_{J} \mathcal{J}^{l}$ of equation $\mathrm{A} .9$ that satisfies A.8. The appropriate weighted spaces are defined below, and the needed facts listed in Lemmas A.2 and A.3. Using the results and notation of those lemmas, the proof of (b) is completed as follows.

Fix $\delta \notin \Omega_{D}$ with $1<\delta<2$, and define $r>2$ by $\delta=2-2 / r$. Since $C$ is compact and $\alpha$ is bounded and $O(|z|)$, one sees that $\alpha$ is in $\mathcal{F}^{0, r}$, and hence is in $W^{0, r, \delta}$ by Lemma A.2 a. Because $f$ is simple, Lemma A.3. shows the existence of a solution $(\mu, 0, K) \in W^{1, r, \delta} \times\{0\} \times T_{J} \mathcal{J}^{l}$ to A.9. This $\mu$ satisfies $D_{p}(\mu, 0)=\alpha-\frac{1}{2} K d f j$ on $C^{\prime}$, and both $\alpha=-D_{p}(z v, 0)$ and $K d f j=2 \mathcal{L}_{p}(0,0, K)$ are in $\mathcal{F}^{l-1, r}$, as is seen by taking $m=l$ in $(4.26)$. But then Lemma A.2b shows that $(\mu, 0)$ lies in $\mathcal{E}^{l, r}$ and satisfies (A.8) and (A.9). This completes the proof that $\left(d \Phi_{1}\right)_{p}$ is surjective.

The weighted Sobolev spaces used at the end of the above proof are defined as follows. Fix a local holomorphic coordinate $z: U \rightarrow \mathbb{C}$ with origin at $x_{1}$, and a Riemannian metric $g_{0}$ on $C$ that is euclidean on $U$. Also fix a smooth positive function $\rho$ on $C^{\prime}=C \backslash\left\{x_{1}\right\}$ that is equal to $|z|$ on $U \backslash\left\{x_{1}\right\}$, and a constant $\delta \in \mathbb{R}$. Let $g^{\prime}$ be the metric $\rho^{-2} g_{0}$ on $C^{\prime}$. Writing $z=e^{-(t+i \theta)}$ gives coordinates $(t, \theta)$ with

$$
\rho=|z|=e^{-t}
$$

and $g^{\prime}=d t^{2}+d \theta^{2}$, so $\left(C^{\prime}, g^{\prime}\right)$ is a manifold with an end $C_{e n d}^{\prime}$ isometric to the cylinder $[0, \infty) \times S^{1}$, where $S^{1}=\mathbb{R} / 2 \pi \mathbb{Z}$. Let

$$
\mathcal{E}_{0}^{1, r, \delta}=W^{1, r, \delta}\left(f^{*} T X\right)
$$

be the completion of the set of $C^{l}$ sections of $f^{*} T X$ with compact support on $C^{\prime}$ in the norm

$$
\|\xi\|_{1, r, \delta}^{r}=\int_{C^{\prime}}\left|\rho^{-\delta} \nabla \xi\right|^{r}+\left|\rho^{-\delta} \xi\right|^{r} \quad \operatorname{dvol}_{g^{\prime}}
$$


using the norm and connection on the bundle $f^{*} T X$ induced by the metric $g^{\prime}$ on $C^{\prime}$ and the metric on $X$. The spaces $\mathcal{E}_{0}^{0, r, \delta}$ and $\mathcal{F}^{0, r, \delta}$ are defined similarly (cf. 4.20), also using the metrics $g^{\prime}$ on $C^{\prime}$ and the metric on $X$.

The following lemma gives ways to translate between these weighted spaces, which are defined using the metric $g^{\prime}$ and its Levi-Civita connection $\nabla^{\prime}$ on $C^{\prime}$, and the unweighted spaces $\mathcal{E}^{m, r}$ and $\mathcal{F}^{m, r}$, which were defined in Section 4.2 using the metric $g_{0}$ and connection $\nabla$ on $C$. Part (b) is a regularity result for the operator $D_{p}^{0}$ defined by 4.25 .

Lemma A.2. Fix $p=(f, J)$, where $f \in W^{l, r}$ is $J$-holomorphic, $J \in \mathcal{J}^{l}, l \geq 6$, and $r>2$. Then

(a) If $\alpha \in \mathcal{F}^{0, r}$ is a 1-form with $|\alpha(z)|=O(|z|)$, then $\alpha \in \mathcal{F}^{0, r, \delta}$ for all $\delta \leq 2-2 / r$.

(b) If $\mu \in \mathcal{E}_{0}^{1, r, \delta}, \delta>1$, is a weak solution of $D_{p}^{0} \mu=\alpha$ on $C \backslash\left\{x_{1}\right\}$ with $\alpha \in \mathcal{F}^{l-1, r}$, then $\mu$ extends to a solution on $C$ with $(\mu, 0) \in \mathcal{E}^{l, r}$, and with $\mu(0)=(\nabla \mu)(0)=0$.

Proof. (a) For a 1-form $\alpha$, the norms with respect to the metrics $g_{0}$ and $g^{\prime}=\rho^{-2} g_{0}$ are related by $|\alpha|_{g^{\prime}}=\rho|\alpha|_{g_{0}}$, while $d v o l_{g^{\prime}}=\rho^{-2}$ dvol $_{g_{0}}$. Hence

$$
\int_{C^{\prime}}\left|\rho^{-\delta} \alpha\right|_{g^{\prime}}^{r} d \operatorname{vol}_{g^{\prime}}=\int_{C^{\prime}}\left|\rho^{1-\delta-2 / r} \alpha\right|_{g_{0}}^{r} d \operatorname{dvol}_{g_{0}} .
$$

The righthand integral is finite for $\delta \leq 2-2 / r$ because $\alpha \in \mathcal{F}^{0, r}$ and, by assumption, $|\alpha|_{g_{0}} \leq c_{1} \rho=$ $c_{1} e^{-t}$ on $C_{\text {end }}^{\prime}$.

(b) The pointwise norm of a section of $f^{*} T X$ does not depend on the metric on the domain. Integrating $|\mu|^{r} d v^{\prime} l_{g_{0}}=\rho^{2+\delta r}\left|\rho^{-\delta} \mu\right|^{r} d v o l_{g^{\prime}}$, and again noting that $\rho$ is bounded and is equal to $|z|$ on the end, shows that $\mu \in W^{0, r}$ and that, for small $\varepsilon$, the integral over the disk $B(\varepsilon)$ centered at $x_{1}$ satisfies

$$
\int_{B(\varepsilon)}|\mu|^{r} d v o l_{g_{0}} \leq \varepsilon^{2+\delta r}\|\mu\|_{0, r, \delta}^{r} \leq c_{2} \varepsilon^{2+\delta r} .
$$

Hölder's inequality then shows that for any $s \leq r, \mu \in W^{0, s}$ and there is a constant $c_{3}=c_{3}(s)$ such that

$$
\int_{B(\varepsilon)}|\mu|^{s} d v l_{g_{0}} \leq c_{3} \varepsilon^{2+\delta s} .
$$

To apply elliptic regularity, we first verify that $D_{p}^{0} \mu=\alpha$ weakly on all of $C$. Choose a smooth 1-parameter family of cutoff functions $\left\{\gamma_{\varepsilon}\right\}$ supported on $B(\varepsilon)$ with $0 \leq \gamma_{\varepsilon} \leq 1$ and $\left|d \gamma_{\varepsilon}\right|<4 / \varepsilon$. Given any $\eta \in \mathcal{F}^{l, r}$, write $\eta=\gamma_{\varepsilon} \eta+\left(1-\gamma_{\varepsilon}\right) \eta$ and integrate:

$$
\int_{C}\left\langle\left(D_{p}^{0}\right)^{*} \eta, \mu\right\rangle-\langle\eta, \alpha\rangle=\int_{B(\varepsilon)}\left\langle\left(D_{p}^{0}\right)^{*}\left(\gamma_{\varepsilon} \eta\right), \mu\right\rangle-\int_{B(\varepsilon)}\left\langle\gamma_{\varepsilon} \eta, \alpha\right\rangle+\int_{C}\left\langle\left(1-\gamma_{\varepsilon}\right) \eta, D_{p}^{0} \mu-\alpha\right\rangle .
$$

The last integral vanishes because $D_{p}^{0} \mu=\alpha$ weakly on $C \backslash\left\{x_{1}\right\}$. Noting that $\eta$ is bounded by the Sobolev Embedding Theorem, Hölder's inequality shows that the absolute value of the middle integral on the righthand side is bounded by $\|\eta\|_{\infty}\|\alpha\|_{0, r}\left\|\gamma_{\varepsilon}\right\|_{0, s ; B(\varepsilon)} \leq c_{4} \varepsilon^{2 / s}$, where $\frac{1}{s}=1-\frac{1}{r}$. For $\varepsilon \leq 1$, the first integral on the right is similarly bounded by

$$
\int_{B(\varepsilon)}\left(\left|d \gamma_{\varepsilon}\right||\eta|+\gamma_{\varepsilon}\left|\left(D_{p}^{0}\right)^{*} \eta\right|\right)|\mu| \leq\left(\left\|d \gamma_{\varepsilon}\right\|_{0, r ; B(\varepsilon)}\|\eta\|_{\infty}+\left\|\left(D_{p}^{0}\right)^{*} \eta\right\|_{0, r}\right)\|\mu\|_{0, s ; B(\varepsilon)} \leq c_{5} \varepsilon^{1+\delta},
$$

where this last inequality follows from A.12 because $\left\|d \gamma_{\varepsilon}\right\|_{0, r ; B(\varepsilon)} \leq c_{6} \varepsilon^{2 / r-1}$ and $\left\|\left(D_{p}^{0}\right)^{*} \eta\right\|_{0, r} \leq$ $c_{7}\|\eta\|_{1, r}$ as in [MS, Proposition 3.1.11]. These bounds hold for all small $\varepsilon>0$, so the lefthand side of A.13 is equal to 0 . Thus $D_{p} \mu=\alpha$ weakly on $C$.

Elliptic regularity, as in Theorem C.2.3 of [MS], then shows that $\mu \in W^{l, r}$ and $D_{p}^{0} \mu=\alpha$ on $C$. In particular, $\mu$ is $C^{2}$ by the Sobolev Embedding Theorem. With this, A.12 and the hypothesis that $\delta>1$ imply that $\mu(0)=(\nabla \mu)(0)=0$. 
Lemma A.3. Fix $p=(f, J)$ as in Lemma A.2. Then there is a discrete set $\Omega_{D} \subset \mathbb{R}$ such that for each $\delta \notin \Omega_{D}$,

(a) The operator $D_{p}^{0}$ defined by 4.25 on $C^{l}$ sections with compact support in $C \backslash\left\{x_{1}\right\}$ extends to a Fredholm operator

$$
D_{p}^{0}: \mathcal{E}_{0}^{1, r, \delta} \rightarrow \mathcal{F}^{0, r, \delta}
$$

(b) If $d f\left(x_{1}\right)=0$ and $\delta<2$, then the operator $\mathcal{L}_{p}$ defined by 4.12) and (4.13), restricted to the subspace defined by $k=0$, induces a bounded operator

$$
\mathcal{L}_{p}: W^{1, r, \delta}\left(f^{*} T X\right) \oplus\{0\} \oplus T_{J} \mathcal{J}^{m} \rightarrow \mathcal{F}^{0, r, \delta} .
$$

(c) If, in addition, $f$ is simple, then A.15 is surjective.

Proof. (a) The Fredholm properties of the operator A.14 are determined by its asymptotic behavior in a neighborhood of $x_{1}$, which depends on the geometry of $X$ near $f\left(x_{1}\right)$. Choose a local trivialization of the complex vector bundle $(T X, J)$ in a neighborhood $V$ of $f\left(x_{1}\right)$. Our assumptions imply that $f$ is of class $C^{l}$ so, after shrinking $C_{\text {end }}^{\prime}$, we can assume that $f\left(C_{\text {end }}^{\prime}\right)$ lies in $V$. Pulling back yields a $C^{l-1}$ trivialization $f^{*} T X \cong C_{\text {end }}^{\prime} \times \mathbb{R}^{2 N}$ of $f^{*} T X$ over the end $C_{\text {end }}^{\prime}$ in which $J$ corresponds to the standard complex structure $J_{0}$ on $\mathbb{C}^{N}=\mathbb{R}^{2 N}$. This, together with the section $d \bar{z}$ of $\Lambda_{C}^{0,1}$, gives a similar trivialization of $\Lambda_{C}^{0,1} \otimes_{\mathbb{C}} f^{*} T X$ on the end.

Referring to formula 4.25 and noting that $f$ is $J$-holomorphic, we can write $D_{p}^{0} \zeta$ in these trivializations as

$$
D_{p}^{0} \zeta=\bar{\partial}_{0} \zeta+S \cdot \zeta d \bar{z}
$$

where $\bar{\partial}_{0} \zeta=\frac{1}{2}\left(d \zeta+J_{0} d \zeta j\right)$ and $S$ is a matrix-valued function depending on the pullbacks of $J, \nabla J$, and the connection form of $\nabla$ in the trivialization. The specific formula for $S$ shows that it is at least $C^{l-1}$.

After converting to $(t, \theta)$ coordinates on $C_{\text {end }}^{\prime}$ and substituting $d \bar{z}=-\bar{z}(d t-i d \theta)$, A.16 becomes

$$
D_{p}^{0} \zeta=\bar{\partial}_{0} \zeta+T \zeta
$$

where $\bar{\partial}_{0} \zeta=\frac{1}{2}\left(\partial_{t} \zeta+J_{0} \partial_{\theta} \zeta\right)$ and $T=-e^{-t+i \theta} S$. Since $S$ is bounded on $C$, we have $|T| \leq c_{1} e^{-t}$. Thus $D_{p}^{0}$ is a first order elliptic operator with $C^{l-1}$ coefficients that, in some trivialization on the end of $C^{\prime}$, is the sum of the translation invariant operator $\bar{\partial}_{0}$ and a $0^{\text {th }}$ order term $T$ that decays to 0 uniformly as $t \rightarrow \infty$. A theorem of Lockhart and McOwen LM, Theorem 6.2] then implies that A.14 is bounded and Fredholm for all $\delta$ not in a discrete set $\Omega_{D}$ (the proof assumes that $D_{p}$ has smooth coefficients, but applies without change for coefficients that are $C^{2}$ or better).

(b) Using (a), it suffices to bound the last term in (4.12). Because $f$ is $C^{2}$, the assumption that $d f\left(x_{1}\right)=0$ implies that $|d f(z)|_{g_{0}} \leq c_{2}|z|=c_{2} \rho$ on the end, and hence by compactness there is a constant $c_{3}$ such that $|d f|_{g^{\prime}} \leq c_{3} \rho^{2}$ on all of $C^{\prime}$. We then have:

$$
\|K d f j\|_{0, r, \delta} \leq\|K\|_{C^{0}}\left(\int_{C^{\prime}}\left|\rho^{-\delta} \cdot c_{2} \rho^{2}\right|^{r} d v o l_{g^{\prime}}\right)^{1 / r} \leq c_{4}\|K\|_{C^{l}},
$$

where the last inequality holds because $\rho=e^{-t}$ on the end of $C^{\prime}$ and $\delta<2$.

(c) Following the argument used to prove Proposition 5.1 if $\mathcal{L}_{p}$ is not surjective, then there exists an element $c$ of the dual space $\left(\mathcal{F}^{0, r, \delta}\right)^{*}=\mathcal{F}^{0, s,-\delta}, s=\frac{r}{r-1}>1$, such that $\left(D_{p}^{0}\right)^{*} c=0$ and 5.2 holds for every $K$ in $T_{J} \mathcal{J}^{l}$. The proof of Lemma 4.1, applied on $C^{\prime}$, shows that there is an injective point $x \in C^{\prime}$ such that $c(x) \neq 0$. Then $K=\beta_{\varepsilon} K_{0}$, as defined after 5.3 , has compact support in a neighborhood of this point $x \in C^{\prime}$ and lies in $T_{J} \mathcal{J}^{l}$, giving a contradiction as $\varepsilon \rightarrow 0$. Thus A.15 is surjective. 
Next, note that for every $\ell \geq 0$ the map

$$
\pi_{\ell}: \mathcal{M}_{\ell, \text { simple }} \rightarrow \mathcal{M}_{\text {simple }}
$$

that forgets the marked points is a submersion with index $\pi_{\ell}=2 \ell$.

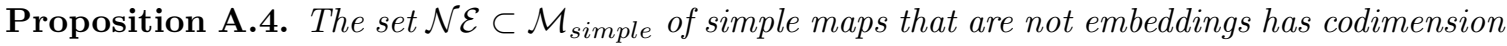
$\operatorname{dim} X-4$ in the sense of Definition 5.4 .

Proof. As above, $\mathcal{N E}$ is the union of $\pi_{1}\left(\iota_{1} S^{1}\right)$ and $\pi_{2}\left(\iota_{2} S^{2}\right)$, where $\iota_{1}: S^{1}=\Phi_{1}^{-1}(0) \hookrightarrow \mathcal{M}_{1, \text { simple }}$ and

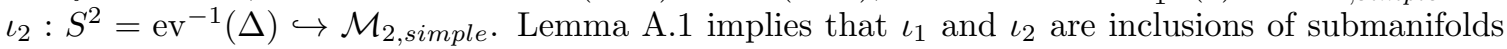
with index $\iota_{\ell}=-\operatorname{codim} S^{\ell}$ for $\ell=1,2$. One sees from A.2 and A.3 that $S^{1}$ and $S^{2}$ both have codimension $\operatorname{dim} X$. Hence $\mathcal{N} \mathcal{E}$ is a set of codimension $k$ where $k=-\operatorname{index}\left(\pi_{\ell} \circ \iota_{\ell}\right)=$ $-\operatorname{index} \pi_{\ell}-\operatorname{index} \iota_{\ell}=-2 \ell+\operatorname{dim} X \geq \operatorname{dim} X-4$.

Corollary A.5. If $X$ is a symplectic Calabi-Yau 6-manifold, there is a Baire set $\mathcal{J}^{*} \subset \mathcal{J}^{l}, l \geq 6$ or $l=\infty$, so that for each $J \in \mathcal{J}^{*}$ all simple $J$-holomorphic maps are regular, and are embeddings with pairwise disjoint images.

Proof. For $\mathcal{J}=\mathcal{J}^{l}$, the Sard-Smale Theorem implies that the regular values of the projection $\pi: \mathcal{M}_{\text {simple }} \rightarrow \mathcal{J}$ are a Baire set $\mathcal{J}_{1}$ in $\mathcal{J}$. When $X$ is a Calabi-Yau 6-manifold, the index of $\pi$ is 0 , and Proposition A.4 shows that $\mathcal{N} \mathcal{E}$ is a codimension 2 subset of $\mathcal{M}_{\text {simple }}$. Applying Sard-Smale again, there is a Baire set $\mathcal{J}_{2}$ of $\mathcal{J}$ such that for each $J \in \mathcal{J}^{*}=\mathcal{J}_{1} \cap \mathcal{J}_{2}, J$ is a regular value of $\pi$ and $\pi^{-1}(J)$ is disjoint from $\mathcal{N} \mathcal{E}$, which means that all simple $J$-holomorphic curves are embedded.

This proof extends to the space of smooth maps over $\mathcal{J}=\mathcal{J}^{\infty}$ by applying Taubes' argument, as in the proof Theorem 3.1.6(II) in [MS].

\section{Appendix B}

This second appendix is devoted to the proof of the following result, which immediately implies Lemma 5.5, and also generalizes parts (b) and (c) of Proposition 5.3. As in Appendix A, our moduli spaces and operators $D_{p}$ are defined on the Sobolev completions introduced in Section 4.2, but for notational simplicity we omit the superscripts indicating the Sobolev norms.

Proposition B.6. Suppose $\operatorname{dim} X \geq 6$ and $\mathcal{N}$ is a component of $\mathcal{M}_{\text {simple }}$ such that the projection $\pi: \mathcal{N} \rightarrow \mathcal{J}$ has index 0 . Then

(a) $\mathcal{W}^{1} \cap \mathcal{N}$ is a codimension 1 submanifold of $\mathcal{N}$, and

(b) $\left(\mathcal{W} \backslash \mathcal{W}^{1}\right) \cap \mathcal{N}$ is a subset of $\mathcal{N}$ of codimension $\geq 3$.

Furthermore, (a) and (b) hold with $\mathcal{N}$ replaced by $\mathcal{N} \cap \mathcal{M}_{C}$.

The proof is based on another construction involving the spaces (A.1) and the projections A.18. Below, we will locally regard $\mathcal{M}_{\ell, \text { simple }}$ as a subset of a slice, and write its elements as pairs $q=(p, \mathbf{x})$, where $p=\pi_{\ell}(q)=(f, J) \in \mathcal{M}_{\text {simple }}$ and $\mathbf{x}=\left(x_{1}, \ldots, x_{\ell}\right)$ are the marked points on the domain $C$ of $f$. For each $\ell \geq 0$, let

$$
\mathcal{M}_{\ell, \text { simple }}^{*} \subseteq \mathcal{M}_{\ell, \text { simple }}
$$

be the open set of all $q$ such that each of the marked points $x_{1}, \ldots, x_{\ell}$ is an injective point of $f$. Pull back the bundles $\mathcal{E}$ and $\mathcal{F}$ to $\mathcal{M}_{\ell, \text { simple }}^{*}$ by the map between slices corresponding to A.18. Let $\mathcal{E}^{\ell} \rightarrow \mathcal{M}_{\ell, \text { simple }}^{*}$ be the subbundle of $\pi_{\ell}^{*} \mathcal{E}$ whose fiber at $q=(p, \mathbf{x})$ is the set

$$
\mathcal{E}_{q}^{\ell}=\left\{\xi \in \pi_{\ell}^{*} \mathcal{E}_{p} \mid \xi^{N}\left(x_{i}\right)=0 \text { for } i=1, \ldots, \ell\right\}
$$


of elements of $\pi_{\ell}^{*} \mathcal{E}_{p}$ whose normal component vanishes at $x_{1}, \ldots, x_{\ell}$. For each $q=(p, \mathbf{x}) \in \mathcal{M}_{\ell, \text { simple }}^{*}$, $\mathcal{E}_{q}^{\ell}$ is a linear subspace of $\pi_{\ell}^{*} \mathcal{E}_{p}$ of codimension $\ell(\operatorname{dim} X-2)$, and the linearization $D_{p}$ at $p$, given in 4.13 , restricts to a linear map

$$
D_{q}^{\ell}: \mathcal{E}_{q}^{\ell} \rightarrow \pi_{\ell}^{*} \mathcal{F}_{p}
$$

Regarding $D_{q}^{\ell}$ as the composition of the inclusion $\mathcal{E}_{q}^{\ell} \hookrightarrow \pi_{\ell}^{*} \mathcal{E}_{p}$ with $D_{p}$, one sees that $D_{q}^{\ell}$ is Fredholm with index

$$
\iota_{\ell}=\operatorname{index} D_{q}^{\ell}=\operatorname{index} D_{p}-\ell(\operatorname{dim} X-2)
$$

for all $q=(p, \mathbf{x}) \in \mathcal{M}_{\ell, \text { simple. }}^{*}$. As in (5.7), let Fred ${ }_{\iota_{\ell}} \rightarrow \mathcal{M}_{\ell, \text { simple }}^{*}$ be the fiber bundle whose fiber at $q=(p, \mathbf{x})$ is the space of index $\iota_{\ell}$ Fredholm operators from $\mathcal{E}_{q}^{\ell}$ to $\pi_{\ell}^{*} \mathcal{F}_{p}$. This is stratified by submanifolds Fred $\iota_{\ell \ell}^{s}$, and

defines a section of this bundle. Let

$$
\Psi^{\ell}(q)=D_{q}^{\ell}
$$

$$
V^{\ell} \subseteq \mathcal{M}_{\ell, \text { simple }}^{*}
$$

be the open set of all $q=\left(p, x_{1}, \ldots, x_{\ell}\right)$ such that there exists an injective point $y$, distinct from the set $\left\{x_{i}\right\}$, such that $\operatorname{ker} D_{(q, y)}^{\ell+1}=0$.

Lemma B.7. The section $\Psi^{\ell}$ is transverse to Fred $_{\iota_{\ell}}^{s}$ along $V^{\ell}$, as is its restriction to $\pi^{-1}\left(\mathcal{M}_{C}\right)$. Hence for $s \geq 1$ the sets

$$
S^{\ell, s}=V^{\ell} \cap\left(\Psi^{\ell}\right)^{-1} \operatorname{Fred}_{\iota_{\ell}}^{s}=\left\{q \in V^{\ell} \mid \operatorname{dim} \operatorname{ker} D_{q}^{\ell}=s\right\}
$$

and $S_{C}^{\ell, s}=S^{\ell, s} \cap \pi_{\ell}^{-1}\left(\mathcal{M}_{C}\right)$ are submanifolds of codimension $s\left(s-\iota_{\ell}\right)$, where $\iota_{\ell}$ is given by (B.20).

Proof. To prove transversality at $q \in S^{\ell, s}$ we must show that the image of $\left(d \Psi^{\ell}\right)_{q}$ projects surjectively onto the normal space $\operatorname{Hom}\left(\operatorname{ker} D_{q}^{\ell}, \operatorname{coker} D_{q}^{\ell}\right)$ to $\operatorname{Fred}_{\iota_{\ell}}^{s}$ at $D_{q}^{\ell}$. Fix a slice containing $q$ and identify coker $D_{q}^{\ell}$ with the kernel of the adjoint operator $\left(D_{q}^{\ell}\right)^{*}$ defined as in 4.27). By contradiction, assume there exists a non-zero element of the normal space, regarded as a linear map $A_{q}: \operatorname{ker} D_{q}^{\ell} \rightarrow \operatorname{ker}\left(D_{q}^{\ell}\right)^{*}$,

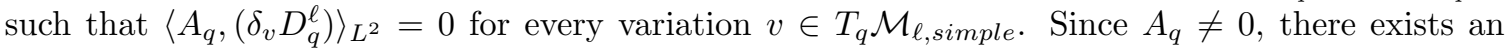
$L^{2}$-normalized $\kappa \in \operatorname{ker} D_{q}^{\ell}$ such that $c=A_{q} \kappa \in \operatorname{ker}\left(D_{q}^{\ell}\right)^{*}$ is nonzero. Fix an $L^{2}$ orthonormal basis $\left\{\kappa_{i}\right\}$ of ker $D_{q}^{\ell}$ with $\kappa_{1}=\kappa$. We then have

$$
0=\left\langle A_{q},\left(\delta_{v} D_{q}^{\ell}\right)\right\rangle_{L^{2}}=\sum_{i} \int_{C}\left\langle A_{q} \kappa_{i},\left(\delta_{v} D_{q}^{\ell}\right) \kappa_{i}\right\rangle
$$

for all $v \in T_{q} \mathcal{M}_{\ell, \text { simple }}$.

The assumption that $q \in V^{\ell}$ means, by definition, that there is an injective point $y \notin\left\{x_{1}, \ldots, x_{\ell}\right\}$ such that the map

$$
\mathrm{ev}_{x}: \operatorname{ker} D_{q}^{\ell} \rightarrow N_{f(x)} \quad \text { given by } \kappa \mapsto \kappa^{N}(x)
$$

is injective for $x=y$, and hence for all $x$ in a neighborhood of $y$. As in the proof of Lemma 4.1, there exits an injective point $x$ in that neighborhood with $c(x) \neq 0$. For this $x$, the values $\left\{\kappa_{i}^{N}(x)\right\}$ are linearly independent because $\mathrm{B} .23$ is injective. Applying Lemma B.8 below with $\xi=\kappa_{1}^{N}(x) \neq 0$ and $V=\operatorname{span}\left\{\kappa_{i}^{N}(x) \mid i \geq 2\right\}$ produces a $K$ satisfying (B.25) below.

Now proceed as in the proof of Proposition 5.3 , taking $v_{\varepsilon}=\left(0,2 \beta_{\varepsilon} K\right)$ in $(\mathrm{B} .22)$. These variations do not affect the map $f$, the complex structure on the domain or the marked points, and hence do not change the domain and range of the operators B.19). Because $D_{p}$ and $D_{q}^{\ell}$ are differential operators with the same formula, the variation $\left(\delta_{v_{\varepsilon}} D^{\ell}\right)_{q}$ is again given by (5.8) with $K$ replaced by $2 \beta_{\varepsilon} K$.

After substituting and taking the limit $\varepsilon \rightarrow 0$ as in the proof of Proposition $5.3 \mathrm{~b}$, equation (B.22) implies that 


$$
0=\sum_{i}\left\langle\left(A_{q} \kappa_{i}\right)(x),\left(\nabla_{\kappa_{i}^{N}(x)} K\right) f_{*} j\right\rangle=|c(x)|^{2},
$$

where the last equality holds because $\left(\nabla_{\kappa_{i}^{N}(x)} K\right) f_{*} j=0$ for all $i \geq 2$ by B.25. This contradicts the fact that $c(x) \neq 0$, and hence establishes the transversality of $\Psi^{\ell}$ at $q \in S^{\ell, s}$. The restriction of $\Psi$ to $\pi_{\ell}^{-1}\left(\mathcal{M}_{C}\right)$ is also transverse to Fred ${ }_{\iota \ell}^{s}$ because for each embedding $q \in S^{\ell, s}$ with image $C$, the variations $v_{\varepsilon}$ above are tangent to $\pi_{\ell}^{-1}\left(\mathcal{M}_{C}\right)$. Thus $S^{\ell, s}$ and $S_{C}^{\ell, s}$ are manifolds whose codimension, in both cases, is the dimension of the normal space to $\operatorname{Fred}_{-4 \ell}^{s}$, which is $\left(\operatorname{dim} \operatorname{ker} D_{q}^{\ell}\right)\left(\operatorname{dim} \operatorname{coker} D_{q}^{\ell}\right)=$ $s\left(s-\iota_{\ell}\right)$.

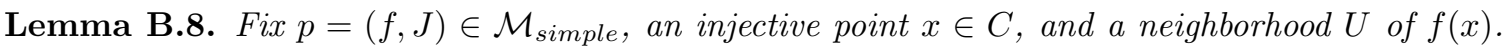
For any nonzero $\xi \in N_{f(x)}$, any subspace $V \subseteq N_{f(x)}$ not containing $\xi$, and any $c \in\left(\Lambda_{C}^{1,0} \otimes_{\mathbb{C}} f^{*} T X\right)_{x}$, there exists a $K \in T_{J} \mathcal{J}$, supported on $U$, vanishing along $f(C)$ such that, at the single point $x$ :

$$
\left(\nabla_{\xi} K\right) f_{*} j=c \quad \text { and } \quad\left(\nabla_{w} K\right) f_{*} j=0 \quad \forall w \in V .
$$

Proof. Still following the proof of Proposition $5.3 \mathrm{~b}$, there is a $K_{0} \in T_{J} \mathcal{J}$ such that $K_{0} f_{*} j=c$ at $x$. Choose a local coordinate system $\left\{z, y_{1}, y_{2}, \ldots\right\}$ centered at $f(x)$ with $z$ a local complex coordinate on $f(C)$, and $\left\{y_{i}\right\}$ real coordinates vanishing along $f(C)$, and with $\left.\frac{\partial}{\partial y_{1}}\right|_{f(x)}=\xi$ and $\left.\frac{\partial}{\partial y_{k}}\right|_{f(x)} \in V$ for $2 \leq k \leq \operatorname{dim} V+1$. Then $K=y_{1} \beta K_{0}$ has the required properties where $\beta$ is any smooth function supported in $U$ with $\beta \equiv 1$ near the origin.

Proof of Proposition B.6. We begin by making a series of observations about the images of the sets $V^{\ell}$ and $S^{\ell, s}$ under the forgetful map (A.18).

(i) The images of the $V^{\ell}$ cover $\mathcal{M}_{\text {simple }}$. If not, there would be a map $p \in \mathcal{M}_{\text {simple }}$ not in the image of any $V^{\ell}$. Choose a dense sequence $\left\{x_{1}, x_{2}, \ldots\right\}$ of distinct injective points in the domain $C$ of $p$. Then for each $\ell, q_{\ell}=\left(p, x_{1}, \ldots, x_{\ell}\right) \notin V^{\ell}$, which implies that $\operatorname{ker} D_{q_{\ell+1}+1}^{\ell+1} \neq 0$. But then $\operatorname{ker} D_{q_{\ell+1}+1} \subseteq \operatorname{ker} D_{q_{\ell}}^{\ell}$ are nontrivial nested subspaces of the finite-dimensional vector space $\operatorname{ker} D_{p}$, so have a nonzero intersection. Hence there is a nonzero $\kappa \in \operatorname{ker} D_{p}$ whose normal component vanishes at all $x_{i}$, and therefore everywhere, contradicting Lemma 4.1 .

(ii) The images of the $S^{\ell, s}$ with $s \geq 1$ cover $\mathcal{W}$. Given $p \in \mathcal{W}$, we have $\operatorname{ker} D_{p} \neq 0$. As in (i), there is a sequence $\left\{x_{i}\right\} \subset C$ and an $m>0$ such that ker $D_{q_{m}}^{m}=0$. Let $\ell$ be the largest $k$ such that $\operatorname{ker} D_{q_{k}}^{k} \neq 0$. Then by (B.21), $q_{\ell} \in S^{\ell, s}$ for $s=\operatorname{dim} \operatorname{ker} D_{q_{\ell}}^{\ell} \geq 1$, and hence $p \in \pi_{\ell}\left(S^{\ell, s}\right)$.

(iii) $S^{0,1}=\mathcal{W}^{1}$ is a submanifold of $\mathcal{M}_{\text {simple. }}$ Equation (B.21) shows that $S^{0,1} \subseteq \mathcal{W}^{1}$, while Lemma 4.1 implies $\mathcal{W}^{1} \subseteq S^{0,1}$. Hence, by Lemma B.7. $\mathcal{W}^{1}$ is a submanifold of $\mathcal{M}_{\text {simple }}$ of codimension $1-\iota_{0}$. In particular, for each component $\mathcal{N}$ of $\mathcal{M}_{\text {simple }}$ with index $D_{p}=0$, we have $\iota_{0}=0$ by B.20), so the restriction $\mathcal{W}^{1} \cap \mathcal{N}$ is a codimension 1 submanifold of $\mathcal{N}$.

(iv) $\pi_{\ell}: S^{\ell, s} \rightarrow \mathcal{M}_{\text {simple }}$ is a Fredholm map of index $2 \ell+s\left(\iota_{\ell}-s\right)$. This map is the composition of the inclusion $S^{\ell, s} \rightarrow \mathcal{M}_{\ell, \text { simple }}$, which has index $s\left(\iota_{\ell}-s\right)$ by Lemma B.7, and the map A.18) which has index $2 \ell$.

By Facts (ii) and (iii), $\mathcal{W} \backslash \mathcal{W}^{1}$ is covered by the sets $\pi_{\ell}\left(S^{\ell, s}\right)$ for $\ell \geq 0, s \geq 1$, and $(\ell, s) \neq(0,1)$. By Fact (iv) and (B.20), the intersection of each of these sets with $\mathcal{N}$ is the image of a Fredholm map of index $2 \ell-\ell s(\operatorname{dim} X-2)-s^{2} \leq 2 \ell-4 \ell s-s^{2} \leq-3$. Thus $\left(\mathcal{W} \backslash \mathcal{W}^{1}\right) \cap \mathcal{N}$ is a set of codimension 3 in the sense of Definition 5.4 .

The same proof applies if we restrict everything to $\mathcal{M}_{C}$ instead of $\mathcal{M}_{\text {simple }}$. 


\section{REFERENCES}

[BP1] J. Bryan and R. Pandharipande, BPS states of curves in Calabi-Yau 3-folds, Geometry \& Topology 5(2001), $287-318$.

[BP2] _ The local Gromov-Witten theory of curves, J. Amer. Math. Soc. 21(2008), 101-136.

[FP] K. Faber and R. Pandharipande, Hodge integrals and Gromov-Witten theory, Invent. Math. 139(2000), 173-199.

[GV] R. Gopakumar and C. Vafa, M-Theory and Topological Strings I and II, hep-th/9809187 and hep-th/9812127

[IP1] E.N. Ionel and T.H. Parker, The Gromov invariants of Ruan-Tian and Taubes, Math. Res. Lett. 4(1997), 521-532.

[IP2] _ Relative Gromov-Witten Invariants, Annals of Math. 157(2003), 45-96.

[IS1] S. Ivashkovich and V. Shevchishin, Pseudo-holomorphic curves and envelopes of meromorphy of two-spheres in $\mathbf{C P}^{2}$, arXiv:math.CV/9804014

[IS2] S. Ivashkovich and V. Shevchishin, Structure of the moduli space in a neighborhood of a cusp-curve and meromorphic hulls, Invent. Math. 136, (1999) 571-602.

[KP] A. Klemm and R. Pandharipande, Enumerative geometry of Calabi-Yau 4-folds, Commun. Math. Phys. $\mathbf{2 8 1}(2008), 621-653$.

[K] U. Koschorke, Infinite dimensional K-theory and characteristic classes of Fredholm bundle maps, in Proceedings of the Symposia in Pure Mathematics, Vol. XV, pp 95-133. AMS, Providence, R.I. 1970.

[L] J. Lee, Holomorphic 2-forms and vanishing theorems for Gromov-Witten invariants, Canad. Math. Bull. 52(2009), 87-94.

[LP1] J. Lee and T.H. Parker, A Structure Theorem for the Gromov-Witten Invariants of Kähler Surfaces. J. Diff. Geom. 77 (2007), 483-513.

[LP2] J. Lee and T.H. Parker, An obstruction bundle relating Gromov-Witten invariants of curves and Kähler surfaces, American J. of Math. 134 (2012), 453-506.

[LM] R.B. Lockhart and R.C. McOwen, Elliptic differential operators on noncompact manifolds, Ann. Scuola Norm. Sup. Pisa Cl. Sci. 12 (1985) 409-447.

[MOOP] D. Maulik, A. Oblomkov, A. Okounkov, and R. Pandharipande, Gromov-Witten/Donaldson-Thomas correspondence for toric 3-folds, Invent. Math. 186(2011), 435-479.

[MW] M. Micallef and B. White, The structure of branch points in minimal surfaces and in pseudoholomorphic curves, Ann. of Math. 141(1995), 35-85.

[MS] D. McDuff and D. Salamon, J-holomorphic curves and symplectic topology, second ed., A.M.S. Colloquium Publications, 52, AMS, Providence, RI, 2012.

[P] R. Pandharipande, Hodge integrals and degenerate contributions, Commun. Math. Phys. 208(1999), 489-506.

[PP] R. Pandharipande and A. Pixton, Gromov-Witten/Pairs correspondence for the quintic 3-fold, J. Amer. Math. Soc., 30 (2017), 389-449.

[PT] R. Pandharipande and R.P. Thomas, Curve counting via stable pairs in the derived category, Invent Math. 178(2009), 407-447.

[Pd] J. Pardon, An algebraic approach to virtual fundamental cycles on moduli spaces of pseudo-holomorphic curves, Geometry \& Topology 20 (2016), 779-1034.

[S] S. Smale, An Infinite Dimensional Version of Sard's Theorem, Amer. J. Math. 87(1965), 861-866.

[T] C. H. Taubes, Counting pseudo-holomorphic curves in dimension four, J. Diff. Geom. 44(1996), 818-893.

[Z] A. Zinger, A comparison theorem for Gromov-Witten invariants in the symplectic category, Adv. Math. 228(2011), $535-574$.

Department of Mathematics, Stanford University

E-mail address: ionel@math.stanford.edu

Department of Mathematics, Michigan State University

E-mail address: parker@math.msu.edu 